\title{
Hybrid simulations of the stellar wind interaction with close-in extrasolar planets
}

\author{
Von der Fakultät für Elektrotechnik, Informationstechnik, Physik \\ der Technischen Universität Carolo-Wilhelmina \\ zu Braunschweig \\ zur Erlangung des Grades eines \\ Doktors der Naturwissenschaften \\ (Dr.rer.nat.) \\ genehmigte \\ Dissertation
} von Erik P. G. Johansson aus Kungälv / Schweden 


\section{Bibliografische Information der Deutschen Nationalbibliothek}

Die Deutsche Nationalbibliothek verzeichnet diese Publikation in der Deutschen Nationalbibliografie; detaillierte bibliografische Daten sind im Internet über http: //dnb.d-nb. de abrufbar.

1. Referentin oder Referent: Prof. Dr. Uwe Motschmann

2. Referentin oder Referent: Prof. Dr. Karl-Heinz Glaßmeier eingereicht am: 15. Januar 2010

mündliche Prüfung (Disputation) am: 8. April 2010

ISBN 978-3-942171-35-9

uni-edition $\mathrm{GmbH} 2010$

http://www.uni-edition.de

(C) Erik P. G. Johansson

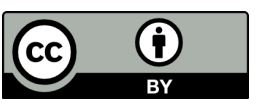

This work is distributed under a

Creative Commons Attribution 3.0 License

Printed in Germany 
Till mormor. . 



\section{Contents}

\begin{tabular}{ll}
\hline Summary & 7
\end{tabular}

\begin{tabular}{lll}
\hline & Introduction & 9
\end{tabular}

1.1 The first exoplanets . . . . . . . . . . . . . . . . . . . . . . . . . 9

1.2 Detection and observation methods . . . . . . . . . . . . . . . . 10

1.3 Close-in stellar wind interaction . . . . . . . . . . . . . . . . . 12

1.4 The purpose of this work $\ldots \ldots \ldots \ldots \ldots$

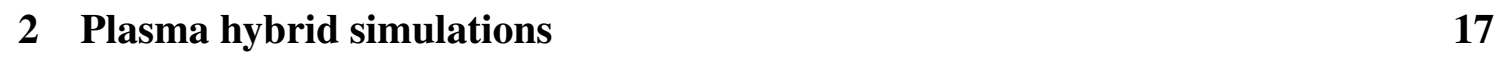

2.1 The hybrid model and its assumptions . . . . . . . . . . . . . . . . . . . 18

2.2 Some numerical considerations . . . . . . . . . . . . . . . . . . . . . . . . 20

2.3 Ionosphere, planet and boundary conditions . . . . . . . . . . . 20

2.4 Stellar wind model . . . . . . . . . . . . . . . . . 21

2.5 Coordinate system and naming conventions . . . . . . . . . . 22

3 Expanding atmospheres 25

3.1 Introduction . . . . . . . . . . . . . . . . . 25

3.2 Model parameters . . . . . . . . . . . . . . . . . . . . . . 26

3.2 .1 Stellar wind parameters . . . . . . . . . . . . . . . . . . . . . . . . . . . . 27

3.2.2 Ionospheric parameters . . . . . . . . . . . . . . . . 27

3.3 Results . . . . . . . . . . . . . . . . . . . . . . . . 29

4 Standoff distance for expanding atmospheres 39

4.1 Introduction . . . . . . . . . . . . . . . . . . . . 39

$4.2 \quad$ Analytical estimates of standoff distance $\ldots \ldots \ldots$. . . . . . . . . . . 39

4.3 Standoff distances from simulations . . . . . . . . . . . . . . . . . 40

4.4 Results . . . . . . . . . . . . . . . . . . . . . . . . 41

5 Quasiparallel stellar wind interaction $\quad 45$

5.1 Introduction . . . . . . . . . . . . . . . 45

5.2 Model parameters . . . . . . . . . . . . . . . . . . . . . . . . . . . . . . . . . . 49

$5.2 .1 \quad$ Stellar wind parameters . . . . . . . . . . . . . . . . . . . . . . . . . . . . . . . . . . . . 50

5.2 .2 Ionospheric parameters . . . . . . . . . . . . . . . . 50

5.3 Results . . . . . . . . . . . . . . . . 53

$\begin{array}{lll}6 & \text { Conclusions and outlook } & 65\end{array}$ 
\begin{tabular}{|l|l|}
\hline A Standoff distances from estimates and simulations & 69
\end{tabular}

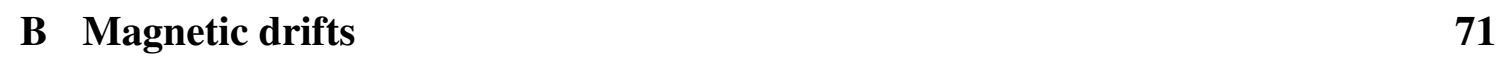

\begin{tabular}{ll}
\hline Bibliography & 73
\end{tabular}

\begin{tabular}{ll}
\hline Publications & 79
\end{tabular}

\begin{tabular}{|l|l|}
\hline Acknowledgments & 81
\end{tabular}

\begin{tabular}{lr}
\hline Curriculum Vitae & 83
\end{tabular} 


\section{Summary}

The discovery of the first planet in orbit around another Sun-like star in 1995 started a still running race to find more of these so called extrasolar planets, or exoplanets for short. One of the many remarkable discoveries that has followed is that a large fraction of exoplanets orbit their host stars on distances much smaller than any planet in the Solar System. The existence of such planets, so called close-in exoplanets, is interesting since it opens up for the possibility of qualitatively new kinds of stellar wind interaction not previously seen in the Solar System. The understanding of these interactions may be important both for future detection methods and for mass loss estimates and hence implicitly important for the evolution of exoplanetary atmospheres and for how to interpret the observed population of exoplanets.

In this work we try to investigate such close-in stellar wind interaction using primarily hybrid simulations. The hybrid simulation model describes the time evolution of plasmas in a three-dimensional box by modeling electrons as a fluid and ions as particles. A planet with a plasma producing ionosphere is then added to the simulation box and exposed to the flow of a stellar wind plasma.

Two scenarios of stellar wind interaction with unmagnetized, Earth-sized, close-in exoplanets in orbit around a Sun-like star are constructed and investigated: 1.) stellar wind interaction with an extremely hydrodynamically expanding atmosphere and therefore expanding ionosphere, and 2.) quasiparallel stellar wind interaction i.e. when the stellar wind magnetic field approaches being parallel to the stellar wind velocity in the frame of the planet. In both cases do we study three simulation runs side by side, identical in all respects except in the variation of the parameter of interest i.e. initial ionospheric radial bulk velocity and the angle between stellar wind velocity and magnetic field respectively.

We can in all simulation runs identify bow shock, magnetic draping and ion composition boundary. In the expanding ionosphere runs we can see how the expanding ionosphere pushes all these features upstream, increasing the size of the interaction region and the effective size of the obstacle. In the process it creates a significant wake behind the planet, largely void of electromagnetic fields and dominated only by the expanding ionosphere. On the dayside, little ionospheric bulk flow is actually observed in the upstream direction since dayside ions are quickly thermalized upon creation.

An attempt is also made to analytically estimate the standoff distance for magnetospheres resulting from expanding ionospheres and compare these estimates with standoff distances obtained from an extended set of expanding ionospheres simulations. Our a priori estimate only works for high standoff distances and consistently underestimates the equivalent standoff distances from simulations. The difference should partly be due to neither taking into account the difference in pressure between the upstream stellar wind 
and ionopause pressure, nor the thermalization of dayside ionospheric bulk flow. One can however attain a fairly good fit if one assumes a higher effective ionospheric production rate.

In the quasiparallel stellar wind interaction study we can observe how several generic features of quasiperpendicular interaction are modified by gradually shifting to a quasiparallel interaction. The dayside bow shock surface is replaced by a vaguely defined parallel shock that destroys the strict division between magnetosheath and stellar wind. The stellar wind also penetrates deeper into the ionosphere. We also note a strange local compression of the ionosphere that may be due to numerical error. 


\section{Introduction}

\subsection{The first exoplanets}

It was for long just assumed that just as the sky is full of stars like our Sun, the same stars should also have planets, just the way our own Sun has planets. However, observing planets around other stars, so called extrasolar planets or exoplanets for short, is not nearly as easy as just observing other stars, and therefore planets outside the Solar system had to remain only an assumption. Without tangible proof of the existence of exoplanets, our Solar System remained the one and only example of planets in the universe to the great dismay of those trying to unravel the origins of our own Solar System, and implicitly ourselves, and even more dissatisfying to those looking for, and hoping for, the possibility of life elsewhere in the universe.

A handful of exoplanet detections had indeed been reported over the years but all of them had either been retracted or never became widely accepted ${ }^{1}$ up until in 1992 when the first confirmed discovery of two planet-mass bodies beyond our Solar System was announced by Wolszczan and Frail (1992). The planets orbited the radio pulsar PSR1257 + 12 and were discovered using radio pulsar timing (see section 1.2). Although encouraging, planets around a pulsar, a swiftly rotating neutron star, were not nearly as exciting as planets around a Sun-like star would have been since they were likely to have an origin different from the Solar System planets and were surely not hospitable to life. The detection technique itself was also not useful for detecting planets around anything but pulsars.

Not many years after however, Mayor and Queloz (1995) announced that they had found an almost Jupiter-mass planet in orbit around the star 51 Pegasi using the radial velocity method (see section 1.2). The discovery was later confirmed and as is now the convention, the exoplanet was named by adding the lowercase letter " $b$ " after the name of its host star, thus giving the planet the name 51 Pegasi b (or just 51 Peg b for shor ${ }^{2}$ ). This planet too proved to be a very different creature, being about as massive as Jupiter and located at a distance of merely $0.052 \mathrm{AU}$ from its host star and therefore much closer than any celestial body in the Solar System (cf. Mercury's perihelion at $0.313 \mathrm{AU}$ ). It was

\footnotetext{
${ }^{1}$ For example, Campbell et al. $(1988)$ cautiously announced the discovery of an exoplanet around $\gamma$ Cephei using the radial velocity method, but this was not conclusively confirmed until Hatzes et al. (2003). Therefore 2003 usually counts as the discovery year of this exoplanet. Latham et al. (1989) similarly announced the discovery of either an exoplanet or a brown dwarf in orbit around HD 114762 which was later confirmed by Henry et al. (1997).

${ }^{2}$ Subsequently discovered exoplanets around the same star would be labeled using the letters $c, d$ and so on in order of discovery. The letter " $a$ " is not used to avoid confusion with the star itself. This is very similar to the naming system for binaries which use capital letters instead.
} 
in fact very unexpected that any such exoplanet would at all exist since it contradicted the theories of Solar System formation at the time, but then again, the Solar System had been the only planetary system available to build theories of planet formation on.

With the realization that exoplanet detection was feasible, more observation programs were initiated and more exoplanets were discovered. The population of known exoplanets has steadily grown bigger and more diverse ever since. Today, only one and a half decade after the discovery of 51 Pegasi b, there are about 422 known exoplanets $\mathrm{s}^{3}$.

\subsection{Detection and observation methods}

Observing exoplanets is for obvious reasons much harder than observing Solar System planets or for that matter other stars. Examining some of the detection and observation methods without making any claim of being complete should however give some insights into what information one can obtain. Some of these methods are only useful for exoplanet statistics and almost all of them are indirect. It is also more or less assumed that one has independent estimates of mass and size of the host star to be able to quantify the properties of the discovered planets. Not all methods can be applied to all exoplanets but adding together their respective strengths they form a very impressive set of detection techniques. This multitude of methods is useful not only to make it possible to detect more special cases and independently confirm detections already made but also to further constrain the observed system parameters. Every detection made based on different physical principles or variables implies additional constraints on the system. Also, most detection techniques have a bias toward massive or large close-in exoplanets since the detections are indirect and require some kind of interaction between the star and planet. Detection of exoplanets in large orbits is also harder since detection techniques generally require the planet to complete a significant part of an orbit, or even several orbits, thereby requiring very long observation programs.

The by far most productive method so far in terms of number of detections is the radial velocity (RV) method or Doppler method. It uses the fact that the orbital motion of an exoplanet implies a similarly moving host star due to the gravitational attraction between planet and star. This motion leads to a periodically changing radial stellar velocity that can be observed through its Doppler shift and has been measured down to at least $\sim 1 \mathrm{~m} / \mathrm{s}$. This way one can deduce the orbital period, semimajor axis and eccentricity. However, since the angle $i$ between the line-of-sight and the normal of the orbital plane is usually unknown, one can only obtain a minimum value for the planetary mass, $M_{\mathrm{p}} \sin i$. This is the method used by among others The High Accuracy Radial velocity Planetary Search (HARPS) project.

Radio pulsar timing takes advantage of the special property of pulsars that they emit very regular, periodic signals for which one can measure time of arrival very precisely. An orbiting planet causes the pulsar to continuously change position which in turn implies varying times of arrival for the pulsar signals. This should not be confused with the radial velocity method although it is similar.

\footnotetext{
${ }^{3}$ Retrieved on January 6th, 2010 from http://exoplanet.eu, maintained by Jean Schneider (CNRS-LUTH, Paris Observatory).
} 
The transit method makes use of the fact that a certain fraction of exoplanets will as a matter of statistics pass in front of their host stars once per orbit as seen from the Earth. Such events, so called primary transits, imply a slight attenuation ( $\sim 1 \%$ for Jupiter) of the stellar light which can then be detected. A planet passing behind the star is similarly called a secondary transit. This method obviously only works for a small percentage of exoplanets but has proven to be very productive in terms of the kinds of parameters one can determine. The exact form of the stellar light curve and spectrum during primary transit contains information about the planet's physical size, orbital velocity, atmospheric composition and atmospheric extent as well as the angle between stellar rotation axis and orbital plane etc. The spectrum and light curve during secondary transit can also give information on the spectrum from the planet itself, e.g. black-body radiation, effective temperature, albedo and reflected light (backscattering). In combination with radial velocity measurements one also obtains the planetary mass since the line-of-sight has to be parallel to the orbital plane $\left(i=90^{\circ}\right)$. Charbonneau et al. (2007) offers a good overview of the topic. It has also been suggested that high-precision transit timing, i.e. measuring tiny changes in the length and timing of recurring transits, could be used to detect exomoons (moons in orbit around exoplanets) or other non-transiting exoplanets in the same system (Dobrovolskis and Borucki 1996, Sartoretti and Schneider 1999, Holman and Murray 2005). The COnvection ROtation and planetary Transits (COROT) mission of the French National Space Agency (CNES) and the European Space Agency (ESA) uses the transit method to detect extrasolar planets. The recently launched Kepler mission of the National Aeronautics and Space Administration's (NASA) also uses this method.

Gravitational microlensing can also be used for detecting exoplanets. The light from a distant star is temporarily amplified due to gravitational lensing if a more nearby star passes in front of it on the sky. One can typically not resolve the involved stars from such an event in which case it is called microlensing. The light curve from such an event however has a well defined shape which can be distorted if the nearer star happens to have an orbiting planet in the right place. From the shape of this distorted light curve one can then constrain the mass and orbit of the exoplanet. Since this method relies on accidental alignments one has to monitor the light curves of large numbers of stars and one can obviously not repeat a particular observation since a similar alignment is unlikely to reoccur. The method however remains important for obtaining exoplanet statistics and for being very sensitive to low-mass planets in a certain orbital range. Both the Optical Gravitational Lensing Experiment (OGLE) and the Microlensing Observations in Astrophysics (MOA) detect exoplanets with this method.

Astrometry, the science of precision measurements of star locations on the sky, can also be used for detecting exoplanets by observing star movements on the celestial sphere, similar to how the RV method detects star movements in the radial direction. Since this method detects the motion in two dimensions rather than one, one can use it to deduce the orientation of the orbital plane and the planetary mass as opposed with the RV method. It is also better suited for large orbits than the RV method since it measures changes in star position, which increase with larger orbits, rather than changes in star velocity, which decrease. So far no exoplanet has been conclusively detected with this method despite many attempts. Both ESA's upcoming Gaia mission and NASA's upcoming SIM Lite Mission (formerly the Space Interferometry Mission) will use this method to detect exoplanets. 


\section{Planet Detection Methods}

Michael Perryman, Rep. Prog. Phys., 2000, 63, 1209 (updated 3 October 2007)

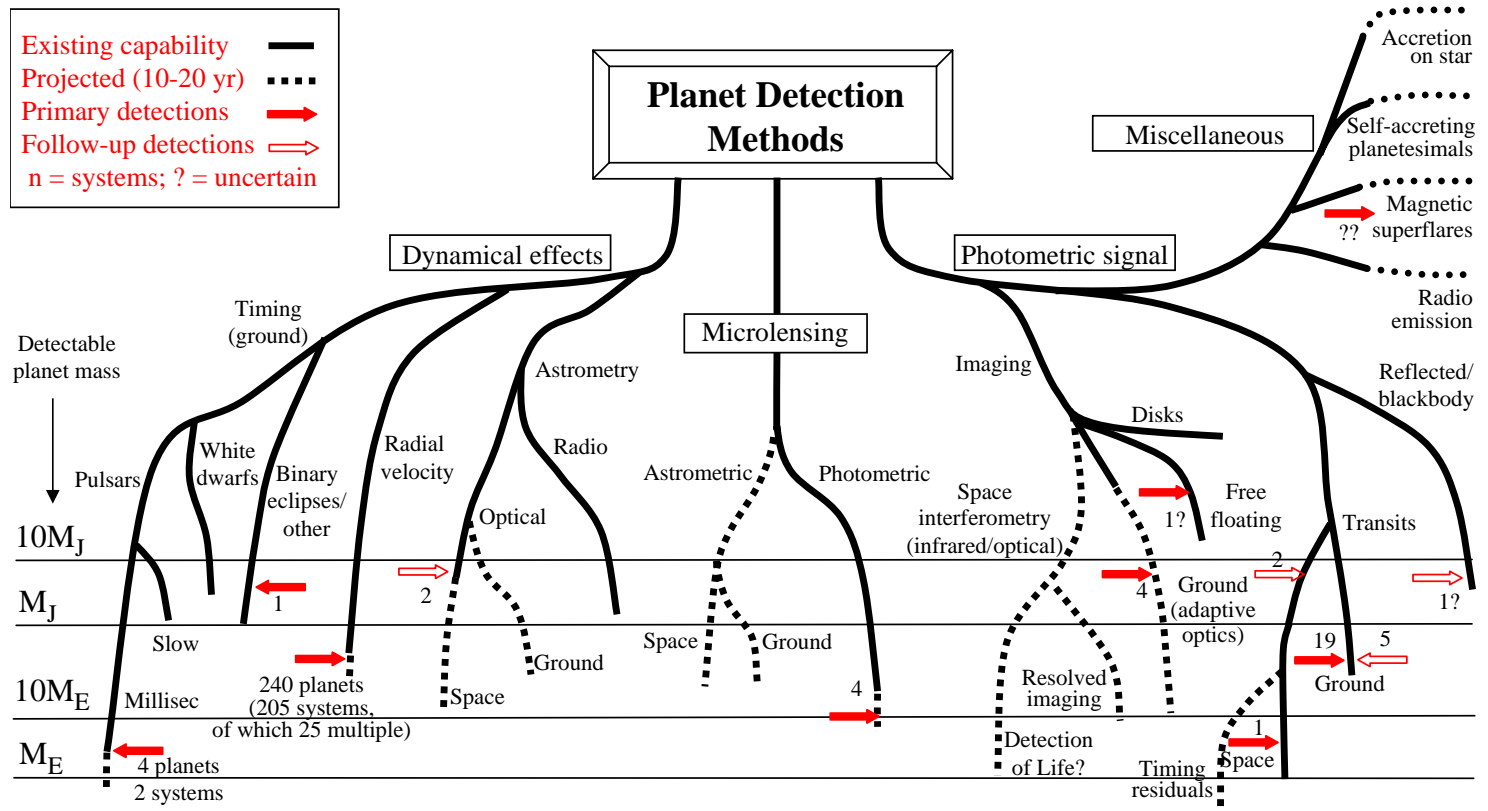

Figure 1.1: Overview of used and anticipated future exoplanet detection methods, their respective approximate minimum mass sensitivities and how many detections they have produced so far (Perryman|2000).

Direct imaging can also be used although so far with great difficulty since exoplanets are very faint. It can be used for very young (and therefore hot and infrared-emitting) exoplanets that are simultaneously large and widely separated from their host stars.

There are of course still more tricks for detecting and characterizing planets and several ones which are still only being explored. Two such methods are mentioned in section 1.3 . The Perryman diagram in Fig. 1.1 offers a popular overview over different detection techniques and their relationships. It also shows their approximate present and future detection limits for planetary mass.

\subsection{Close-in stellar wind interaction}

The diversity of newly discovered exoplanets and their orbits as well as the diversity of the type and age of their host stars opens up for new types of interaction between exoplanet and stellar wind, types of interaction which have never before had a reason to be studied. This is true in particular for close-in exoplanets, i.e. exoplanets very close to their host stars 4 . Not only do we know that such close-in exoplanets exist, but also that they are

\footnotetext{
${ }^{4}$ In this work we have, partly due to computational constraints, stretched the usual meaning of close-in to include orbital distances of $\sim 0.2$ AU whereas one normally restricts oneself to $r \lesssim 0.05-0.1 \mathrm{AU}$.
} 


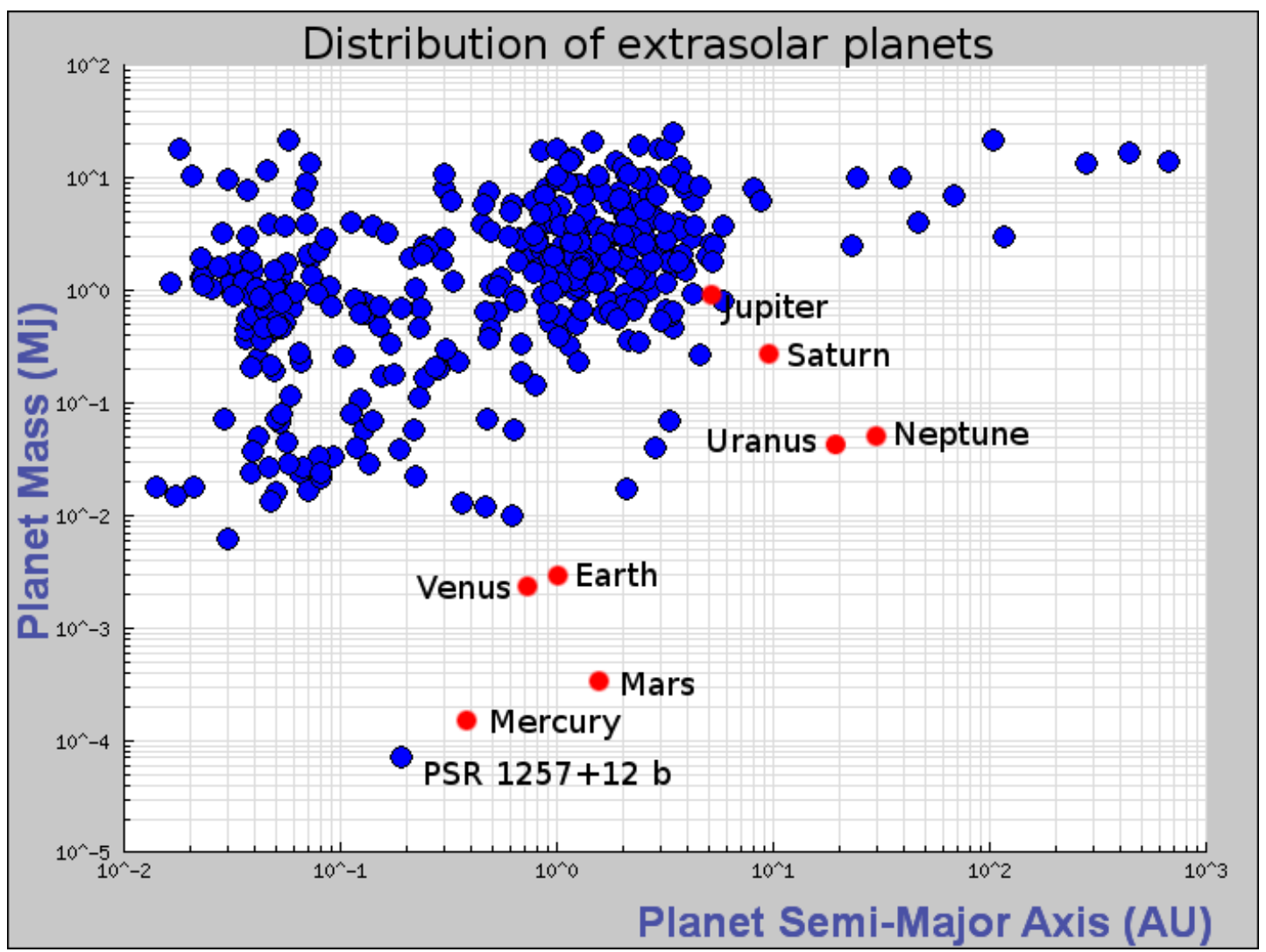

Figure 1.2: Correlation diagram between semi-major axis and mass for the known exoplanets (modified from http://www.exoplanet.eu downloaded on January 6th, 2010) with the Solar System planets added to it. Note that it does not take the type of the host star, in particular not the stellar mass, into account. It also uses the minimum mass for exoplanets detected with the radial velocity method alone.

quite common as one can see in Fig. 1.2. For example, $27 \%$ of all known exoplanets have a semimajor axis of less than $0.1 \mathrm{AU}$ and $37 \%$ have one less than $0.313 \mathrm{AU}$, i.e. closer than Mercury at perihelion (http://exoplanet.eu, January 6th 2010). It should be noted again that detection methods generally favor exoplanets in low orbits, i.e. there is some detection bias driving up these quoted percentages. Under all circumstances, these percentages still correspond to large absolute numbers of more than 100 already known exoplanets. We can find several factors and phenomena which, depending on the exact case, are relevant for the stellar wind interaction with close-in exoplanets:

1.) Close-in orbits imply greater photoionization rates and thus stronger ionospheres which, at least in the case of absent intrinsic magnetic fields, are free to react with the stronger close-in stellar winds.

2.) Stronger heating of the planetary atmospheres may in some cases lead to hydrodynamically expanding atmospheres, a type of atmosphere which extends to altitudes on the order of planetary radii and where the upper layers continuously expand to higher altitudes and are subsequently lost into space (see e.g. Watson et al. 1981, Kasting and Pollack 1983, Chamberlain and Hunten 1987, Lammer et al. 2008). This in turn implies 
similarly expanded ionospheres with ionized particles being created with an initial upward bulk motion, both qualitatively new features that can influence the stellar wind interaction (see section 3.1).

3.) As the stellar wind moves away from a host star it will at some point transition from subsonic to supersonic velocity. Thus, close-in exoplanets may in many cases be in low enough orbits to be exposed to a subsonic stellar wind (Ip et al. 2004, Preusse et al. 2005 ) instead of a supersonic wind as is the case for the Solar System planets. This opens up for the possibility of information traveling upstream through the stellar wind plasma, from an exoplanet to its host star. There are reasons to believe that this has already been observed and can be used for the detection of exoplanets in the future (see e.g. Lanza 2009, Shkolnik et al. 2009) and references therein.

4.) The Parker spiral geometry of the interplanetary magnetic field (IMF) and the higher orbital velocities for close-in exoplanets lead to a range of orbits where the IMF is approximately parallel to the stellar wind velocity in the frame of the planet, leading to potentially very different types of magnetospheres since many common features of stellar wind interaction with planets depend on the IMF having a component perpendicular to the stellar wind direction. See section 5.1 .

5.) It is known that all magnetized planets in the Solar System, in particular Jupiter, emit low frequency radio waves originating from the magnetic polar regions and being powered by the solar wind (Zarka 1998). Therefore it is expected that massive, magnetized Jupiter-like close-in exoplanets, exposed to the much stronger close-in stellar winds, are strong radio emitters, possibly detectable from Earth (see e.g. Griessmeier et al. 2007a b, Lazio et al. 2004, Farrell et al. 1999, Zarka et al. 2001). This would not only be a new method of (direct) exoplanet detection but also lead to implicit measurements of e.g. intrinsic magnetic fields, stellar wind and planetary rotation. Although detection attempts have been made, none has been successful so far (e.g. Bastian et al.2000, Farrell et al.2003).

6.) Tidal interaction with the star can for sufficiently short orbital distances circularize the orbit as well as slow down or even halt a planet's rotation (Grießmeier et al. 2009). Absence of planetary rotation in turn completely or mostly eliminates any internal dynamo and therefore intrinsic magnetic field (Griessmeier et al. 2004). This leaves the atmosphere and ionosphere unprotected from interaction with the stellar wind and coronal mass ejections (CMEs) (Khodachenko et al. 2007). This implied weakening of intrinsic magnetic fields also puts a limit on the effectiveness of the abovementioned radio emission from magnetized close-in exoplanets.

It should also be mentioned that the variety of host stars alone adds greatly to the parameter space for these systems. The stellar wind as well as ionizing radiation varies significantly with age and type of star (Griessmeier et al. 2007a, Ribas et al. 2005). As mentioned, several of these factors are very relevant for future exoplanet detection and observation methods but also for the atmospheric mass loss processes and therefore implicitly the evolution of exoplanetary atmospheres and how one should interpret the observed population of exoplanets. 


\subsection{The purpose of this work}

The domination of giant planets within the population of known close-in exoplanets is in all likelihood an observational effect and therefore we speculate, based on the theory and simulations of planet formation as well as the statistics of known exoplanets (Lin 2006, Raymond et al. 2006, Lovis et al. 2006) that there is also a significant population of still undiscovered terrestrial close-in exoplanets. The primary purpose of this work is to take a look at two principal scenarios for stellar wind interaction with unmagnetized terrestrial close-in exoplanets: interaction with hydrodynamically expanding atmospheres and quasiparallel stellar wind interaction. We do this by the means of numerical plasma hybrid simulations, a plasma simulation model that represents ions as particles and electrons as a fluid. This work should be seen as both a part of the ongoing effort to model and understand the diverse population of discovered exoplanets, and as a natural continuation and extension of the plasma hybrid simulation work that has previously been carried out on the interaction of various celestial bodies in the Solar System with their particular plasma environments, in particular the solar wind. This includes among others Boesswetter et al. (2004, 2007), Roussos et al. (2008), Simon et al. (2006, 2007a b), Martinecz et al. (2009), Kallio and Janhunen (2003). 



\section{Plasma hybrid simulations}

Two similar plasma simulation codes have been used for this work. The first one was introduced in Bagdonat and Motschmann (2002) and was used for simulating solar wind interaction with comets, but was later modified to also be able to simulate interaction with other objects like planets, moons and the plume of Enceladus (see e.g. Boesswetter et al. 2004, 2007, Simon et al. 2006, Johansson et al. 2009, Kriegel et al.2009). The second and newer plasma simulation code, the "Adaptive Ion Kinetic Electron Fluid" code (AIKEF), is essentially an improved successor to the first. It is built largely on the same physical model and numerical algorithms as the first code and will be introduced in Mueller et al. (2010). Although we have largely used the same features of both codes, AIKEF still does represent an improvement in terms of speed (partly due to parallelization), ease of use, lower memory consumption, etc.

The basic task of both these codes is to numerically calculate the time evolution of one or several plasma species in a three-dimensional simulation box. The simulation box contains some sort of obstacle, e.g. a planet, which both may and may not produce plasma on its own, for example through an ionosphere. This obstacle is then immersed in some kind of plasma flow like the solar wind, or as in our more general case, a stellar wind, and time-integrated until a quasistationary state is reached and all traces of the (artificial) initial state are gone.

To represent and time integrate the plasmas we use a hybrid model in which the ions are modeled as classical particles and the electrons as an electron fluid. This model, as opposed to pure fluid approaches like magnetohydrodynamics (MHD), has the advantages of being able to handle non-Maxwellian (ion) velocity distributions and resolve kinetic effects such as gyrations when they are larger than the cell size. As we will see in section 5.1, it should also be better suited for treating quasiparallel shocks than MHD.

The following sections describe the physical assumptions and approximations needed to arrive at our physical model, the equations which the two simulation codes try to numerically solve, how we handle boundary condition plus some numerical remarks. We will neither distinguish between the two codes nor will we go into the actual numerical integration schemes used but instead refer to Bagdonat and Motschmann (2002), Mueller et al. (2010). Since all our hybrid simulations also require the input of some stellar wind parameters we will end the chapter with a section describing our model for calculating those parameters as well as a section on our coordinate system and our naming convention for cross sections. 


\subsection{The hybrid model and its assumptions}

The equivalent number of actual physical ions in a magnetosphere-sized simulation box is in practice too great to simulate by many orders of magnitude. Therefore the hybrid model uses so called superparticles or macroparticles instead. These superparticles can be understood as either representing large numbers of ions "moving together" or as randomly sampled ions which get to represent the full behavior of the plasma. Either way, these superparticles move as physical ions and are weighted accordingly when calculating the moments of the particle distribution, i.e. density, bulk velocity, etc. All field values, i.e. densities, the magnetic field and so on, are calculated on a grid of nodes throughout the simulation box. The hybrid model also assumes that the plasma is collisionless with exception of resistivity and ion-neutral drag.

The rest of this section shows which physical assumptions we need and how we use these to derive expressions for the time derivatives of the quantities that describe the state of the system (the magnetic field and the superparticle velocities and positions). It is implicit throughout the derivation that local ion densities and ion bulk flow velocities can be calculated from the set of superparticles.

We begin by neglecting the electron mass $m_{\mathrm{e}}$

$$
m_{\mathrm{e}}=0
$$

and assuming quasineutrality, i.e.

$$
n_{\mathrm{i}}=n_{\mathrm{e}}
$$

where $n_{\mathrm{i}}$ and $n_{\mathrm{e}}$ are the total ion and electron densities!

We also assume, on the one hand, that every ion species $s$ is associated with an electron pressure term $p_{\mathrm{e}, s}$ equivalent to that of an adiabatic electron fluid with a number density $n_{\mathrm{e}, s}$ which we assume is equal to the number density $n_{\mathrm{i}, s}$ for the ion species in question. The total electron pressure $p_{\mathrm{e}}$ is thus the sum of these electron pressures,

$$
p_{\mathrm{e}, s} \propto\left(n_{\mathrm{e}, s}\right)^{\kappa_{s}}, \quad p_{\mathrm{e}}=\sum_{s} p_{\mathrm{e}, s}
$$

where $\kappa_{s}$ is the adiabatic exponent. We use $\kappa_{s}=2$ instead of $5 / 3$ in this work since the electrons effectively have only two degrees of freedom because of the strong magnetic fields.

On the other hand, we assume that there is just one momentum equation for all the electron fluids together.

$$
\underbrace{\frac{\mathrm{D}}{\mathrm{D} t}\left(n_{\mathrm{e}} m_{\mathrm{e}} \boldsymbol{u}_{\mathrm{e}}\right)}_{=0}=-e n_{\mathrm{e}}\left(\boldsymbol{E}+\boldsymbol{u}_{\mathrm{e}} \times \boldsymbol{B}\right)-\nabla p_{\mathrm{e}}+e n_{\mathrm{e}} R \boldsymbol{j} .
$$

$\boldsymbol{u}_{\mathrm{e}}$ is here the bulk electron velocity, $e$ the elementary charge, $\boldsymbol{E}$ and $\boldsymbol{B}$ the electric and magnetic fields, $R$ a scalar resistivity and $j$ the total charge current. The left-hand side is zero due to setting $m_{\mathrm{e}}=0$. This contradiction of having several electron fluids when

\footnotetext{
${ }^{1}$ This equation as wells as this entire section assumes that all ion species have a charge of one although the derivations can be generalized to arbitrarily (positively) charged ions.
} 
calculating electron pressure and only one for the momentum equation is a compromise between having one and several electron fluids. A full treatment of several electron fluids would require several connected momentum equations which would make the numerical problem much harder to solve.

The last assumption before our first important partial result is Ampère's law with the Darwin approximation, i.e. $\partial_{t} \boldsymbol{E}=0$

$$
\nabla \times \boldsymbol{B}=\mu_{0} \boldsymbol{j}+\frac{1}{c^{2}} \underbrace{\frac{\partial \boldsymbol{E}}{\partial t}}_{=0}
$$

where $c$ is the speed of light and $\mu_{0}$ is the permeability of vacuum. This assumption can be shown to be true for a collisionless plasma at frequencies lower than the plasma frequency $\omega_{\text {pe }}($ Bagdonat 2005).

The assumption of quasineutrality implies that the total current can be expressed as

$$
\boldsymbol{j}=e n_{\mathrm{i}}\left(-\boldsymbol{u}_{\mathrm{e}}+\boldsymbol{u}_{\mathrm{i}}\right)
$$

where $\boldsymbol{u}_{\mathrm{i}}$ is the total ion bulk flow velocity. This is all the extra information we need to calculate the electric field as a function of magnetic field, ion density and ion velocity.

$$
\boldsymbol{E}=-\left(\boldsymbol{u}_{\mathrm{i}} \times \boldsymbol{B}\right)+\frac{\boldsymbol{j} \times \boldsymbol{B}}{e n_{\mathrm{i}}}-\frac{\nabla p_{\mathrm{e}}}{e n_{\mathrm{i}}}+R \boldsymbol{j}
$$

Using Faraday's law

$$
\nabla \times \boldsymbol{E}=-\frac{\partial \boldsymbol{B}}{\partial t}
$$

the vanishing magnetic divergence $\nabla \cdot \boldsymbol{B}=0$ and Eq. 2.7 we can calculate the time derivative of the magnetic field as a function of magnetic field, ion density, ion velocity and current which in turn is a function of the magnetic field via Eq.2.5.

$$
\frac{\partial \boldsymbol{B}}{\partial t}=\nabla \times\left(\boldsymbol{u}_{\mathrm{i}} \times \boldsymbol{B}\right)-\nabla \times\left(\frac{\boldsymbol{j} \times \boldsymbol{B}}{\rho_{\mathrm{c}}}\right)-\nabla \times(R \boldsymbol{j})
$$

The motion of each superparticle is identical to that of a physical ion and is therefore subject to the same forces i.e.

$$
\frac{\mathrm{d} \boldsymbol{v}_{\boldsymbol{p}}}{\mathrm{d} t}=\frac{q_{p}}{m_{p}}\left(\boldsymbol{E}+\boldsymbol{v}_{\boldsymbol{p}} \times \boldsymbol{B}-R \boldsymbol{j}\right)-\eta n_{\mathrm{n}}\left(\boldsymbol{v}_{\boldsymbol{p}}-\boldsymbol{u}_{\mathrm{n}}\right)-\frac{G M_{\mathrm{p}}}{r^{2}} \hat{r}
$$

where $\boldsymbol{v}_{\boldsymbol{p}}, q_{p}$ and $m_{p}$ are the velocity, charge and mass of a superparticle $p . n_{\mathrm{n}}$ and $\boldsymbol{u}_{\mathrm{n}}$ are prescribed (postulated) values for neutral atmosphere density and velocity. $\eta$ is an ion-neutral drag constant, measuring the strength of the drag force on the ions due to collisions with neutrals. $G$ is the gravitational constant, $M_{\mathrm{p}}$ is the planetary mass, $r$ is the distance from the center of the planet and $\hat{r}$ is a unit vector pointing away from the planet. We will however neglect both resistivity and ion-neutral drag in this work and therefore set $R=0$ and $\eta=0$.

The system described above is completely described by the magnetic field and the positions and velocities of the superparticles. All other quantities can be derived from these variables. Thus it is sufficient to numerically integrate Eqs. 2.9 and 2.10 to determine the time evolution of the system. 


\subsection{Some numerical considerations}

As mentioned, we will not go into the actual numerical integration scheme but a few observations should be made however. One limiting factor for particle simulations is the strength of the magnetic field, in our case typically of the order of the stellar wind magnetic field $B_{\mathrm{sw}, 0}$ implying a typical ion gyration frequency of

$$
\omega_{\mathrm{g}, \mathrm{i}}=\frac{e B_{\mathrm{sw}, 0}}{m_{\mathrm{i}}}
$$

where $m_{\mathrm{i}}$ is the mass of the ion in question. Our hybrid model models the ion trajectory as a series of straight line segments with one line segment per time step. This implies that the timescale $\omega_{\mathrm{g}, \mathrm{i}}{ }^{-1}$ effectively sets an upper limit on the time step $\Delta t$ since every gyration has to be resolved

$$
\omega_{\mathrm{g}, \mathrm{i}}^{-1} \gg \Delta t
$$

or equivalently, rearranged into the form of a constraint on how strong magnetic fields we can accurately simulate

$$
\frac{m_{\mathrm{i}}}{e \Delta t} \gg B_{\mathrm{sw}, 0} \text {. }
$$

This constraint is particularly important for this work since the stellar wind magnetic field strength increases for shorter orbital distances.

Furthermore, keeping the non-zero electron pressure in Eq. 2.3 while neglecting electron mass (Eq. 2.1) is not a completely trivial statement since

$$
p_{\mathrm{e}}=n_{\mathrm{e}} k_{\mathrm{B}} T_{\mathrm{e}} \propto m_{\mathrm{e}} v_{\mathrm{e}, \mathrm{th} .}^{2} .
$$

This electron pressure can be seen as adding a term to the electric field in Eq. 2.4 which in turn gives rise to an anomalous non-zero charge density proportional to $\nabla^{2} p_{\mathrm{e}}$ and violating the assumption of quasineutrality. If $\lambda_{\mathrm{D}}$ is the Debye length and $L$ is the typical length scale, then it can be shown that this error is small when $\lambda_{\mathrm{D}} \ll L$, which we know is satisfied.

One can also note that the divergence of the magnetic field is not explicitly set to zero in the integration. It does however follow from Faraday's law, Eq. 2.8, and therefore implicitly from Eq. 2.9 which is based upon it, that the divergence of the magnetic field will not change over time. Therefore the hybrid model simply solves the problem by using $\nabla \cdot \boldsymbol{B}=0$ as an initial condition and a boundary condition. In the case of AIKEF, we have in addition used its built-in divergence cleaning to remove any divergence that may appear from numerical errors.

Lastly, in order to avoid numerical instabilities we have to smoothen the electromagnetic fields once per time step. This is done by replacing the value of the field in every node with a weighted average of the field at the node itself and the surrounding nodes. The downside of this procedure is that it results in an artificial diffusion of the electric and magnetic fields similar to that of a non-zero resistivity $R$.

\subsection{Ionosphere, planet and boundary conditions}

The ionosphere is implemented as a region inside the simulation box where ionospheric plasma, i.e. superparticles, are injected. Therefore we normally refer to the ionosphere 
as a region where ionospheric plasma is produced and not to where that same plasma necessarily later resides. It is important in this context to understand that any notion of neutral atmosphere is never part of any simulation more than as the conceptual motivation for plasma sources and their properties, i.e. ionospheres, and possibly to motivate the presence of ion-neutral drag as described in Eq. 2.10.

The planet obstacle is, apart from its ionosphere, described as a sphere with a superparticle absorbing surface and a manually set inner density which should try to approximately match the ionospheric density just outside the surface. The reason for this is that the difference in density between outside and inside of the planet has consequences for the electric field through the electron pressure gradient in Eq. 2.7. The magnetic field is allowed to propagate through the planet, i.e. there is no conducting core although this should be of lesser importance since the conducting ionosphere is the true obstacle.

The simulation box boundaries are all inflow boundaries except the rear, downstream boundary which is an outflow boundary. The inflow boundaries removes all the superparticles in the outermost layer of cells once per time step and then immediately inserts a set of new ones corresponding to an undisturbed stellar wind. Electromagnetic field values are set to be the stellar wind background values. The outflow boundary simply removes all the superparticles that go beyond it and has the electromagnetic field gradients set to zero.

\subsection{Stellar wind model}

All our simulations require some kind of input parameters describing the stellar wind. The stellar wind which an object is exposed to depends on the location of the object, in particular on the distance to its host star. It also depends in principle on the type of star, its age and the time in the stellar cycle (cf. solar cycle). Since little is known about the stellar winds of other stars we use the more well-known present-day solar wind as a model. We will not consider time variations in the solar wind such as turbulence, sector boundaries, solar minima and maxima nor differences due to the star's age. Furthermore we will only consider a stellar wind consisting of ionized hydrogen alone. Thus, for our purposes the local unperturbed stellar wind is described by the number density $n_{\mathrm{sw}, 0}$, the velocity vector $\boldsymbol{v}_{\mathrm{sw}, 0}$, the magnetic field vector $\boldsymbol{B}_{\mathrm{sw}, 0}$ and the ion and electron temperatures $T_{\mathrm{sw}, \mathrm{i}, 0}$ and $T_{\mathrm{sw}, \mathrm{e}, 0}$.

We use the Parker model (Parker 1958) of the stellar wind to calculate $\boldsymbol{v}_{\mathrm{sw}, 0}$ for a given orbital distance. This model assumes an isothermal, spherically symmetric stellar wind that behaves as a gas (i.e. with a weak magnetic field). By fitting the model to the Earth solar wind speed of $425 \mathrm{~km} \mathrm{~s}^{-1}$ at a distance of $1 \mathrm{AU}$ (Schwenn and Marsch 1990) we obtain the stellar wind velocity as a function of distance $a$ from the star.

The stellar wind density $n_{\mathrm{sw}, 0}$ is calculated by assuming mass conservation and spherical symmetry together with the velocity profile derived above.

$$
n_{\mathrm{sw}, 0}=\frac{C}{r^{2} v_{\mathrm{sw}, 0}}
$$

where $C=6.34 \cdot 10^{34} \mathrm{~s}^{-1}$ is a constant (Mann et al. 1999). 
Stellar wind temperatures are estimated using the scaling law for electron temperature from Schwenn and Marsch (1991)

$$
T_{\mathrm{sw}, \mathrm{e}, 0}=T_{0} r^{-\gamma}
$$

where $T_{0}$ is the electron temperature at $1 \mathrm{AU}$ and $r$ is the distance to the star in astronomical units ${ }^{2}$. We use $T_{0}=10^{5} \mathrm{~K}$ and $\gamma=0.7$. The ion temperature is calculated using $T_{\mathrm{sw}, \mathrm{i}, 0}=T_{\mathrm{sw}, \mathrm{e}, 0} / 2$ (Sonett et al. 1972$)$.

There are of course also methods for calculating the strength and direction of the IMF for different orbital distances. We are however due to the computational constraint in Eq. 2.13 forced to work in a low magnetic field limit and will therefore in practice let that determine the strength of the IMF.

To complicate matters further, we are only interested in the above quantities in the frame of the planet which is different from that of the star due to orbital motion. The effective stellar wind speed is therefore somewhat higher and in a different direction. This also changes the relative angle $\alpha_{\mathrm{sw}, 0}$ between the stellar wind velocity and the magnetic field. We will not use any precisely calculated value of this angle but note that it can in principle be obtained using additional assumptions of a frozen-in magnetic field and the rotation rate of the star. See section 5.1 .

\subsection{Coordinate system and naming conventions}

We will consistently follow the convention that the stellar wind in the frame of the planet always travels in the positive $x$ direction and that the magnetic field vector always lies in the $x y$ plane with nonnegative $x$ and $y$ components. This is depicted in Fig. 2.1. Since both the stellar wind velocity and magnetic field are in the ecliptic plane in some average sense we will refer to the $x y$ plane as the equatorial plane. Although our simulated planets do not rotate and thus do not really have an equator, we still use this name since the equatorial plane of a rotating planet usually approximates the ecliptic plane. Following this logic we can now think of the positive $z$ as "north" and negative $z$ as "south" and therefore refer to the $x z$ plane as the polar plane since it intersects both the "north pole" and the "south pole". The $y z$ plane will be referred to as the terminator plane.

\footnotetext{
${ }^{2}$ It can be noted that this varying stellar wind temperature in principle contradicts the Parker solar wind model which assumes an isothermal solar wind.
} 


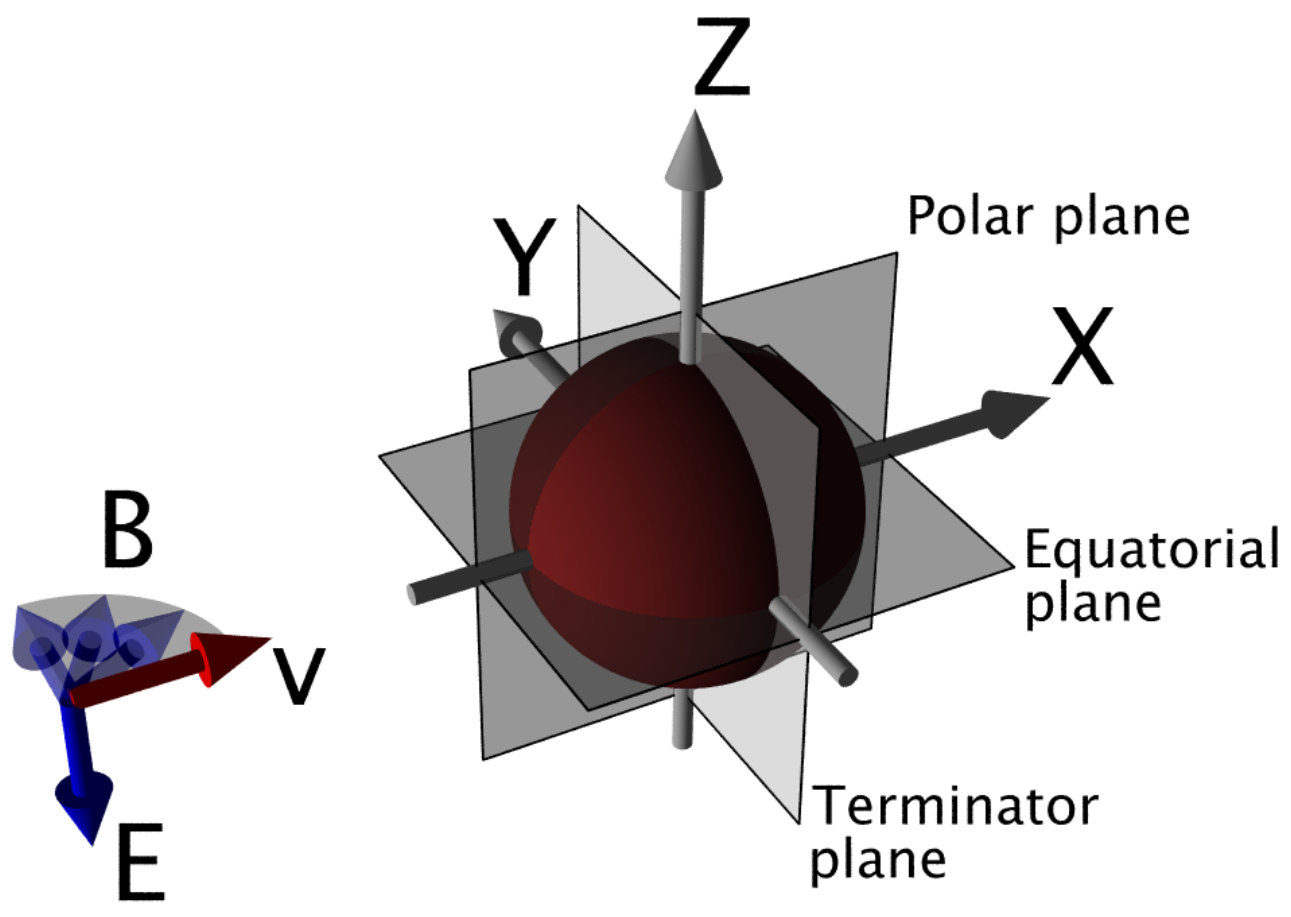

Figure 2.1: Cartoon showing the conventions for the orientation of coordinate axis and stellar wind in this work. The equatorial plane refers to the $x y$ plane, the polar plane to the $x z$ plane and the terminator plane to the $y z$ plane. $v, B$ and $E$ refer to the velocity, magnetic field and electric field of the undisturbed stellar wind, i.e. before the stellar wind interacts with planet. The stellar wind velocity $v$ is always in the positive $x$ direction. The stellar wind magnetic field $B$ always lies in the equatorial $(x y)$ plane in the quadrant with $0 \leq B_{x}$ and $0 \leq B_{y}$, although the exact magnetic field direction varies. The stellar wind electric field $E=-v \times B$ is therefore always in negative $z$ direction. 



\section{Expanding atmospheres}

\subsection{Introduction}

From the point of view of space physics we are used to thinking of an atmosphere as a largely static configuration, albeit usually with a continuous but very small loss of material through various mechanisms, so called atmospheric escape. Maybe the most well-known example of this is Jeans escape, i.e. when atoms, or possibly molecules, at the very high end of the velocity distribution escape simply by having a thermal velocity greater than the escape velocity, while at the same time being so high up in the atmosphere that they have a sufficiently high chance of not colliding with other atmospheric particles before escaping.

Other mechanisms, for example certain chemical reactions, can also provide atoms with the necessary kinetic energy to escape. Charged particles can be removed by solar wind pick-up, i.e. ions which are produced high enough in the atmosphere are accelerated away by the convective electric field of the surrounding stellar wind. There exists however a more extreme but until recent years less well-known form of atmospheric escape known as hydrodynamic escape, which is what you have in a hydrodynamically expanding atmosphere. This is something which occurs when an atmosphere is sufficiently heated and the upper layers start to expand hydrodynamically, i.e. as a collisional gas, up to altitudes on the order of planetary radii and eventually escape into space. A qualitative argument for how this can occur can be obtained by first considering the simple approximation of an atmosphere as a static ideal gas on a "flat" planet with a homogeneous gravitational field. This leads to a static solution with a density profile that decreases exponentially with altitude. However, if the gas is sufficiently heated, the scale height increases and the planet can no longer be regarded as flat, nor can the gravitational field be regards as homogeneous. One is in other words forced into using a spherically symmetric geometry with a varying gravitational field. However, there is no static solution in a spherically symmetric geometry and one is instead led to use a solution with a non-zero radial atmospheric velocity! This is basically the same argument as to why there has to be a solar wind (Parker 1958). Yet another way of looking at it is through energy balance. If an atmosphere is heated faster than it is cooled it will start to expand (have non-zero radial velocity). This in turn will allow it to cool adiabatically but only for as long as the expansion continues.

A related concept which is sometimes confused with hydrodynamic escape is blowoff which is when a lighter escaping gas is able to carry heavier constituents with it into space faster than those heavier constituents can escape themselves by Jeans escape alone

\footnotetext{
${ }^{1}$ This is not completely correct. There is a static solution, i.e. with zero radial atmospheric velocity, but it goes toward a non-zero density in the limit of high altitudes.
} 
(Chamberlain and Hunten 1987).

The subject of hydrodynamically expanding atmospheres has been investigated in the context of the ancient atmospheres of Venus, Earth and Mars (see e.g. Watson et al.1981, Kasting and Pollack 1983, Chamberlain and Hunten 1987, Lammer et al. 2008, Kulikov et al. 2007, and references therein) but it did not receive much attention until recent years with the now famous observations of the expanded atmosphere of the transiting exoplanet HD 209458 b (Vidal-Madjar et al. 2003, 2004). In brief, the planet was found to block out far more light in H I ( $15 \%$ absorption over stellar Lyman $\alpha$ emission line) during primary transit $\left.\right|^{2}$ than the body of the planet itself ( $\sim 1.5 \%$ absorption). This implied that it was surrounded by a large hydrogen atmosphere several planetary radii large thus filling up its entire Roche lobe and consequently most likely undergoing hydrodynamic escape. It was later discovered that also the absorption depths of O I and C II were significant and thus also present in the extended upper atmosphere, one of several observed indications of blowoff.

The HD 209458 b scenario of hydrodynamic expansion should in principle not be such an unusual scenario considering that HD $209458 \mathrm{~b}$ belongs to a large class of exoplanets known as close-in extrasolar giant planets (cEGP:s), or more colloquially referred to as hot Jupiters or roasters, which should be exposed to similar conditions. These are Jupiter-mass exoplanets in close-in orbits with semimajor axes of $\lesssim 0.1 \mathrm{AU}$.

As we have already mentioned in section 1.4, we speculate that there is also a population of close-in terrestrial planets yet to be discovered and that they too are exposed to the harsh conditions that could trigger hydrodynamic escape. We have therefore performed a simulation study of the magnetospheric consequences of the expanding atmosphere of a close-in terrestrial exoplanet. This study has been published in Johansson et al. (2009).

\subsection{Model parameters}

Our simulation scenario is in several ways chosen to approximate that of an Earth-like planet in a close-in orbit around a Sun-like star. Thus we choose to work with a planet with mass $M_{\mathrm{p}}=M_{\text {Earth }}$ and radius $R_{\mathrm{p}}=R_{\text {Earth }}$ but without an intrinsic magnetic field since it would result in strong magnetic fields that the simulation model can not handle (see section 2.2).

The simulation box has dimensions $10 R_{\mathrm{p}} \times 16 R_{\mathrm{p}} \times 20 R_{\mathrm{p}}$ and is divided into $73 \times$ $117 \times 147$ approximately cube-shaped cells, each cell with a width of $0.14 R_{\mathrm{p}}$. The planet is located $0.5 R_{\mathrm{p}}$ downstream of the center. The reason for the irregular geometry is to minimize the size of the simulation box while at same time including the interesting regions and extending the box in directions where the boundaries otherwise cause artefacts.

The time step size is

$$
\Delta t=0.04\left(\omega_{\mathrm{g}, \mathrm{H}^{+}}\right)^{-1}
$$

where $\omega_{\mathrm{g}, \mathrm{H}^{+}}$is the typical gyration frequency for hydrogen ions, i.e. the background stellar wind value. The simulations have run for a time equivalent to an undisturbed stellar wind

\footnotetext{
${ }^{2}$ Primary transit is when a planet passes in front of its host star and blocks out some of the starlight as seen from the Earth. See section 1.2
} 


\begin{tabular}{lcc}
\multicolumn{1}{c}{ Parameter } & & Value \\
\hline \hline Velocity & $v_{\mathrm{sw}, 0}$ & $300 \mathrm{~km} \mathrm{~s}^{-1}$ \\
Number density & $n_{\mathrm{sw}, 0}$ & $1050 \mathrm{~cm}^{-3}$ \\
IMF/magnetic field & $B_{\mathrm{sw}, 0}$ & $6 \mathrm{nT}$ \\
Velocity-IMF angle & $\alpha_{\mathrm{sw}, 0}$ & $60^{\circ}$ \\
Ion temperature & $T_{\mathrm{sw}, \mathrm{i}, 0}$ & $500000 \mathrm{~K}$ \\
Electron temperature & $T_{\mathrm{sw}, \mathrm{e}, 0}$ & $250000 \mathrm{~K}$ \\
\hline Alfvénic Mach number & $M_{\mathrm{A}}$ & 74 \\
Magnetosonic Mach number & $M_{\mathrm{ms}}$ & 2.7 \\
\hline \hline
\end{tabular}

Table 3.1: Stellar wind parameters used in the expanding atmospheres simulation runs.

passing through the simulation box more than seven times at which point they have all reached a quasistationary state.

\subsubsection{Stellar wind parameters}

We choose to work with a stellar wind similar to that from the Sun at a distance of $0.1 \mathrm{AU}$ and thus calculate the corresponding stellar wind parameters using the methods already described in section 2.4. The actual stellar wind at 0.1 AU for a Sun-like star can in principle be both subsonic or supersonic depending on the exact parameters (Preusse et al. 2005, 2007). However, working in a weak magnetic limit clearly puts us in the supersonic regime.

We use a higher stellar wind-IMF angle of $\alpha_{\mathrm{sw}, 0}=60^{\circ}$ than would be expected in an orbit at $r=0.1$ AU to ensure that we stay in the more familiar quasiperpendicular interaction regime where we can concentrate on the consequences of the expanding atmosphere and ionosphere instead of unintentionally triggering phenomena uniquely associated with a quasiparallel case ${ }^{3}$ (see section 5.1).

All stellar wind parameters are summarized in Table 3.1 .

\subsubsection{Ionospheric parameters}

The plasma hybrid simulation model implements an ionosphere as a volume in the simulation box where ionospheric plasma is continuously injected according to some chosen production profile and with some chosen temperature. The imagined neutral atmosphere which generates the ionosphere is not part of the hybrid model and is not simulated. In the special case of an expanding atmosphere we must also recognize that since the underlying neutral atmosphere is expanding radially outwards, ionospheric plasma will have to be injected into the simulation box with an initial radial bulk velocity.

\footnotetext{
${ }^{3}$ In principle of course, we could also attribute this to the natural diversity of host stars and planet systems instead of insisting on imitating the Sun's solar wind. Although we do not explore it in this work, exoplanets in elliptical close-in orbits would experience varying stellar wind-IMF angles over the course of their orbits which could also lead to greater stellar wind-IMF angles. Indeed, the known population of exoplanets, does display many exoplanets with eccentricities $e$ greater than we are used to in the Solar System, up to $e \sim 0.5$ for $r \sim 0.1 \mathrm{AU}$ (see e.g. http://exoplanet.eu).
} 
In the ideal case we would have an atmospheric model from which we would calculate the ion production rate profile, initial radial velocity profile and temperature based on photoionization, precipitation of energetic particles etc. applied to the known underlying neutral atmosphere. With the help of these profiles we could then inject ionospheric plasma into our simulation box. Unfortunately, modeling a hydrodynamically expanding atmosphere is a very nontrivial task. This is due to several unknowns and a number of very special physical effects, e.g. uncertain stellar conditions and atmospheric composition, a very intense dayside (i.e. asymmetric) photoionization and heating, uncertain atmospheric chemistry which complicates the calculation of the heat balance which in turn influences the expansion, atmospheres (exobases) which reach up to the very small Roche lobe and the difficulty of modeling the expansion behavior of an almost, but not quite, collisionless upper atmosphere (see e.g. Yelle 2004, Lecavelier des Etangs et al. 2004, Tian et al. 2005, Seager et al. 2005).

For these reasons we instead choose to postulate the existence of a spherically symmetric plasma producing region surrounding the planet where we inject hydrogen plasma with a postulated initial radial velocity $u$. The trivial way to do this would be with a thin shell coinciding with the simulated planet surface. This however would not mirror the fact that hydrodynamically expanding atmospheres, as opposed to "ordinary" atmospheres, extend to altitudes of the order of $R_{\mathrm{p}}$ and thus have very high-up ionospheres.

The observations of close-in extrasolar giant planet HD 209458 b seem to imply that hydrogen leaves the planet at $\sim 100 \mathrm{~km} \mathrm{~s}^{-1}$ in the starward direction (Vidal-Madjar et al. $2003)$ although it has later been disputed that it is due to hydrodynamic expansion (Holmström et al. 2008). Efforts to model hydrodynamic expansion, both for HD $209458 \mathrm{~b}$ and other cases, have only reproduced expansion velocities of $\sim 1-10 \mathrm{~km} \mathrm{~s}^{-1}$ at altitudes of $r \sim 2 R_{\mathrm{p}}$ (Yelle 2004, Tian et al. 2005). Since we are interested in the more extreme cases, we choose to work with expansion velocities up to the higher range of velocities, i.e. $100 \mathrm{~km} \mathrm{~s}^{-1}$.

Instead of just using one simulation run, we choose to use a series of three simulation runs which we can then compare. All three simulation runs are identical except for the expansion velocity: 1.) stationary atmosphere with an initial radial ionospheric plasma velocity $u=0 \mathrm{~km} \mathrm{~s}^{-1}$ which we use both as a reference case and to describe a very slowly expanding atmosphere, 2.) expanding atmosphere with $u \approx 50 \mathrm{~km} \mathrm{~s}^{-1}$ and 3.) high-speed atmosphere with $u \approx 100 \mathrm{~km} \mathrm{~s}^{-1}$. Note that we here speak of imagined neutral atmospheres which are not modeled in the simulations but are still reflected through the initial radial bulk velocity of the injected ionospheric plasma.

The initial ionospheric plasma velocity we use is

$$
u(r)=\frac{c_{1}}{r^{2}} \exp \left(-\frac{c_{2}}{r}\right)
$$

where $u(r)$ is the initial radial bulk velocity of ionospheric plasma being injected inside the simulation box at a distance $r$ from the center of the planet. $c_{1}$ is a constant with different values for the different simulation runs: $c_{1}=0$ for the stationary atmosphere run, $c_{1}=3.75 \cdot 10^{20} \mathrm{~m}^{3} \mathrm{~s}^{-1}$ for the expanding atmosphere run and $c_{1}=7.5 \cdot 10^{20} \mathrm{~m}^{3} \mathrm{~s}^{-1}$ for high-speed atmosphere run. $c_{2}=4.8 \cdot 10^{7} \mathrm{~m}$ is another postulated constant with the same value for all three simulation runs. 
The ionospheric production rate $Q(r)$ is given by

$$
Q(r)=c_{3} \exp \left[-\left(\frac{r-r_{Q, \max }}{\Delta r_{Q}}\right)^{2}\right] \exp \left[\frac{c_{2}}{r}\right]
$$

where $c_{3}=7.6 \cdot 10^{6} \mathrm{~m}^{-3} \mathrm{~s}^{-1}, r_{Q, \max }=2 R_{\mathrm{p}}$ and $\Delta r_{Q}=0.3 R_{\mathrm{p}}$ are all constants. This profile is set to zero outside the range $R_{\mathrm{p}}<r<3 R_{\mathrm{p}}$. The resulting integrated total production rate is $2.46 \cdot 10^{30} \mathrm{~s}^{-1}$.

The above given profiles produce an ion production peak at $r \approx r_{Q, \max }$ and initial radial ion bulk velocities of $u=0 \mathrm{~km} \mathrm{~s}^{-1}, u \approx 50 \mathrm{~km} \mathrm{~s}^{-1}$ and $u \approx 100 \mathrm{~km} \mathrm{~s}^{-1}$ at $r=r_{Q \text {, max }}$ as advertised and as can be seen in Fig. 3.1. Although somewhat arbitrary, the described profiles intentionally have the properties of both describing a high and "thick" ionosphere and of generating plasma at different velocities at different altitudes, all features that could be expected from the ionosphere of an expanded atmosphere although we have not tried quantify them here.

One could argue that in principle, atmospheres with so different outflow velocities as in our three simulation runs must be in very different physical states and therefore not have the same ionospheric profiles. To take such differences into account however would imply modeling the hydrodynamically expanding atmosphere which we want to avoid. We instead choose to study the consequences of changing only one variable in the description of the ionosphere, namely the initial ionospheric bulk velocity, implicitly described by the constant $c_{1}$ in Eq. 3.2.

Finally, thermospheric temperatures of giant close-in exoplanets such as HD $209458 \mathrm{~b}$ are expected to have temperatures of $T \sim 10^{4} \mathrm{~K}$ (Yelle 2004, Seager et al. 2005). Although we are not modeling a giant planet here we use this as a motivation for giving our ionospheric ions an initial temperature of $T_{\mathrm{i}}=10000 \mathrm{~K}$. We set the initial electron temperature to $T_{\mathrm{e}} \sim 2 T_{\mathrm{i}}$. The initial electron temperature can however not be set to a single definitive value since it effectively correlates with density due to the description of electrons as an adiabatic fluid (see Eq. 2.3) and since ionospheric plasma is simultaneously injected into regions with different densities.

Given the stellar wind in the preceding section, the characteristic gyration radius of picked up ionospheric hydrogen ions now becomes

$$
r_{\mathrm{g}, \mathrm{H}^{+}}=\frac{m_{\mathrm{p}} v_{\mathrm{sw}, 0}}{e B_{\mathrm{sw}, 0}} \approx 0.082 R_{\mathrm{p}}=0.60 \text { cells } .
$$

Gyrational effects can therefore not be resolved except possibly in regions with very weak magnetic field.

\subsection{Results}

The simulation results for the three simulation runs are illustrated in Figs. 3.2, 3.3, 3.4, 3.5 and 3.6 . Fig. 3.2 shows three-dimensional overviews of the ionospheric density for each simulation run. Figs. 3.3 and 3.4 depict the magnetic field, the plasma densities and the ionospheric plasma velocity plotted on equatorial and polar plane cross sections respectively and with one column of smaller figures for every simulation run. Fig. 3.5 shows a plot of the magnetic field $y$ component and both plasma densities and velocities 


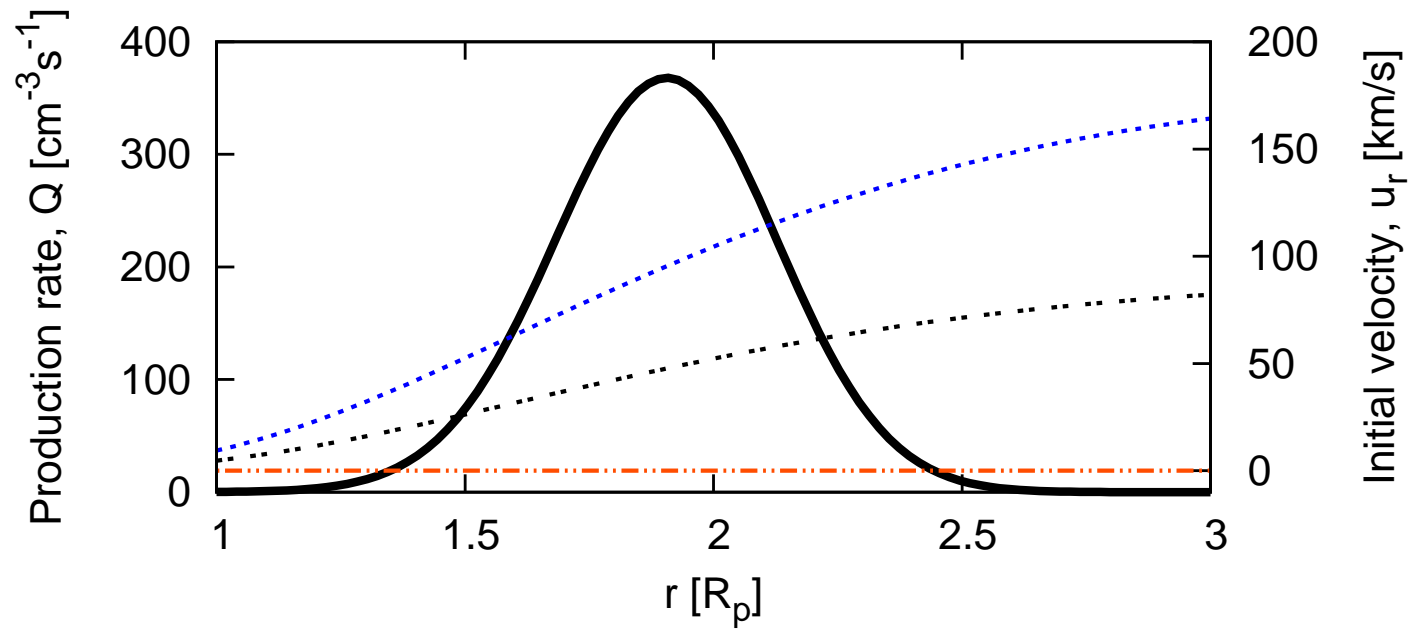

Figure 3.1: Ionospheric production rate profile used in all simulation runs and three initial radial velocity profiles, one for each of the three simulation runs. Black thick solid line: production rate of ionospheric ions. Red dash-dot-dotted line: initial velocity profile for the stationary atmosphere simulation run. Black double-dotted line: initial velocity profile for the expanding atmosphere simulation run. Blue dotted line: initial velocity profile for the high speed atmosphere simulation run.

for the entire $x$ axis. Fig. 3.6 similarly shows a plot of the pressure components for the dayside $x$ axis.

We start with the overall picture in Figs. 3.3 and 3.4 and recognize in all three runs almost all of the generic features we expect for a supersonic stellar wind interaction with the ionosphere of a planet without an intrinsic magnetic field. Since no information (perturbations) can travel upstream of a supersonic plasma flow, the stellar wind interaction begins suddenly when the stellar wind passes through the bow shock, the thin parabolic surface upstream of the planet where the stellar wind plasma is very suddenly slowed down and heated by the excess kinetic energy: the plasma is shocked. This is very visible as a jump in the stellar wind density and magnetic field, Figs. $3.3 \mathrm{a}-\mathrm{f}$ and $3.4 \mathrm{a}-\mathrm{f}$. Immediately downstream of the bow shock lies the magnetosheath, the region where the stellar wind is diverted around the ionosphere and the planet. This is very visible due to its overall high stellar wind density. The boundary between the stellar wind plasma in the magnetosheath and the ionospheric plasma in turn forms another fairly well-defined surface called the ion composition boundary (ICB) in Figs. 3.3 d-i and 3.4d-i (see e.g. Boesswetter et al. 2004, Simon et al. 2007a). This is not to be confused with another closely related term, the ionopause where the ionospheric pressure is equal to the incident stellar wind pressure (Kivelson and Russell 1995). The bulk of the ionospheric plasma is generated behind the ICB and is subsequently either forced downstream or toward the planet and never mixes with the stellar wind to any greater extent ${ }^{4}$.

\footnotetext{
${ }^{4}$ It must be understood that physically, the plasma above the planetary surface coexists with the very thin upper layers of the neutral atmosphere which, when collisions are negligible, does not interact with the stellar wind since it is not charged. This neutral atmosphere can very well extend above the ICB or ionopause and lead to the generation of ionospheric plasma there.
} 
Furthermore, as magnetic field lines are convected with the stellar wind through the bow shock the magnetic field is enhanced in parallel with the increased density since the magnetic field is frozen-in. As the stellar wind is afterwards diverted around the sides of the planet the convected magnetic field lines come to naturally drape around the obstacle as seen in the equatorial plane in Figs. $3.3 \mathrm{a}$-c. This leads to the downstream formation of two so called lobes, defined by the opposite directions of the magnetic field and separated by a current sheet, named so since the reversal of the magnetic field within a small region implies a current due to Ampère's law (Eq. 2.5). However, as a first look at the actual magnetic field in Figs. 3.3 a-c reveals, this current sheet is in our case really only a sheet in the stationary atmosphere run. It can be worth mentioning that the tail region with the weak magnetic field in Fig. $3.4 \mathrm{a}$ is the very same current sheet but with the cross section cut parallel to the sheet instead of through it.

The very existence of an ionospheric plasma surrounding the planet is important in the above picture since the ionospheric conductivity, and implicitly the induced magnetic field, prevents the magnetic field convected with the stellar wind from diffusing into the obstacle. It can thus divert the stellar wind without absorbing it. It is therefore the ionosphere which is the obstacle to the stellar wind and what the stellar wind primarily reacts to, not the planet itself.

It can also be noted that the convected IMF is what breaks the symmetry of the interaction and what creates the difference between the equatorial and polar planes. The stellar wind interaction would ideally have been cylindrically symmetric had it not been for the orientation of the incoming stellar wind magnetic field. Therefore, all deviations from a mirror symmetry in all the cross sections should in principle be due to the existence of a stellar wind magnetic field component perpendicular to the undisturbed stellar wind velocity.

Starting by looking at the bow shock in all three runs, we can first note in Fig. 3.5 that the jump in stellar wind density and magnetic field follow each other very nicely as predicted by the frozen-in condition. Similarly, the relative importance of the various stellar wind pressure components in Fig. 3.6 abruptly change at the bow shock.

Looking at the same variables in the polar cross sections, Figs. $3.4 \mathrm{a}-\mathrm{f}$, the same seems to hold true for the entire bow shock. For the equatorial plane, Figs. $3.3 \mathrm{a}-\mathrm{f}$, the jump in magnetic field disappears on the flanks while the stellar wind density jump remains. On at least the negative $y$ flank this can be explained by the geometry, since the shock here approaches a parallel shock (see section 5.1), implying that the magnetic field can not be enhanced by the bow shock since the enhancement really only applies to the component perpendicular to the shock. This effect does not exist on the positive $y$ flank but it does at least explain the asymmetry in that plane.

Since the simulation box is smaller in the $y$ direction than in the $z$ direction and the magnetic field vector is set to be equal to the undisturbed stellar wind value on all the simulation box boundaries except the downstream boundary one could be led to believe that this would somehow cause trouble for the magnetic field in the equatorial plane, Fig. 3.3 a-c. One could imagine that the forced, weaker field on the box boundary would somehow force the enhanced magnetic field to be reduced when approaching the edges. Testing with larger boxes have however shown that this is unlikely to cause any greater effect, especially on the enhancement of the magnetic field behind the actual bow shock.

As mentioned earlier, all three simulation runs are identical with the exception of the 

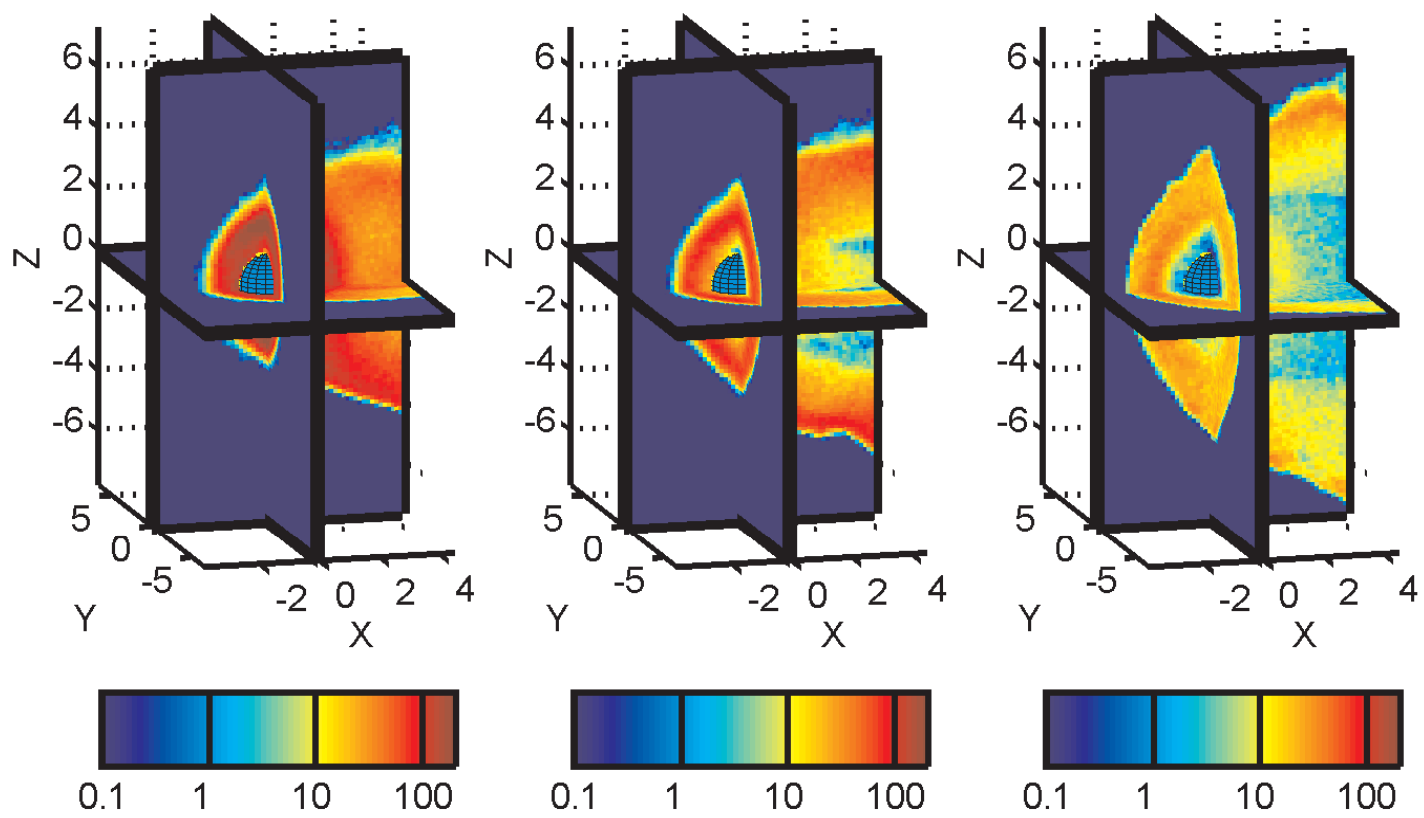

Figure 3.2: Three-dimensional overview of simulations results for the ionospheric density in the stationary atmosphere simulation run (left), expanding atmosphere simulation (middle) and high speed atmosphere simulation run (right). The density is normalized to the background stellar wind density. Parts of the simulation box with zero ionospheric density have been removed for better overview.

initial radial velocity given to each ionospheric particle upon insertion in ionosphere. The most obvious consequence of this, as we can see when comparing the different runs in Figs. $3.3 \mathrm{~d}-\mathrm{i}$ and $3.4 \mathrm{~d}-\mathrm{i}$, is that dynamic pressure is added to the ionosphere, thus forcing the stellar wind and the ICB farther upstream and farther out on the flanks, effectively making the ionosphere a larger obstacle to the stellar wind. This in turn increases the size and thickness of the entire magnetosheath. The displacement of the ICB is also visible in Fig. 3.5, where the ICB is visible approximately as the point where the density of the two plasmas is the same. The ICB is displaced $\sim 0.5 R_{\mathrm{p}}$ upstream and the bow shock an entire $1.5 R_{\mathrm{p}}$ upstream, signifying the growth of the magnetosheath and in turn a sign of the increasing size of the effective obstacle.

Fig. 3.6 of course presents a similar picture but in terms of pressure components: the ionopause and bow shock are pushed upstream with increasing initial ionospheric radial velocity. Here we see however how the sum of the various pressure components, including dynamic pressure, is remarkably constant on the entire dayside $x$ axis in all three simulation runs and over both the bow shock and the ionopause. A closer look at Fig. 3.6 however shows that dynamic pressure is in reality a very small part of the total pressure as opposed to what one could naively expect. Instead, it is the ionospheric thermal ion pressure that dominates the ionosphere, also for the expanding atmosphere run and the high-speed atmosphere run. Also Fig. 3.5 verifies this: the actual ionospheric bulk velocity is in fact nowhere near the initial velocities of $u \approx 50 \mathrm{~km} \mathrm{~s}^{-1}=\frac{1}{6} v_{\mathrm{sw}, 0}$ for the expanding atmosphere run or $u \approx 100 \mathrm{~km} \mathrm{~s}^{-1}=\frac{1}{3} v_{\mathrm{sw}, 0}$ for the high-speed atmosphere 


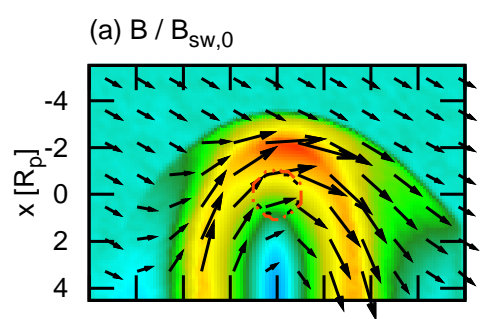

(d) $n_{\mathrm{sw}} / \mathrm{n}_{\mathrm{sw}, 0}$

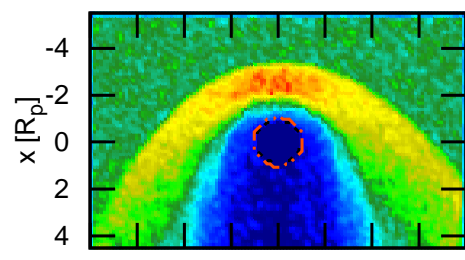

(g) $n_{i} / n_{s w, 0}$

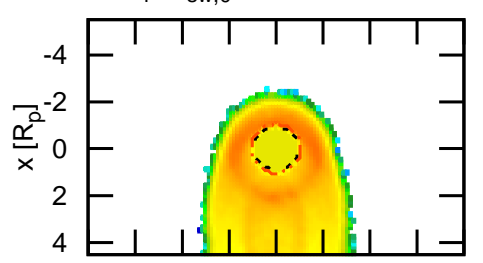

(j) $u_{i} / u_{s w, 0}$

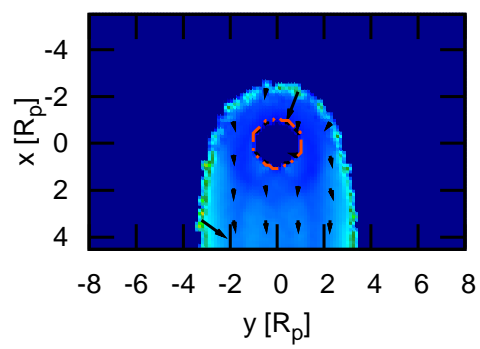

(b) B / $\mathrm{B}_{\mathrm{sw}, 0}$

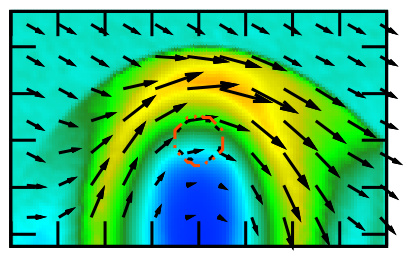

(e) $n_{s w} / n_{s w, 0}$

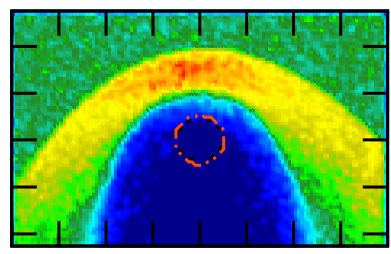

(h) $\mathrm{n}_{\mathrm{i}} / \mathrm{n}_{\mathrm{sw}, 0}$

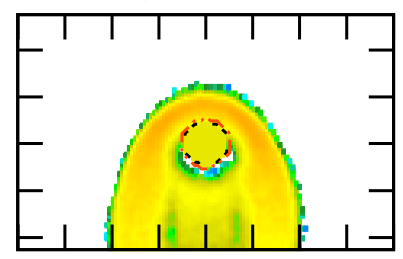

(k) $u_{i} / u_{s w, 0}$

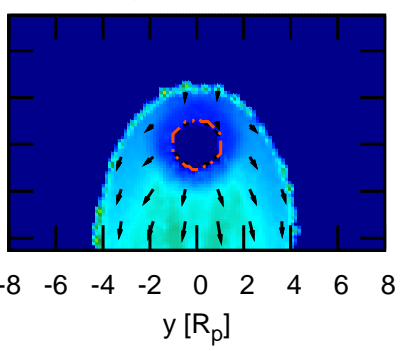

(c) B / $\mathrm{B}_{\mathrm{sw}, 0}$

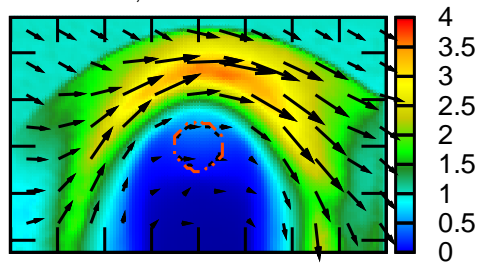

(f) $n_{s w} / n_{s w, 0}$

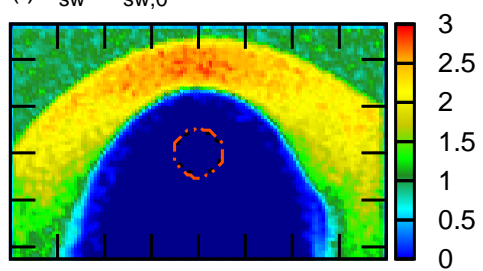

(i) $\mathrm{n}_{\mathrm{i}} / \mathrm{n}_{\mathrm{sw}, 0}$

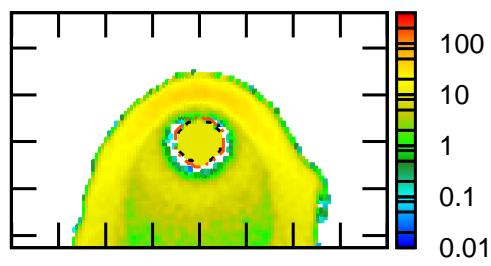

(I) $u_{i} / u_{s w, 0}$

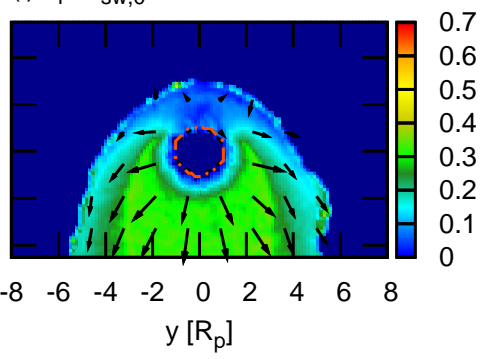

Figure 3.3: Simulation results, depicted as equatorial cross sections through the simulation box. The left column figures refer to the stationary atmosphere run, the middle column to the expanding atmosphere run and the right column to the high-speed run. The first row (Figs. a-c) depicts the magnetic field, the second row (Figs. d-f) the stellar wind number density, the third row (Figs. g-i) the ionospheric plasma number density and the fourth row (Figs. $j-1$ ) the ionospheric velocity. All quantities have been normalized using the corresponding undisturbed stellar wind values.

run that one would naively expect. The typical distance a hydrogen ion can travel before being diverted by the magnetic field is the gyration radius

$$
r_{\mathrm{g}}=\frac{m_{\mathrm{p}} v}{e B}
$$

where $v$ is the speed of an individual ion relative to the bulk flow of the ambient plasma. When ions are injected into the ambient dayside ionosphere in the high-speed atmosphere run, we have $v \approx 100 \mathrm{~km} \mathrm{~s}^{-1}$ and $B \sim 3 B_{\mathrm{sw}, 0}$ implying a gyration radius of $r_{\mathrm{g}} \sim 10^{-2} R_{\mathrm{p}}$. 
(a) B / $\mathrm{B}_{\mathrm{sw}, 0}$

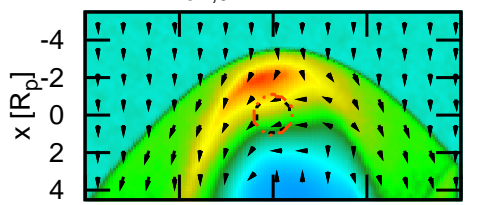

(d) $n_{s w} / n_{s w, 0}$
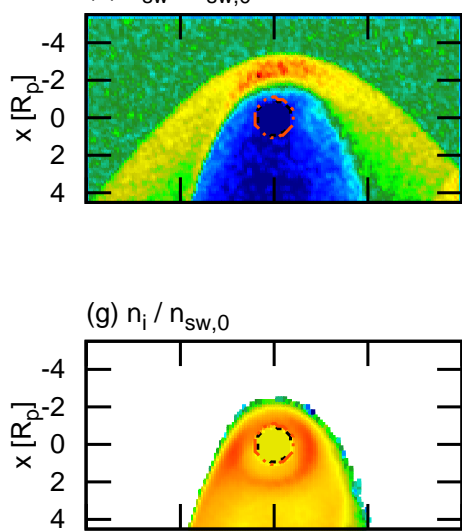

(j) $u_{i} / u_{s w, 0}$

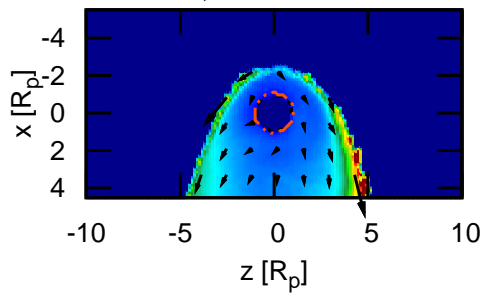

(b) B / $\mathrm{B}_{\text {sw, } 0}$

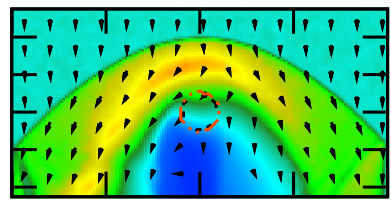

(e) $n_{s w} / n_{s w, 0}$
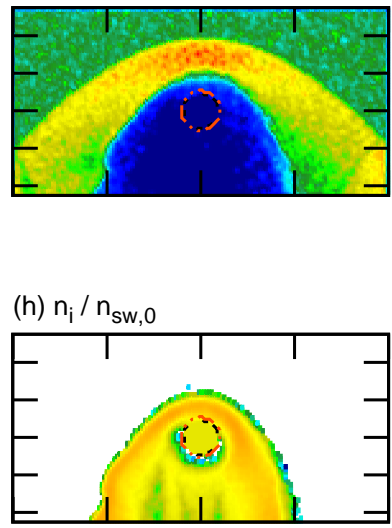

(k) $u_{i} / u_{s w, 0}$

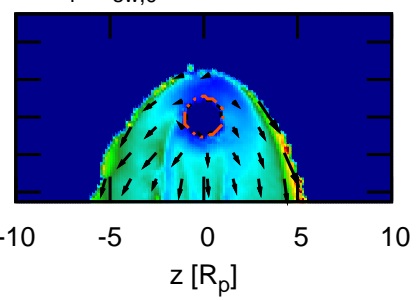

(c) B / $\mathrm{B}_{\mathrm{sw}, 0}$
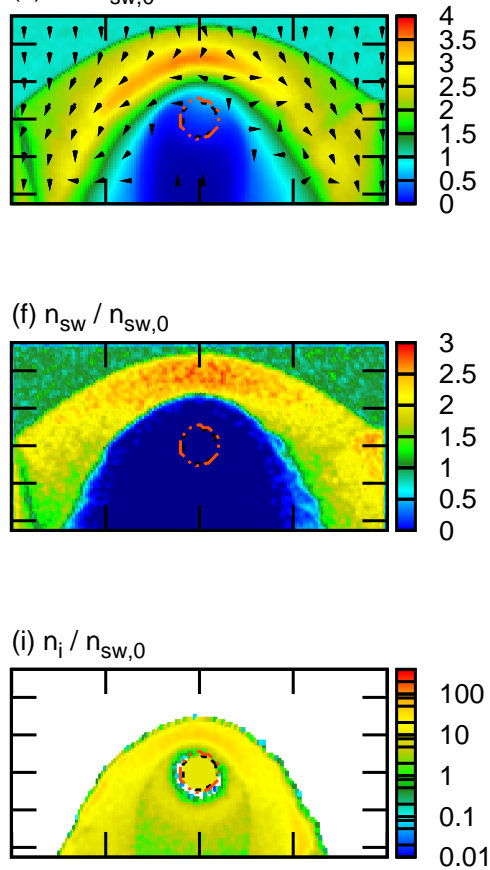

(I) $u_{i} / u_{s w, 0}$

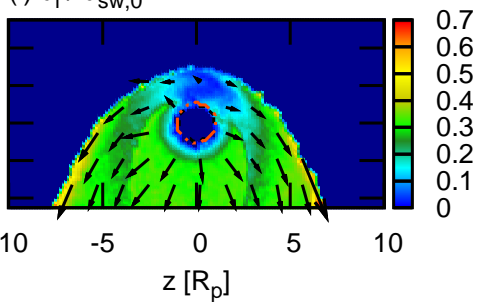

Figure 3.4: Simulation results, depicted as polar cross sections through the simulation box. The left column figures refer to the stationary atmosphere run, the middle column to the expanding atmosphere run and the right column to the high-speed run. The first row (Figs. a-c) depicts the magnetic field, the second row (Figs. d-f) the stellar wind number density, the third row (Figs. g-i) the ionospheric plasma number density and the fourth row (Figs. j-1) the ionospheric velocity. All quantities have been normalized using the corresponding undisturbed stellar wind values.

This means that the bulk motion is immediately thermalized after injection and that the expected dynamic pressure is transformed into thermal ion pressure while the bulk momentum from the injected ions is transferred to the surrounding plasma.

Looking at the ionosphere itself in Figs. $3.3 \mathrm{~g}-\mathrm{i}$ and $3.4 \mathrm{~g}-\mathrm{i}$ we can first note how the overall ionospheric density grows thinner with increasing expansion velocity as plasma is more swiftly transported away from the planet and out of the simulation box. We can also see the ionosphere-dominated tail region widening and changing from being more or less homogeneous to being composed of a thick layer in contact with the stellar wind and an 

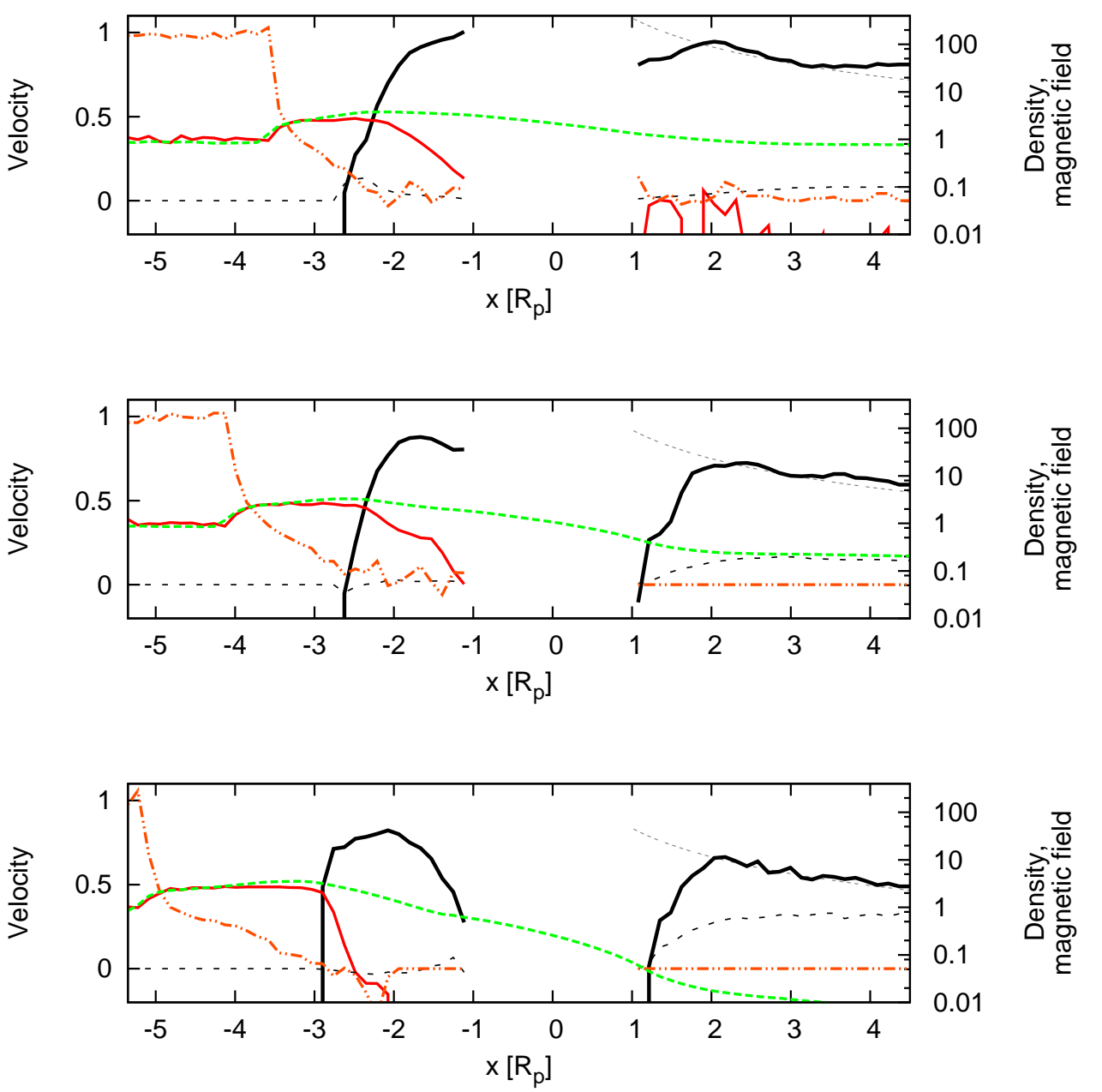

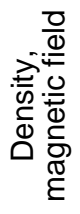

Figure 3.5: Comparison of simulation results for the stationary (top), expanding (middle) and high-speed (bottom) atmosphere runs in the form of quantities plotted as curves on the $x$ axis, parallel to the undisturbed stellar wind which travels in the $x+$ direction. Red solid line: stellar wind density. Red dash-dot-dotted line: stellar wind velocity in the $x$ direction. Black solid line: ionospheric density. Black dashed line: ionospheric velocity in the $x$ direction. Black thin dashed line (only nightside): "ideal ionospheric expansion density" i.e. the density assuming an ideal and spherically symmetric ionosphere with constant radial outflow velocity of $u=v_{\text {th,i }} \approx 13 \mathrm{~km} \mathrm{~s}^{-1}, u=50 \mathrm{~km} \mathrm{~s}^{-1}$ and $u=100 \mathrm{~km} \mathrm{~s}^{-1}$ respectively. Green dashed line: magnetic field in the $y$ direction. All values are normalized to the background stellar wind values. The very uneven stellar wind density on the nightside in the stationary atmosphere run (top) is due to the fact that few superparticles can not describe a thin smooth plasma density.

inside region which in the case of the high-speed atmosphere run seems to be undisturbed enough to allow the nightside ionosphere to locally expand while maintaining the initial spherical symmetry as can be seen in both the density and velocity plots in Figs. 3.31 and 3.4il. This is confirmed again in Fig. 3.5 where the magnitude of both ionospheric 

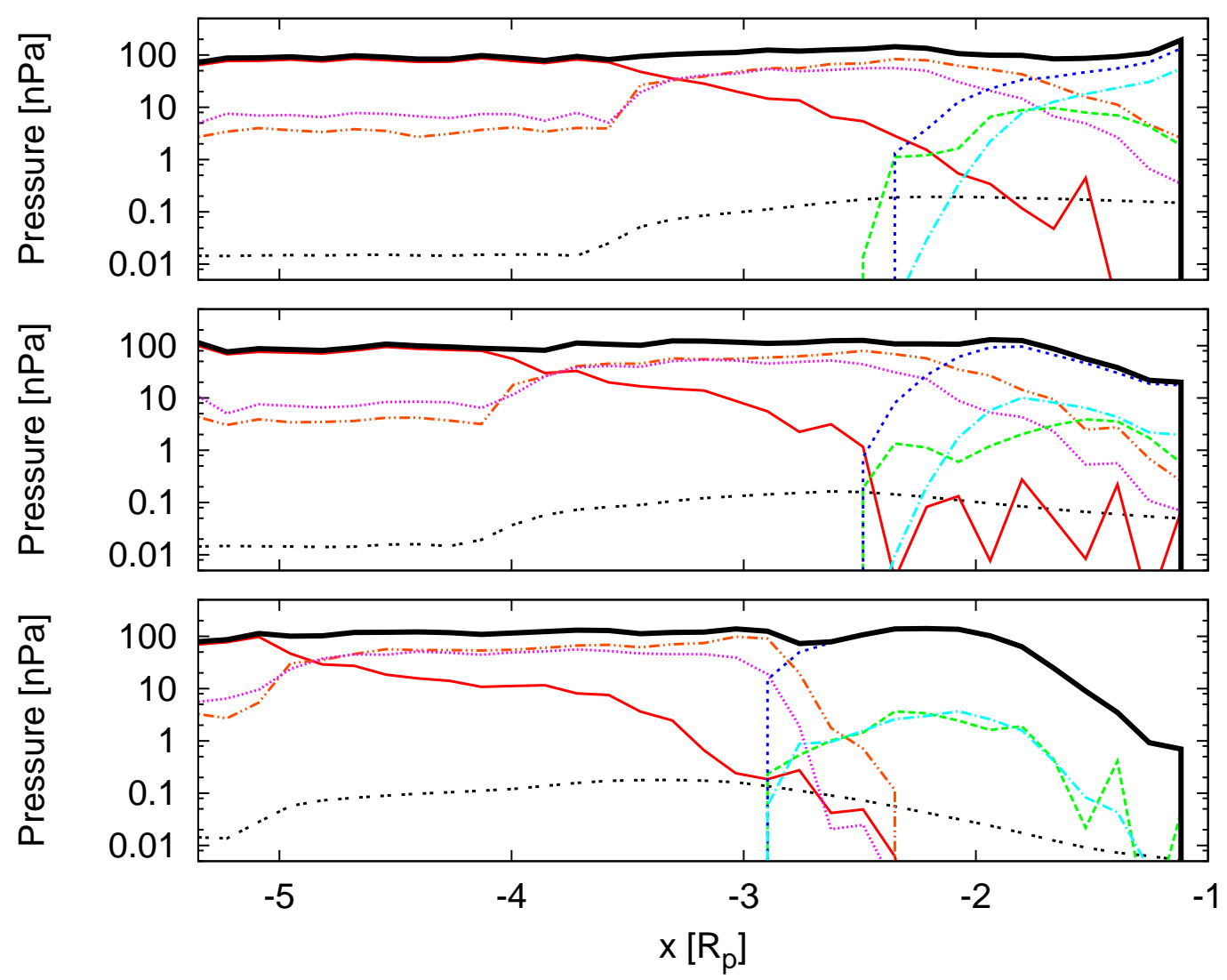

Figure 3.6: The pressure components on the dayside $x$ axis for the stationary (top), expanding (middle) and high-speed (bottom) run. Black solid line: total pressure (sum of all other given components). Black dash-dashed line: magnetic pressure. Red solid line: stellar wind dynamic pressure. Red dash-dot-dotted line: stellar wind thermal ion pressure. Violet dotted line: stellar wind thermal electron pressure. Green dashed line: ionospheric dynamic pressure. Dark blue dash-dotted line: ionospheric thermal ion pressure. Cyan dash-dotted line: ionospheric thermal electron pressure.

density and velocity on the nightside are what can be expected from such an "ideal" spherically symmetric expansion, i.e. $n_{\text {is }} \propto 1 /\left(r^{2} v_{\text {is }}\right)$ and $v_{\text {is }} \approx u \approx 100 \mathrm{~km} \mathrm{~s}^{-1}=\frac{1}{3} v_{\text {sw }}$. In fact, also the expanding atmosphere run $\left(u \approx 50 \mathrm{~km} \mathrm{~s}^{-1}\right)$ and the stationary run (using $u=v_{\mathrm{th}, \mathrm{i}} \approx 13 \mathrm{~km} \mathrm{~s}^{-1}$ instead of $u=0 \mathrm{~km} \mathrm{~s}^{-1}$ ) satisfy this condition fairly well confirming that the tail is very undisturbed by the stellar wind.

The expansion also transforms the thin current sheet, separating the two rear lobes of magnetic field in opposite directions in Fig. $3.3 \mathrm{a}$, into the wide tail region with a much lower magnetic field strength than the background stellar wind in Fig. 3.3k.

Figs. 3.3 d-f and 3.4d-f reveal that the ICB is changed not only in its form by the expanding ionosphere but also in how much trace amount of stellar wind that manages to penetrate through it and into the $\sim 0.6 R_{\mathrm{p}}$ thick ionosphere, including the tail region. One can there see that higher expansion speed leads to less mixing of ionosphere and stellar wind, something which can be understood by comparing the location of the substellar ICB in Fig. 3.5 with where ionospheric plasma is injected into the simulation in Fig. 3.1. If the 
ICB is inside this region then some ionospheric plasma will inevitably be injected above the ICB and directly into the stellar wind thus leading to more mixing of the plasmas than mere diffusion etc. would imply. In the expanding and high-speed atmosphere runs however, the ICB is pushed to higher altitudes reducing the effect of this mixing in the first place. This picture is confirmed when we look at the overlap of stellar wind and ionospheric plasma in Fig. 3.5 which decreases when the expansion velocity increases. Since the mixed or non-mixed plasma close to the ICB is also transported away, roughly parallel to the ionopause, the mixing should roughly remain the same following the ICB downstream. Figs. $3.3 \mathrm{~d}-\mathrm{f}$ and $3.4 \mathrm{~d}-\mathrm{f}$ confirm this although it is a little uncertain in the case of Figs. 3.4 fe since the different velocities on the different sides of the ICB here seem to lead to the onset of a Kelvin-Helmholtz instability.

Scrutinizing the figures a little bit more reveals that there are more signs of KelvinHelmholtz instabilities in the form of small deformations on the downstream parts of the ICB, visible in Figs. $3.31 \mathrm{l}$ and $3.4 \mathrm{hk}$. Not all of these are obvious instabilities if one only studies the one single time step depicted in the figures in this work however.

We also do note the existence of a few artefacts in the form of reflected waves or hints thereof on the simulation box boundaries. This is particularly visible in the stellar wind density distribution in Fig. 3.4d-f. We judge that none of these have any significant influence on the results in this work.

We can also see that the convection of the magnetic field (the frozen-in approximation) is not perfect in our simulations since it does diffuse into the ionosphere, out of the stellar wind plasma as one can see when comparing Figs. $3.3 \mathrm{~d}-\mathrm{f}$ with $3.3 \mathrm{a}$-c. This happens despite us not having any explicit diffusivity but is instead likely due to the numerical smoothing which we are forced to apply to the electromagnetic fields in order to ensure numerical stability. This smoothing has an effect very similar to an artificial diffusivity. See section 2.2.

A last minor result is obtained by counting the number of ionospheric ions which are removed at the simulation box boundaries rather than at the surface of the planet. This number as a percentage of the total production rate gives a hint to how large a fraction of the ionosphere is lost to space instead of being "recombined" and returned to the lower atmosphere of the planet. We record that $90 \%$ of the ionospheric ions reach the simulation box boundaries for the stationary atmosphere run and virtually $100 \%$ for both the expanding atmosphere and high-speed atmosphere run. Not so surprisingly, expansion prevents ions from reaching the planetary surface again. 



\section{Standoff distance for expanding atmospheres}

\subsection{Introduction}

We have seen in chapter 3 that, not very surprisingly, an ionosphere where ions are produced with an additional radial velocity increases the size of the magnetosphere and increases the distance from the planet to the ionopause. This distance between planet and ionopause is very similar to the standoff distance $R_{\mathrm{s}}$ for planets with an intrinsic magnetic dipole moment. The standoff distance is then defined as the planetocentric distance to the substellar point on the magnetopause, the surface where magnetospheric pressure and stellar wind pressure balance each other.

Inspired by previously published analytical estimates of the standoff distance for planets with an intrinsic magnetic dipole moment in e.g. Griessmeier et al. (2007a), Kivelson and Russell (1995) we will here try to construct similar estimates applicable to planets with expanding ionospheres and compare the results with standoff distances derived from a set of simulations including the already presented simulations in chapter 3 . The results here are the preliminary results to be published in Griessmeier et al. (2010).

For simplicity, we will also use the term standoff distance to refer to the planetocentric distance to the substellar point on the ionopause for planets lacking an intrinsic dipole field.

\subsection{Analytical estimates of standoff distance}

Griessmeier et al. (2007a) estimates the standoff distance $R_{\mathrm{s}}$ for planets with a dipole magnetic field by using the above definition of magnetopause. By setting the dynamic pressure and thermal pressure of the incoming stellar wind (left-hand side) to be equal to the magnetic pressure caused by the planetary magnetic dipole (right-hand side) one obtains

$$
m_{\mathrm{p}} n_{\mathrm{sw}} v_{\mathrm{sw}}{ }^{2}+2 n_{\mathrm{sw}} k_{\mathrm{B}} T=\frac{\mu_{0} f_{0}^{2} \mathscr{M}^{2}}{8 \pi^{2} R_{\mathrm{s}}{ }^{6}}
$$

where $n_{\mathrm{sw}}$ and $v_{\mathrm{sw}}$ are the stellar wind density and velocity, $T$ is the temperature of the stellar wind, $f_{0}=1.16$ is a form factor for the magnetosphere (Voigt 1995) and $\mathscr{M}$ is the planetary magnetic dipole moment. The factor 2 for the stellar wind thermal pressure stems from treating ions and electrons as two separate particle species. The above relation implicitly assumes that the dipole moment is perpendicular to the stellar wind direction 
and that the contributions from IMF magnetic pressure and magnetospheric thermal pressure are negligible. Thanks to the distance dependence of the dipole field one can now trivially calculate the standoff distance from Eq. 4.1 assuming that all other quantities are known.

Since the simulations in this work deal with planets without an intrinsic magnetic dipole field, Eq. 4.1 is not directly applicable here. However, if the main ionospheric pressure is dynamic pressure, then this suggest an interesting modification of Eq. 4.1 for the case of expanding ionospheres and planets without an intrinsic magnetic field: replacing the magnetic pressure term with an ionospheric dynamic (and thermal) pressure term. We can do this by assuming that the ionospheric plasma is spherically symmetric and expands radially away from the planet with constant radial velocity $u$, i.e.

$$
n_{\mathrm{is}}=\frac{Q_{\mathrm{tot}}}{4 \pi r^{2} u}
$$

where $n_{\text {is }}$ is the ionospheric density, $Q_{\text {tot }}$ is the total ionospheric production rate and $r$ is the distance to the center of the planet. If we also modify Eq. 4.1 to distinguish between ion and electron pressures then we have an expression which we can use to estimate standoff distances and compare with our simulation results for expanding ionospheres from section 3.3 . The resulting relation for our own estimate of standoff distance, $R_{\mathrm{s}, 1}$, is then

$$
m_{\mathrm{p}} n_{\mathrm{sw}} v_{\mathrm{sw}}{ }^{2}+n_{\mathrm{sw}} k_{\mathrm{B}}\left(T_{\mathrm{sw}, \mathrm{i}}+T_{\mathrm{sw}, \mathrm{e}}\right)=\frac{Q_{\mathrm{tot}}}{4 \pi R_{\mathrm{s}, 1}{ }^{2} u}\left(m_{\mathrm{p}} u^{2}+k_{\mathrm{B}}\left(T_{\mathrm{is}, \mathrm{i}}+T_{\mathrm{is}, \mathrm{e}}\right)\right)
$$

where $T_{\mathrm{sw}, \mathrm{i}}, T_{\mathrm{sw}, \mathrm{e}}, T_{\mathrm{is}, \mathrm{i}}$ and $T_{\mathrm{is}, \mathrm{e}}$ are the initial stellar wind (sw) and ionospheric (is) temperatures for both ions (i) and electrons (e). We have also implicitly assumed that the ionosphere consists of hydrogen. Again, $R_{\mathrm{s}, 1}$, can easily be calculated from this expression assuming that all other quantities are known.

For reasons which will become clear in the discussion of results, we also define a standoff distance estimate

$$
R_{\mathrm{s}, 2}=\sqrt{C} R_{\mathrm{s}, 1}
$$

where $C=1.5$ is a constant. This can be interpreted as $R_{\mathrm{s}, 1}$ but calculated for a higher total ionospheric production rate $C Q_{\text {tot }}$.

\subsection{Standoff distances from simulations}

We have, in addition to the three simulation runs in chapter 3 , run three series of similar simulations to test the validity of the analytical estimates of standoff distance in the preceding section. Each series of simulations has been constructed by taking the expanding atmosphere run $\left(u \approx 50 \mathrm{~km} \mathrm{~s}^{-1}\right)$ from chapter 3 and rerunning the simulation for different values of one chosen input parameter. The parameters we have looked at are: initial ionospheric radial bulk velocity $u$, stellar wind velocity $v_{\mathrm{sw}}$ and stellar wind density $n_{\mathrm{sw}}$.

The standoff distance $R_{\mathrm{s}, \mathrm{sim}}$. in the simulation results is found by searching for the point on the dayside $x$ axis where

$$
n_{\mathrm{sw}} v_{\mathrm{sw}, \mathrm{x}}^{2}+n_{\mathrm{sw}} k_{\mathrm{B}}\left(T_{\mathrm{sw}, \mathrm{i}}+T_{\mathrm{sw}, \mathrm{e}}\right)=n_{\mathrm{is}} v_{\mathrm{is}, \mathrm{x}}^{2}+n_{\mathrm{is}} k_{\mathrm{B}}\left(T_{\mathrm{is}, \mathrm{i}}+T_{\mathrm{is}, \mathrm{e}}\right)
$$


is satisfied. Here $v_{\mathrm{sw}, \mathrm{x}}$ and $v_{\mathrm{is}, \mathrm{x}}$ are $x$ components of the stellar wind velocity and ionospheric velocity respectively. All quantities are local quantities as opposed to in the rest of this chapter. The magnetic pressure is too small to influence the result and it is also uncertain how one should include it since in our simulation runs the magnetic field diffuses over the ionopause anyway, see Figs. 3.5 and 3.6.

\subsection{Results}

The results are illustrated in Figs. 4.1 and $4.2 \mathrm{a}-\mathrm{b}$ in the form of standoff distance as a function of initial ionospheric radial velocity $u$, stellar wind density $n_{\text {sw }}$ and stellar wind velocity $v_{\mathrm{sw}}$ respectively. Fig. 3.6 from the results of the preceding chapter depicting the various pressure components on the dayside $x$ axis is also of some relevance. The exact parameter values and standoff distances are listed in Appendix A.

We begin by noting that all the standoff distances, resulting from both simulation results and analytical estimates, have the tendencies one can expect: standoff distance increases with initial radial ionospheric velocity in Fig. 4.1 and decreases with both increasing stellar wind density and stellar wind velocity in Figs. $4.2 \mathrm{a}-\mathrm{b}$. However, our original estimate $R_{\mathrm{s}, 1}$ consistently underestimates the value produced by simulations.

One important part of this dichotomy stems from that our expression for ionospheric pressure, i.e. the right-hand side of Eq. 4.3, can only be valid for altitudes above the layer where the ionospheric plasma is produced. Since the ionospheric production layer is centered around an altitude of $r \sim 1.9 R_{\mathrm{p}}$ and has a characteristic thickness of $0.6 R_{\mathrm{p}}$ (see Fig. 3.1) we should expect the best agreement for $R_{\mathrm{s}, \text { sim. }}>2.2 R_{\mathrm{p}}$ and progressively less agreement for lower altitudes. Interestingly enough though, when taking this into account and comparing with $R_{\mathrm{s}, \mathrm{sim}}$. for high values, $R_{\mathrm{s}, 1}$ does seem to be off by an approximately constant difference in both Fig. 4.1 and 4.2 b. The clear exception is Fig. 4.2 a, standoff distance as a function of stellar wind density.

This observation leads us to speculate that this difference is due to a higher effective ionospheric density as Fig. 3.5 indeed suggests if we compare the dayside ionospheric density with the plotted nightside "ideal ionospheric expansion density", equivalent to our calculated ionospheric density in Eq. 4.2. As mentioned before, we have also defined $R_{\mathrm{s}, 2}=\sqrt{C} R_{\mathrm{s}, 1}$ which can be interpreted as using a higher total ionospheric production rate. The exact value of $C=1.5$ used in Figs. 4.1 and $4.2 \mathrm{a}$-b has been chosen only to produce a fit between $R_{\mathrm{s}, 2}$ and $R_{\mathrm{s}, \mathrm{sim} \text {. }}$. We can now see that the fit for $R_{\mathrm{s}, 2}>2.2 R_{\mathrm{p}}$ is quite good in Figs. 4.1 and 4.2 b. The clear exception is again Fig. 4.2 a, standoff distance as a function of stellar wind density.

One hypothesis for why such a factor $C$ is necessary would be that the effective ionospheric plasma pressure is higher since the plasma also has to be transported away from the substellar region. The ionospheric dynamic pressure term in Eq. 4.3 basically assumes that ionospheric plasma travels towards the ionopause, stops and then suddenly disappears which is obviously not true.

Maybe even more important for this discussion is that as we concluded in section 3.3 the stellar wind at the ionopause is in practice not held back by dynamic pressure directly but by thermal pressure. The initially fast-moving ionospheric plasma is very quickly thermalized and the dynamic pressure is in practice replaced by thermal pressure as one 
can see in Fig. 3.6. This means that the characteristic bulk velocity of the dayside ionospheric plasma is lower, something which does however fit with the notion that dayside ionospheric plasma is transported away more slowly.

It thus remains to construct an accurate estimate of the standoff distance although there are hints that it should be possible. More simulations for standoff distances above $\sim 2.2 R_{\mathrm{p}}$ would of course be helpful in this cause.

The notion of a pressure balance for pressures on both sides of the ionopause is sound, but estimating those pressures is harder. The left-hand side of Eq. 4.3 is only the approximate stellar wind pressure at the substellar point on the ionopause since it does not take the divergence of the flow around the obstacle into account. Kivelson and Russell (1995) describes a method of relating the upstream solar wind dynamic pressure to the solar wind stagnation pressure on the magnetopause or ionopause using momentum conservation, Bernoulli's principle and the Rankine-Hugoniot relations. For the Earth this means that solar wind stagnation pressure on the magnetopause is about $11 \%$ smaller than in the upstream solar wind. It is interesting to note that a similar decrease in the left-hand side of Eq. 4.3 would imply a somewhat greater standoff distance. This might partly explain why $R_{\mathrm{s}, 1}$ consistently underestimates $R_{\mathrm{s}, \mathrm{sim}}$. Unfortunately, this method assumes that the upstream solar wind thermal pressure is a small fraction of the total pressure, about $1 \%$ at the Earth. This assumption is less well satisfied for us (about $7 \%$ of total pressure) and since the size of the correction for our parameters, $5-11 \%$, is of about the same magnitude as the error from neglecting the thermal pressure we can not use it. However, it is not inconceivable that this method can be generalized to include upstream thermal pressure and eventually be put to use here.

The pressure components in Fig. 3.6 are thus deceiving since we can not really expect the sum of the pressure components to be completely constant except for over the ionopause. This should be true for the ionospheric side too since the same argument behind the difference in pressure between stellar wind and ionopause applies there too. 


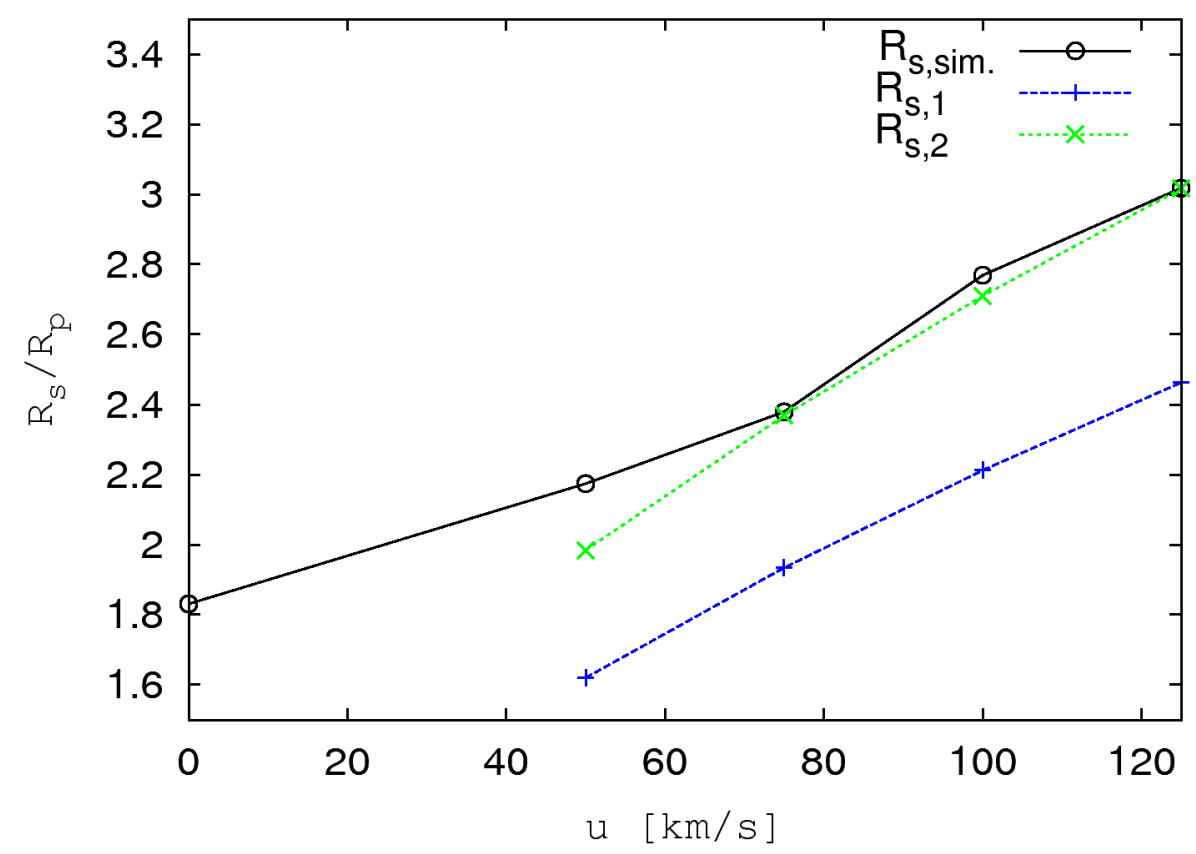

Figure 4.1: Standoff distance as a function of initial ionospheric radial velocity $u$, derived from simulations and analytical expressions. Black solid line, $R_{\mathrm{s}, \mathrm{sim}}$ : standoff distance calculated from simulation runs. Blue dashed line, $R_{\mathrm{s}, 1}$ : standoff distance calculated using Eq. 4.3. Green dashed line, $R_{\mathrm{s}, 2}=\sqrt{C} R_{\mathrm{s}, 1}$ for $C=1.5$. $R_{\mathrm{s}, 1}$ and $R_{\mathrm{s}, 2}$ are not defined for $u=0$. 
a)

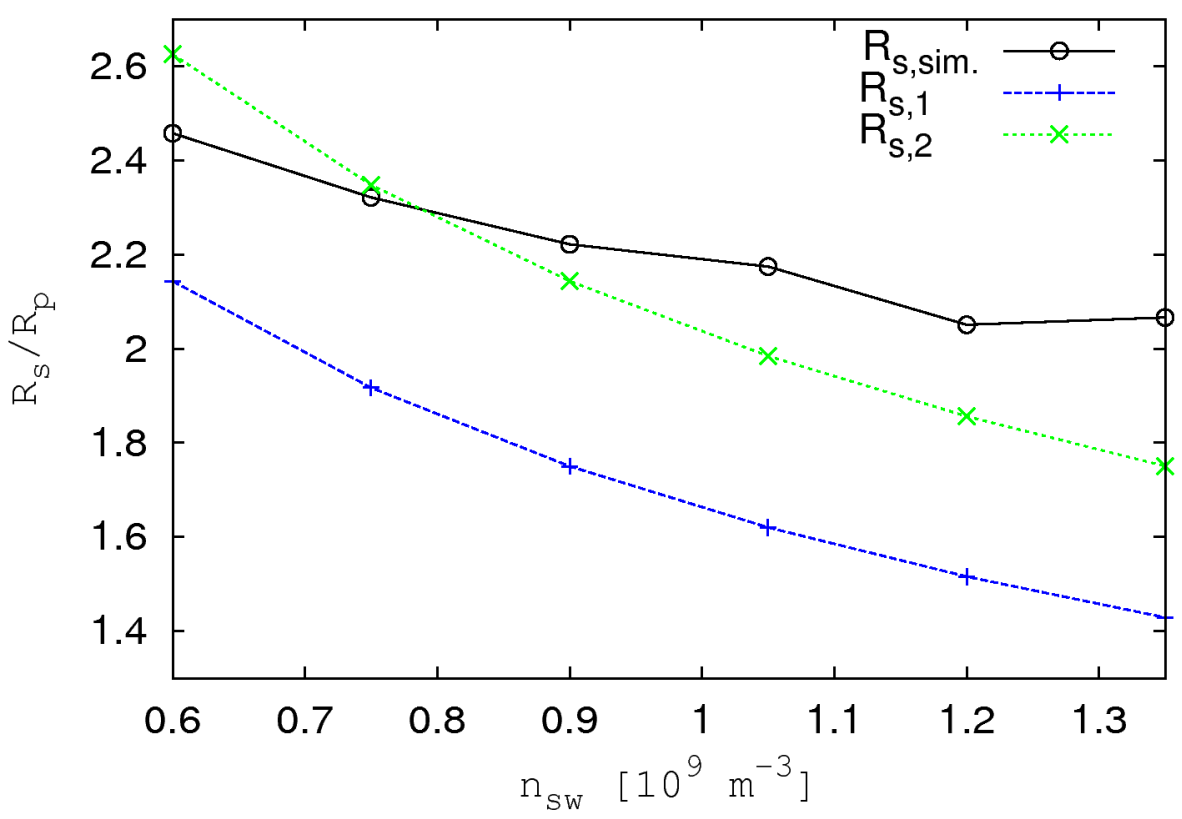

b)

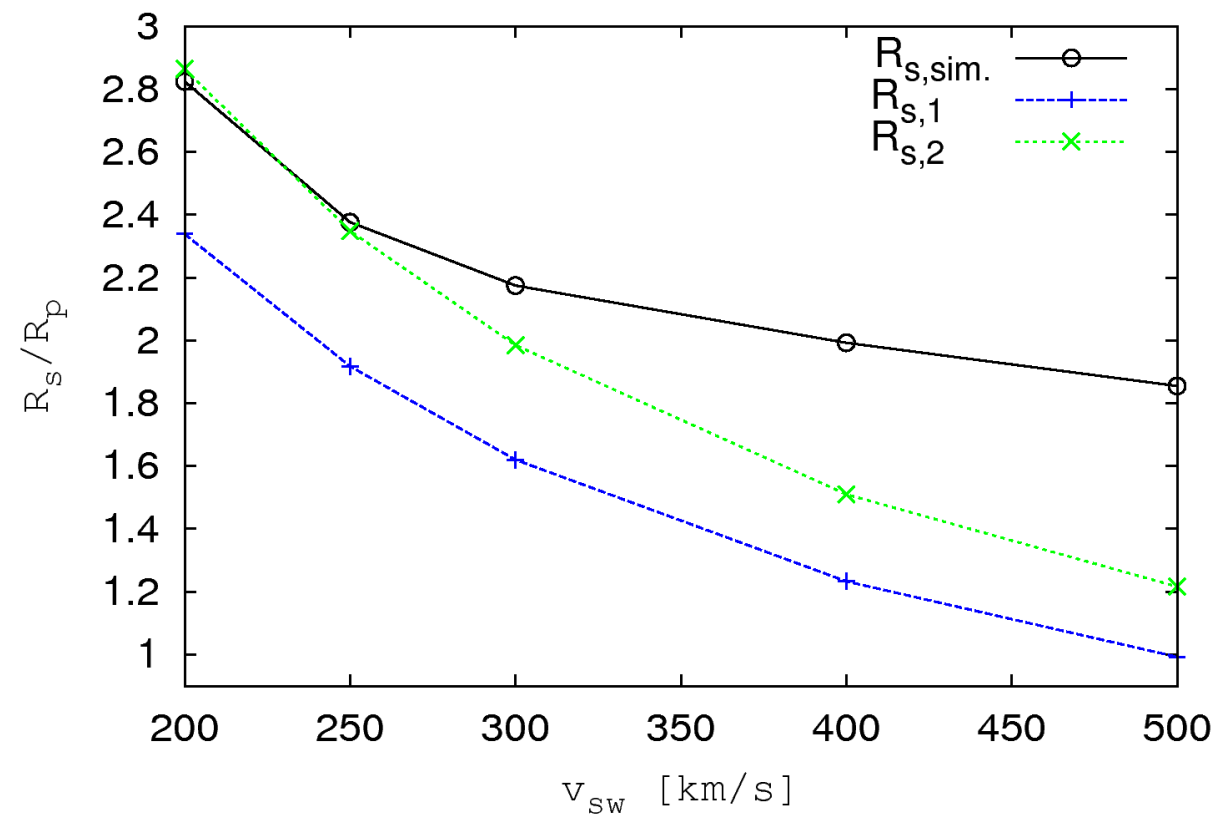

Figure 4.2: Standoff distance as a function of a) stellar wind density $n_{\mathrm{sw}}$, and b) stellar wind velocity $v_{\mathrm{sw}}$, derived from simulations and analytical expressions. Black solid line, $R_{\mathrm{s}, \mathrm{sim}}$ : standoff distance calculated from simulation runs. Blue dashed line, $R_{\mathrm{s}, 1}$ : standoff distance calculated using Eq. 4.3. Green dashed line, $R_{\mathrm{s}, 2}=\sqrt{C} R_{\mathrm{s}, 1}$ for $C=1.5$. 


\section{Quasiparallel stellar wind interaction}

\subsection{Introduction}

A stellar wind interaction as a whole is called perpendicular if the angle $\alpha_{\mathrm{sw}, 0}$ between the IMF and the effective stellar wind is $90^{\circ}$ and parallel if it is $0^{\circ}$. Similarly, if $\alpha_{\mathrm{sw}, 0}$ is only approximately $90^{\circ}$ or $0^{\circ}$ the stellar wind interaction is called quasiperpendicular or quasiparallel respectively. The word "approximately" is a key term here since these two types of interaction together fill, depending on convention, most or all of the range of angles between $0^{\circ}$ and $90^{\circ}$. The quasiperpendicular case is often regarded to cover a greater range of angles than the quasiparallel one but no clear dividing line exists between the two.

The magnetic field lines in the Solar System's ecliptic plane form spirals, known as Parker spirals. The reason for this configuration is that the magnetic field lines can be said to be frozen-in, i.e. following the infinitesimal plasma fluid elements as these move around (or vice versa) 1 . The magnetic field lines do on one hand reach out of the star and thus rotate with the plasma which makes up the star. On the other hand, the magnetic field lines also follow the stellar wind, moving more or less radially away from the central star. The result is magnetic field lines shaped almost like Archimedean spira $\left.\right|^{2}$ arms which can be perceived as both moving away from the star and rotating with the star, see Fig. 5.1. This spiral picture has to be understood as only a first approximation however. Reality is, as so often, a bit more more complicated. To begin with, the stellar wind is not completely radial since the plasma motion is influenced by the magnetic field and not only the other way around. We know from the Solar System that the solar wind speed varies with time within a factor of two, that there are interplanetary coronal mass ejections, shock waves propagating through the solar wind and turbulence altering the local field angle (see e.g. Schwenn and Marsch 1990, 1991, Borovsky and Funsten|2003). Also, the Sun itself does not have one well-defined rotation period due to differential rotation. More sophisticated

\footnotetext{
${ }^{1}$ Although the notion of frozen-in magnetic field lines is an incredibly useful concept, it is frequently not explained as rigorously as it should be. The correct meaning in the ideal case is: The string of infinitesimal plasma fluid elements which coincide with a particular magnetic field line at a particular point in time will coincide with a single magnetic field line at any other point in time. The only exception is topological changes, e.g. at reconnection. This is also the only sense in which one can identify magnetic field lines at different times with each other: we simply decide it is the same field line that coincides with same string of fluid elements at all times. The notion of moving field lines would be ambiguous without this convention.

${ }^{2}$ An Archimedean spiral is a spiral on the form $r=a+b \theta$, where $(r, \theta)$ are polar coordinates and $a, b$ are constants.
} 


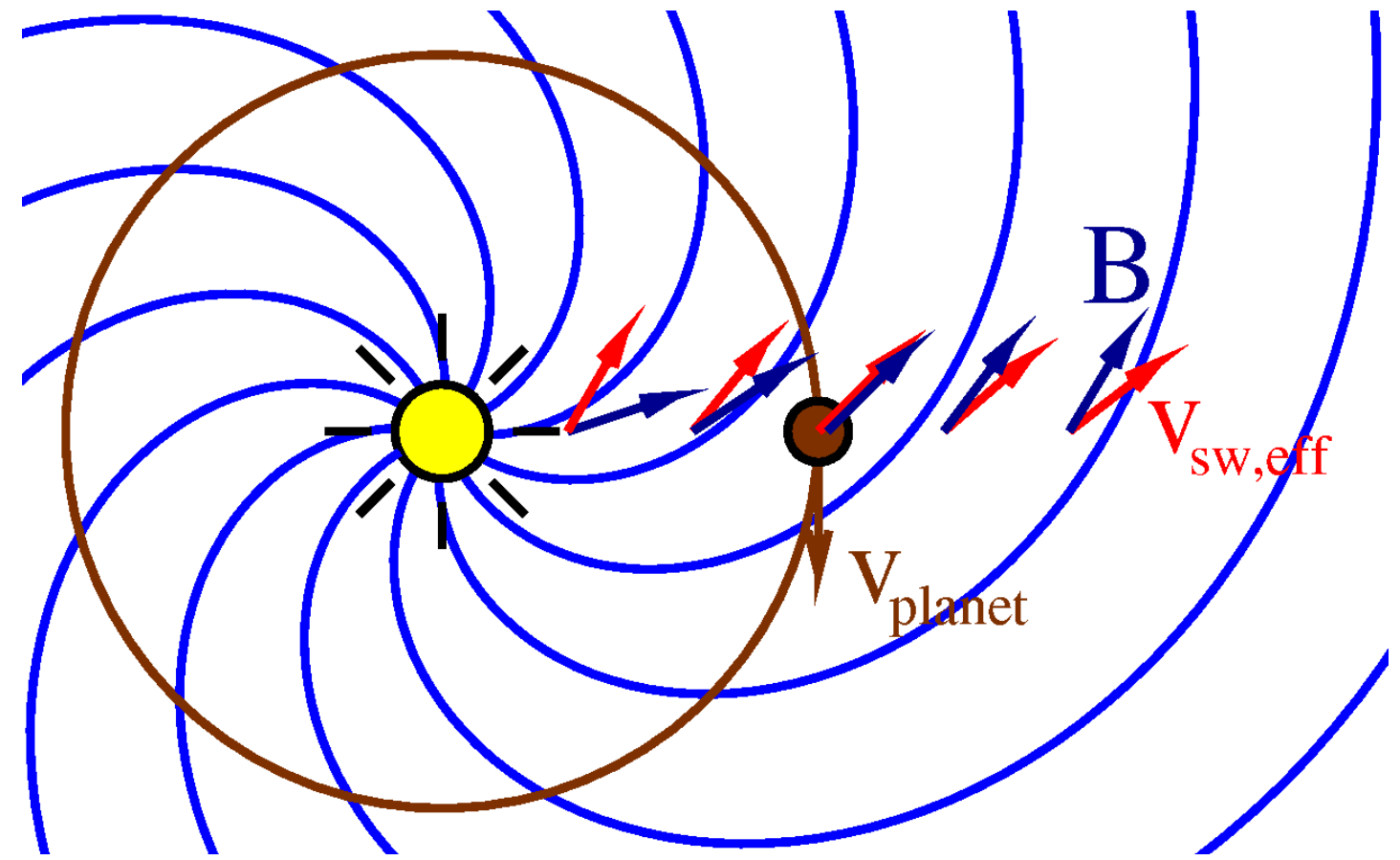

Figure 5.1: Illustration of how the magnetic field, $B$, and the effective stellar wind (i.e. stellar wind in the frame of the planet), $v_{\mathrm{sw}, \text { eff }}$, change direction for different orbital distances. $v_{\text {planet }}$ is orbital velocity of the planet. The planet is here located in an orbit where $B$ and $v_{\mathrm{sw}, \mathrm{eff}}$ are parallel.

models, incorporating the effect of magnetic fields on the motion of the stellar wind, can be found in e.g. Mariani and Neubauer (1990), Weber and Davis (1967).

It follows from the argument above that the direction of IMF changes with distance from the star: from almost radial on the star's surface, towards toroidal at great distances assuming the stellar wind speed does not increase too quickly with distance. The stellar wind direction in the frame of a planet in circular orbit depends on distance too due to the orbital motion of the planet. As the distance to the star increases the orbital velocity decreases and the stellar wind direction goes from almost toroidal toward radial, i.e. the opposite of the trend for the IMF. With this simple argument one can deduce that $\alpha_{\mathrm{sw}, 0}$ changes with orbital distance and that there has to be some orbital distance at which, at least ideally, both the IMF and the effective stellar wind are parallel, i.e. where one has a parallel stellar wind interaction.

We could in principle calculate the angle $\alpha_{\mathrm{sw}, 0}$ between stellar wind and IMF as a function of distance to find such orbits, but we take a shortcut instead since we are particularly interested in the case $\alpha_{\mathrm{sw}, 0}=0^{\circ}$. We observe that having the stellar wind velocity and the IMF in the local frame of reference be parallel is the same as staying on the same magnetic field line, connected to the same point on the star's surface. The Parker spiral field lines can, as previously mentioned, be perceived as both expanding away from the star and as rotating with the star. Thus, if we stay on a circular orbit in the ecliptic plane with an orbital period coinciding with the stellar rotation period we will have a parallel 
stellar wind. Thus the orbital distance of interest is determined by the condition

$$
\Omega_{\star} r=\sqrt{\frac{G M_{\star}}{r}}
$$

where $\Omega_{\star}$ and $M_{\star}$ are the stellar angular rotation velocity and stellar mass respectively. $G$ and $r$ are the gravitational constant and orbital distance from the star. Eq. 5.1 is easily rewritten as

$$
r=\left(G M_{\star}\right)^{1 / 3} \Omega_{\star}^{-2 / 3}
$$

which for ease of use can also be rephrased as

$$
r \approx 0.020 \times\left(\frac{M_{\star}}{M_{\odot}}\right)^{1 / 3}\left(\frac{T_{\star}}{1 \text { day }}\right)^{2 / 3} \mathrm{AU}
$$

where $M_{\odot}$ is the solar mass, $T_{\star}$ is the stellar rotation period and AU refers to astronomical units. Using solar values yields $r=0.17 \mathrm{AU}$, or about half of Mercury's perihelion at 0.31 AU. Note that the expression in Eq. 5.2 is conveniently independent of $v_{\mathrm{sw}, 0}$ and $B_{\mathrm{sw}, 0}$ and only assumes that the stellar wind is radial and that the stellar wind magnetic field is frozen-in. It does not assume that the stellar wind speed is constant.

The existence of such orbits is interesting since planets in them could potentially possess very different types of magnetospheres since many common features of stellar wind interaction with planets depend on the IMF having a component perpendicular to the stellar wind direction. Maybe the most obvious example is the draping of magnetic field which obviously requires a perpendicular component, but also pick-up requires it. Somewhat less obvious are the consequences for the bow shock.

Similar to stellar wind interaction, shocks can be classified as perpendicular, quasiperpendicular, parallel or quasi-parallel depending on the angle $\theta_{B n}$ between the upstream magnetic field and the shock surface normal, $\boldsymbol{n}$. Since the bow shock surrounding a planet is not a flat surface, the angle between the upstream magnetic field and the shock normal varies between different parts of the shock surface. Therefore one will often have a parallel shock somewhere on the bow shock even if the stellar wind interaction is not perfectly parallel. Thus, it is often not really a question of if the system has a parallel bow shock but where on the shock surface, and if it is close enough to the planet as to be relevant at all.

Parallel bow shocks are interesting because in theory, a parallel magnetic field is not affected by the shock. The magnetic field component perpendicular to a shock normal, i.e. parallel to the shock surface, is always enhanced together with the plasma density as it crosses the shock. This is a simple consequence of frozen-in magnetic fields. The parallel component on the other hand must stay constant due to the vanishing divergence of magnetic fields. In other words, if there is no perpendicular field component, then it can not be enhanced, or at least not in a stable shock. In reality, parallel shocks are sensitive to small deviations from a parallel magnetic field, i.e. they are unstable and always manage to produce a locally perpendicular field component even if no such exists farther upstream. They are also very oscillatory up to large distances in front of the shock, in the so called foreshock where the upstream plasma is first influenced by the shock's presence (Baumjohann and Treumann 1996). Hybrid simulations have also shown this to be true for high Mach numbers (Burgess 1989). 
It can be shown in a similar vein that parallel shocks should theoretically reduce to gasdynamic shocks within the realm of MHD but also this does not hold true in practice. Kinetic effects are important for quasi-parallel shocks (Treumann and Jaroschek 2008) and it should thus be more appropriate to investigate this problem with a hybrid code instead of MHD. With a quasiparallel stellar wind interaction we obtain a quasiparallel shock on the very dayside magnetosphere and can expect to destroy some of the strict division between undisturbed stellar wind and magnetosheath that we are so used to seeing. We will not go into the deeper theory of quasiparallel shocks but instead refer to Treumann and Jaroschek (2008) and its following parts.

Although quasiparallel shocks have been well-studied, especially in theoretical studies (see e.g. Filippychev 2000, and references therein), the same does not hold true for quasiparallel stellar wind interaction with planets as one could expect given the absence of it in the Solar System. We have therefore chosen to study quasiparallel stellar wind interaction with hybrid simulations by comparing a quasiparallel interaction $\left(\alpha_{\mathrm{sw}, 0}=10^{\circ}\right)$ with the corresponding perfectly perpendicular case $\left(\alpha_{\mathrm{sw}, 0}=90^{\circ}\right)$. Also included is an intermediate case $\left(\alpha_{\mathrm{sw}, 0}=30^{\circ}\right)$ in order to be able to study the transition better. These three simulation runs will be referred to as the perpendicular, the intermediate and the quasiparallel simulation run respectively. We have not used a perfectly parallel case because 1.) a truly parallel case will not last for very long in practice due to the always present fluctuations that we know exist in the solar wind, 2.) quasiparallel simulation runs have proven to reach stationary state much more slowly than others, and 3.) the simulated ionosphere has not always been able to prevent the stellar wind from penetrating through it for low values of $\alpha_{\mathrm{sw}, 0}$. We want to stay clear of this in order to avoid among other things chemical reactions between the stellar wind and atmosphere, something which is not incorporated into the simulation model.

Using a perfectly perpendicular case as reference is both useful and interesting not only because it is as far from a parallel case one can come but also because it can be shown that such a case should ideally be perfectly mirror symmetric. More specifically, it can be shown that the hybrid model equations have a certain mirror symmetry ${ }^{3}$ such that if we use mirror symmetric boundary conditions then we will obtain similarly mirror symmetric solutions to the hybrid model equations in section 2.1. Due to the orientation of the IMF we should therefore expect the perpendicular simulation run to have the polar plane, $y=0$, as a plane of symmetry meaning that the two halves of the simulation box $y>0$ and $y<0$ are mirror images of each other ${ }^{4}$. It is interesting to compare this with e.g. ideal MHD which obeys yet another mirror symmetry and for which both the polar and equatorial planes would be planes of symmetry if one used our boundary conditions. In a very similar vein we can also conclude that a perfectly parallel case should, theoretically, be cylindrically symmetric. Having said all this it should of course

\footnotetext{
${ }^{3}$ The type of mirror symmetry we are referring to is equivalent to the statement that Eqs. 2.9 and 2.10 are invariant under the transformation $x \rightarrow-x,\left(B_{y}, B_{z}\right) \rightarrow\left(-B_{y},-B_{z}\right), v_{p, x} \rightarrow-v_{p, x}$ and implicitly also under $u_{\mathrm{i}, x} \rightarrow-u_{\mathrm{i}, x}, E_{x} \rightarrow-E_{x}, \boldsymbol{j}_{x} \rightarrow-\boldsymbol{j}_{x}$. The same statement is of course true also for any other permutation of $x, y, z$. It could be argued that the type of the symmetry is technically not a mirror symmetry but it is very similar. The same symmetry can also be used to show that reversing the direction of the IMF leads to the same kind of stellar wind interaction with unmagnetized planets.

${ }^{4}$ The boundary conditions are mirror symmetric in the $z=0$ plane too of course, but in another sense in which Eqs. 2.9 and 2.10 are not mirror symmetric. We can therefore not expect the equatorial plane, $z=0$, to be yet another symmetry plane although it can be tempting to believe so.
} 
be mentioned that random (super)particle fluctuations, turbulence and instabilities may still break these symmetries, in particular for a perfectly parallel case.

To conclude, comparing the quasiparallel case with a perpendicular and an intermediate case allows us to separate the effects of changing the IMF angle from any peculiarities that might otherwise result from our particular choice simulation scenario. The simulation study described in this chapter will appear in Johansson et al. (2010).

\subsection{Model parameters}

Similar to the case of expanding atmospheres, we choose to work with a simulation scenario which in many ways approximates that of an Earth-like planet in low orbit around a Sun-like star. Our planet therefore has a radius $R_{\mathrm{p}}=R_{\text {Earth }}$ but still lacks an intrinsic magnetic field.

We have made three simulation runs, all of them identical except for the parameter we want to study, $\alpha_{\mathrm{sw}, 0}$, the angle between the IMF and the stellar wind velocity in the frame of the planet. We have used the values $\alpha_{\mathrm{sw}, 0}=90^{\circ}, \alpha_{\mathrm{sw}, 0}=30^{\circ}$ and $\alpha_{\mathrm{sw}, 0}=10^{\circ}$.

Our simulation box here has the dimensions $5 R_{\mathrm{p}} \times 6 R_{\mathrm{p}} \times 7 R_{\mathrm{p}}$ and is divided into $104 \times 128 \times 148$ approximately cube-shaped cells, each with a width of approximately $0.047 R_{\mathrm{p}}$. The planet is located $-0.5 R_{\mathrm{p}}$ in the $z$ direction from the center of the simulation box. The reason for the irregular size of the simulation box and the non-center location of the planet is to minimize the influence of artefacts on the boundaries.

The time step size is

$$
\Delta t=0.04\left(\omega_{\mathrm{g}, \mathrm{H}^{+}}\right)^{-1}
$$

where $\omega_{\mathrm{g}, \mathrm{H}^{+}}$is the typical gyration frequency for hydrogen ions, i.e. the background stellar wind value. The simulations have run for a time equivalent to an undisturbed stellar wind passing through the simulation box more than 50 times. The reason for this much higher number compared to in chapter 3 is that these simulation runs have proven to reach quasistationary state much more slowly, in particular the quasiparallel run.

\subsubsection{Stellar wind parameters}

We choose to work with stellar wind parameters similar to those at a distance of $r=$ 0.2 AU from the Sun and thus calculate the corresponding stellar wind parameters using the methods already described in section 2.4. This is close to $r=0.17$ AU where a planet in circular orbit around the Sun would (ideally) be exposed to a parallel stellar wind, as has already been mentioned in connection with Eq. 5.3 .

As mentioned before, we are in practice forced to work in a low magnetic field limit due to the inherent constraints of hybrid simulations. We have however been able to relax this constraint for the work in this chapter, partly because we can here work with a smaller simulation box (in physical units) and partly because we here had the advantage of being able to use the faster AIKEF code as opposed to for the expanding ionosphere simulations. This is the reason why we use a stronger IMF in this set of simulations compared to in chapter 3, despite the fact that we are otherwise trying to model an interaction at a greater orbital distance where the IMF should be weaker.

The exact parameter values we have used are summarized in Table 5.1 . 


\begin{tabular}{|c|c|c|}
\hline \multicolumn{2}{|l|}{ Parameter } & Value \\
\hline Velocity & $v_{\mathrm{sw}, 0}$ & $330 \mathrm{~km} \mathrm{~s}^{-1}$ \\
\hline Number density & $n_{\mathrm{sw}, 0}$ & $210 \mathrm{~cm}^{-3}$ \\
\hline IMF/magnetic field & $B_{\mathrm{sw}, 0}$ & $12 \mathrm{nT}$ \\
\hline Velocity-IMF angle, run 1 & $\alpha_{\mathrm{sw}, 0}$ & $90^{\circ}$ \\
\hline run 2 & $\alpha_{\mathrm{sw}, 0}$ & $30^{\circ}$ \\
\hline run 3 & $\alpha_{\mathrm{sw}, 0}$ & $10^{\circ}$ \\
\hline Ion temperature & $T_{\mathrm{sw}, \mathrm{i}, 0}$ & $154500 \mathrm{~K}$ \\
\hline Electron temperature & $T_{\mathrm{sw}, \mathrm{e}, 0}$ & $309000 \mathrm{~K}$ \\
\hline Alfvénic Mach number & $M_{\mathrm{A}}$ & 18 \\
\hline Magnetosonic Mach number & $M_{\mathrm{ms}}$ & 3.7 \\
\hline
\end{tabular}

Table 5.1: Stellar wind parameters used in the simulation runs of this chapter.

\begin{tabular}{lcc}
\multicolumn{1}{c}{ Parameter } & & Value \\
\hline \hline Initial ion temperature & $T_{\mathrm{i}}$ & $3000 \mathrm{~K}$ \\
Initial electron temperature & $T_{\mathrm{e}}$ & $3000 \mathrm{~K}$ \\
Absorption cross section & $\sigma_{\mathrm{a}}$ & $6.55 \cdot 10^{-22} \mathrm{~m}^{2}$ \\
Ionization cross section & $\sigma_{\mathrm{i}}$ & $6.51 \cdot 10^{-22} \mathrm{~m}^{2}$ \\
Ionization rate at infinity & $I_{\infty} \sigma_{\mathrm{i}}$ & $6.29 \cdot 10^{-6} \mathrm{~s}^{-1}$ \\
Total production rate & $Q_{\mathrm{O}^{+}, \text {tot }}$ & $2.81 \cdot 10^{29} \mathrm{~s}^{-1}$ \\
Neutral oxygen scale height & $H$ & $92.8 \mathrm{~km}$ \\
Neutral oxygen temperature & $T_{\mathrm{O}}$ & $1764 \mathrm{~K}$ \\
Neutral oxygen boundary density & $n_{\mathrm{O} \text {, surf. }}$ & $5.00 \cdot 10^{13} \mathrm{~m}^{-3}$ \\
\hline \hline
\end{tabular}

Table 5.2: Ionospheric parameters used in the simulation runs of this chapter.

\subsubsection{Ionospheric parameters}

To derive the parameters we need we use a simple model of a neutral atmosphere, inspired by the atomic oxygen-dominated upper ionosphere of Venus. We then assume that this neutral atmosphere generates oxygen ions through photoionization.

We only model the atmosphere above a certain altitude/density and assume that ions which motion take them below this altitude are "recombined" in the denser lower atmosphere, i.e. are removed from the simulation. No ions are assumed to emerge from the atmosphere below this altitude. This way we can ignore what the atmosphere looks like farther below and make this altitude identical to the planetary boundary in the simulation box as described in section 2.3 .

The neutral atomic oxygen atmosphere we use is an ordinary hydrostatic number density profile

$$
n_{\mathrm{O}}(z)=n_{\mathrm{O}, \text { surf. }} e^{-z / H}
$$

where $n_{\mathrm{O} \text {, surf. }}=5 \cdot 10^{13} \mathrm{~m}^{-3}$ is the density at the lower absorbing boundary, $z$ is the altitude above the lower absorbing boundary and $H$ is a constant scale height

$$
H=\frac{k_{\mathrm{B}} T_{\mathrm{O}}}{16 m_{\mathrm{p}} g} \approx 93 \mathrm{~km}=0.015 R_{\mathrm{p}}
$$




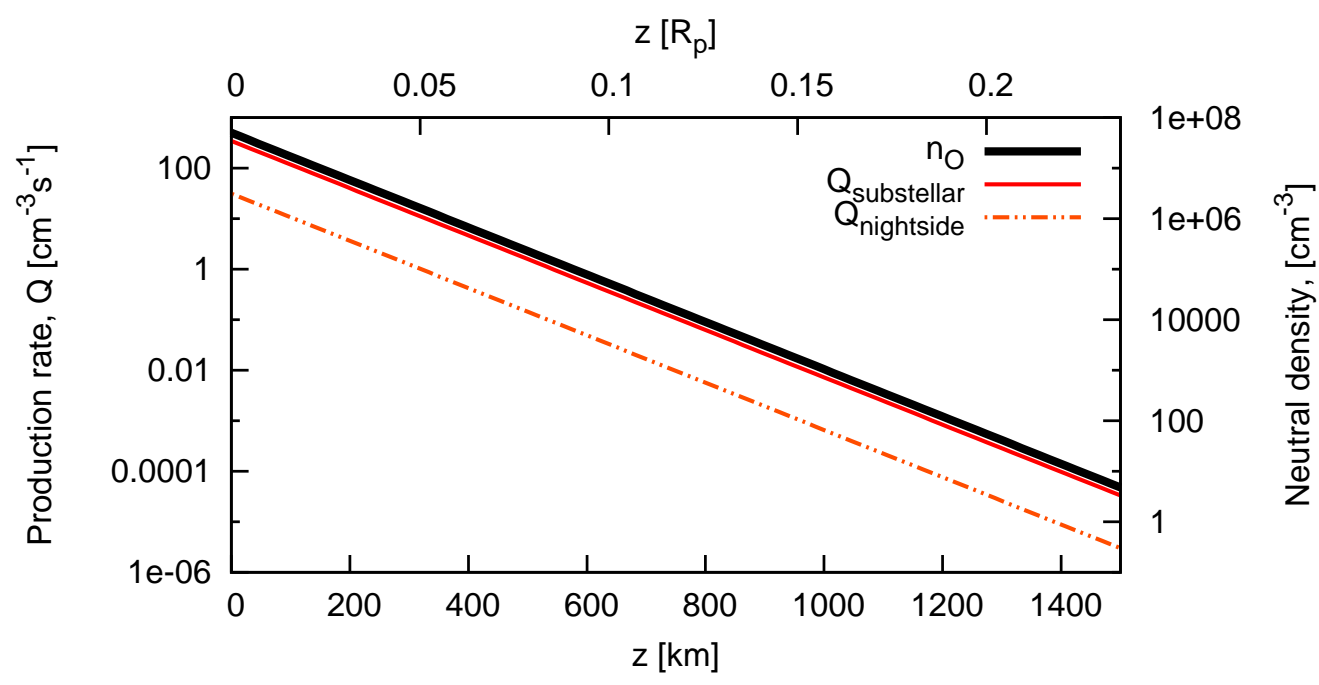

Figure 5.2: Neutral oxygen density and oxygen ion production rate used for simulation runs. Thick solid black line $\left(n_{\mathrm{O}}\right)$ : Neutral oxygen density. Red solid line $\left(Q_{\text {substellar }}\right)$ : Oxygen ion production rate over the substellar point. Red dash-dot-dotted line $\left(Q_{\text {nightside }}\right)$ : Oxygen ion production rate for the nightside.

calculated for a neutral oxygen temperature of $T_{\mathrm{O}}=1764 \mathrm{~K}$ and a gravitational acceleration $g=9.81 \mathrm{~m} \mathrm{~s}^{-2}$ equal to that of the Earth. $m_{\mathrm{p}}$ is the proton mass and $k_{\mathrm{B}}$ is the Boltzmann constant.

Using a hydrostatic profile like Eq. 5.5 which is usually motivated using the notion of the neutral atmosphere being a collisional fluid could at first sight be seen as suspect since we are only modeling the very upper part of the atmosphere, including the more or less collisionless exosphere. Therefore it is worth pointing out that also an exosphere like the one described in Schunk and Nagy (2000) approximates Eq. 5.5 in the limit of low scale heights $\left(H \ll R_{\mathrm{p}}\right)$ and low altitudes above the exobase ${ }^{5}\left(z \ll R_{\mathrm{p}}\right)$. Exospheres indeed do often have very different temperatures compared to the collisional atmosphere below and may have components with different temperatures but we do not want to complicate the model and do not want to have more arbitrarily set parameters than necessary. Therefore we settle on using the same temperature and scale height for the entire atmospheric profile. The atmospheric temperature we use may seem high but it is not much higher than the temperature of the upper ionosphere and exosphere of Earth, 700-1100 K (Schunk and Nagy 2000), or the exosphere of Venus, 200 - $1000 \mathrm{~K}$ (Chamberlain and Hunten 1987). We should also recognize that we are considering a planet at a distance of only $r=0.2 \mathrm{AU}$ from a Sun-like star where more energy is available to heat the upper atmosphere.

We use a standard Chapman layer for the ionospheric production rate, derived in the standard way as described in e.g. Schunk and Nagy (2000) and based on the neutral profile in Eq. 5.5. For simplicity we use only one absorption cross section $\sigma_{\mathrm{a}}$, one photoionization cross section $\sigma_{\mathrm{i}}$ and one photon flux $I_{\infty}$ for all wavelengths $50-950 \AA$ Richards

${ }^{5}$ The exospheric model described in $\mid$ Schunk and Nagy $\mid(2000)$ assumes that the exosphere is spherically symmetric and is populated by atoms on ballistic trajectories, i.e. completely collisionless. The exosphere has a lower boundary surface ("exobase") at which atoms are ejected with a Gaussian velocity distribution. 
et al. 1994) to calculate the photoionization rate. We calculate the cross sections by taking the photon flux-weighted averages using the EUVAC Solar Flux Model (Schunk and Nagy 2000, Richards et al. 1994). The photon flux we use is equivalent to twice that at a distance of $r=0.2 \mathrm{AU}$ from the Sun. We introduce this extra factor of two to make sure that we stay in a regime where the stellar wind never penetrates too deeply into the atmosphere. It is our experience that this often happens for quasiparallel and parallel simulation runs. While this extra factor is arbitrarily chosen it is worth pointing out that the end effect is about the same or less compared to had we changed from a quiet Sun to an active Sun (Huebner et al. 1992). The extra factor can in principle also be interpreted as using a somewhat younger Sun since the photoionization rate of Sun-like stars also decrease considerably over their lifetimes (Ribas et al. 2005).

The resulting ionospheric $\mathrm{O}^{+}$production rate is

$$
Q_{\mathrm{O}^{+}, \text {Chapman }}(z, \theta)=\left(n_{\mathrm{O}, \text { surf. }} e^{-z / H}\right) \sigma_{\mathrm{i}} I_{\infty} \exp \left(-\frac{\sigma_{\mathrm{a}} n_{\mathrm{O}, \text { surf. }} H}{\cos \theta} e^{-z / H}\right)
$$

where $\theta$ is the angle of incidence of the incoming EUV radiation 6 . We use this production rate for the dayside. To avoid singularities at the terminator plane $\left(\theta=90^{\circ}\right)$ we will in practice only use it for $0 \leq \theta<87^{\circ}$.

We also add, somewhat arbitrarily, a small background production to accommodate for the fact that there are other in principle other ion production mechanisms than photoionization and because the hybrid model numerically requires the plasma density not to be too low. A nightside production rate alleviates this problem by helping to fill the simulation box on the nightside. This added background production rate is set to be equivalent to $10 \%$ of the dayside production rate at an angle of $\theta=30^{\circ}$, i.e.

$$
Q_{\mathrm{O}^{+}, \text {background }}=0.10 \cdot Q_{\mathrm{O}^{+}, \mathrm{Chapman}}\left(z, 30^{\circ}\right) .
$$

The ionospheric plasma itself is produced with an initial ion and electron temperature of $T_{\mathrm{i}}=T_{\mathrm{e}}=3000 \mathrm{~K}$. These numbers too are based on Venus values (Schunk and Nagy 2000). The neutral oxygen density together with the substellar and nightside production rates are plotted in Fig. 5.2 .

We will for future reference calculate the characteristic gyration scale of picked up ionospheric oxygen ions given the stellar wind in the preceding section.

$$
r_{\mathrm{g}, \mathrm{O}^{+}}=\frac{16 m_{\mathrm{p}} v_{\mathrm{sw}, 0}}{e B_{\mathrm{sw}, 0}} \approx 0.72 R_{\mathrm{p}}=16 \text { cells }
$$

Since this value is comparable to the planet size, it may at first sight seem to imply that pickup should be present but as we shall see, things are little bit more complicated than that. 

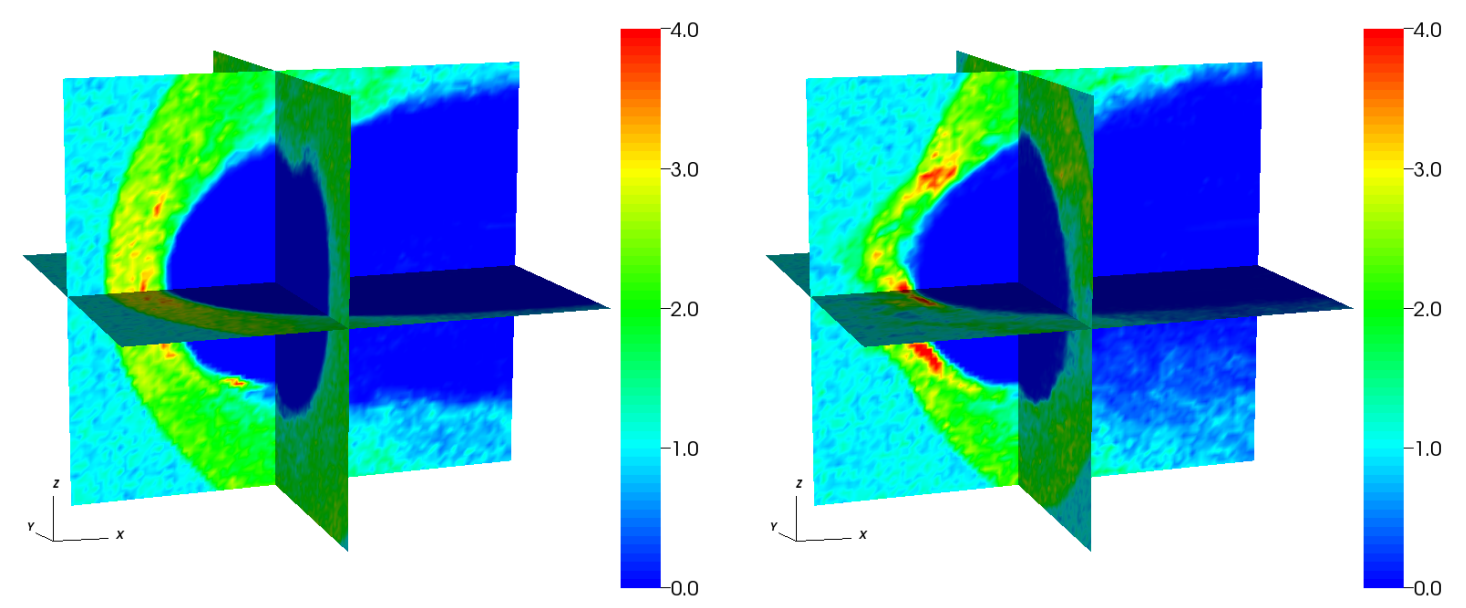

Figure 5.3: Three-dimensional overviews of simulation results for the stellar wind density in the form of intersecting cross sections for the perpendicular (left) and quasiparallel (right) simulation run. The density is normalized to the background stellar wind density. Parts of the simulation box have been removed to give a better overview.

\subsection{Results}

The simulation results can be viewed in Figs. 5.3, 5.4, 5.5, 5.6, 5.7, 5.8 and 5.9, Fig. 5.3 shows a three-dimensional overview of the stellar wind density for the perpendicular run and the quasiparallel run. The other figures depict various physical quantities on simulation box cross sections. We use a layout similar to in chapter 3 , i.e. figures are organized such that every column of smaller figures represents one simulation run and every row represents the same physical quantity on the same cross section. Color scales are kept the same for all figures depicting the same physical quantity to make comparisons easier. The arrows, representing vectors projected on the particular cross sections, are however not in the same scale in different figures and the lengths are therefore not directly comparable. The arrow lengths themselves are proportional to the square root of the projected vector magnitude in order to reduce the span of arrow lengths within the same figure, something which otherwise often makes vector plots unclear. For vector plots, the color represents the true magnitude of the three-dimensional vector.

We begin by looking at our textbook case, the perpendicular simulation run $\left(\alpha_{\mathrm{sw}, 0}=\right.$ $90^{\circ}$ ) in Figs. $5.4 \mathrm{dgj}$ and $5.5 \mathrm{dgj}$ and note that as expected it very much follows the general interaction picture we described also for our stationary simulation run in section 3.3 . We have a bow shock upstream of which the stellar wind is unaffected by the existence of the obstacle. Downstream of the bow shock is the magnetosheath, characterized by its slower and more dense stellar wind and consequently enhanced magnetic field. Downstream of the magnetosheath is in turn the ionosphere, the planet itself and the tail of ionospheric plasma escaping the planet.

The perpendicular run is as predicted symmetric in the equatorial plane, Figs. 5.4 adgj

\footnotetext{
${ }^{6}$ This profile is in practice more sophisticated than necessary since the upper part of the ionosphere we are interested in is more or less optically thin. Thus Eq. 5.7 is in practice quite well approximated by setting the last factor to one.
} 
and $5.6 \mathrm{~d}$, and asymmetric to various degrees in the polar plane, Figs. $5.5 \mathrm{dg} \mathrm{dg}$ and $5.7 \mathrm{ad}$. The mirror symmetry is almost perfect but one can note some small signs that it is not. For example, if the magnetic field lines were perfectly mirror symmetric, then the magnetic field would have zero $x$ and $z$ components in the entire polar plane but Fig. 5.5a reveals that it is not so.

Note also how the magnetic draping is much stronger on the negative $z$ hemisphere compared to the positive $z$ hemisphere and that the ionosphere and the ionospheric tail have a preference for positive $z$. Incidentally, we have seen the same tendency for the displacement of the draping but much weaker in Fig. 3.4a in the stationary ionosphere run in section 3.3 .

Having familiarized ourselves with the perpendicular run can start looking at what happens when we reduce the IMF angle $\alpha_{\mathrm{sw}, 0}$. We begin by looking at the bow shock in the equatorial plane, Figs. 5.4a-f and 5.6 $\mathrm{a}-\mathrm{c}$. At first in the perpendicular run, the bow shock is clearly visible as a surface of sudden change of stellar wind density and velocity but also as a sudden enhancement of the magnetic field component parallel to the shock surface. As we have mentioned, theory predicts that whereas a quasiperpendicular shock is made up of a thin, quasistationary shock surface, a parallel or quasiparallel shock neither has a well-defined thickness, nor is it very stationary. This fits well with how a section of "fuzzy", quasiparallel shock wanders from infinite negative $y$ to the dayside shock surface as we move from the perpendicular to the quasiparallel run. Obviously, given the size of this section of the shock surface, it is not only the exact parallel shock that causes the otherwise so discrete shock surface to break up. A crude estimate from Fig. 5.4 gives that shock angles up to at least $\theta_{B n} \sim 15^{\circ}$ show this behavior and could be counted as quasiparallel. That is why the quasiparallel shock in the quasiparallel run can be seen also in the polar plane in e.g. Fig. 5.5f.

A closer look at the quasiparallel run in the stellar wind density and velocity in Figs. 5.4f, 5.6, 5.5f and 5.7k reveals that we can see a vaguely defined foreshock upstream of where the "missing" shock surface in the quasiparallel run is located. Although somewhat hard to see from the figures, this is visible as the stellar wind density and velocity being somewhat perturbed upstream of the obvious part of the parallel shock, almost up to the upstream boundary of the simulation box judging from Fig. 5.6. A Apart from the creation of a foreshock, we can also observe how the substellar magnetosheath has transformed into being more heterogeneous with scattered patches of high density just above the ICB and no longer has a very well-defined boundary to the upstream stellar wind. Fig. 5.4 shows how some scattered oxygen ions have actually managed to travel into the aforementioned foreshock region. It is known that fast downstream particles can indeed gyrate along the field lines upstream of a shock as long as they are fast enough and are not perturbed. Why we should have such fast oxygen ions is uncertain however.

Turning our attention to the draping of the magnetic field in Figs. $5.4 \mathrm{a}-\mathrm{c}$ and $5.5 \mathrm{a}-\mathrm{c}$ we see, as anticipated, that the magnetic field pile-up on the dayside decreases as the perpendicular IMF component, $B_{y}=B_{\mathrm{sw}, 0} \sin \alpha_{\mathrm{sw}, 0}$, decreases. In fact, the maximum magnetic field value in Figs. $5.5 \mathrm{a}-\mathrm{c}$ stays almost perfectly proportional to $\sin \alpha_{\mathrm{sw}, 0}$ if one compares the three runs.

Here we can also see another consequence of draping in combination with the decreased IMF angle $\alpha_{\mathrm{sw}, 0}$ : Draping itself means that the field lines "drape" around the planet as they follow the stellar wind flow around the planet. However, the draped field 
lines also have to connect to the IMF at infinity which adds another two bends to the field lines. When the IMF angle decreases, one of these extra bends is straightened out while the other one transforms into a sharper and sharper curve. The end effect is that field lines are forced into a more and more extreme " $\mathrm{S}$ " shape in the equatorial plane. The reason we mention this is because the extra, sharpening field line bend should, with a decreasing IMF angle, sooner or later lead to something which resembles a second current sheet. Indeed, that is what we see in the intermediate and quasiparallel runs, Figs. 5.4 pc, on the negative $y$ side of the planet as regions of very weak magnetic field separating areas with magnetic field in the opposite directions as well as in the terminator plane in Fig. $5.8 \mathrm{c}$ for the quasiparallel simulation run.

We can also note that the strength of the overall electric field strength decreases as $\alpha_{\mathrm{sw}, 0}$ decreases, as can be seen in Figs. $5.6 \mathrm{~d}-\mathrm{f}$ and $5.7 \mathrm{~d}-\mathrm{f}$. This is of course very predictable since it is a convective electric field which is a function of the stellar wind flow and magnetic field through

$$
\boldsymbol{E}=-\boldsymbol{v} \times \boldsymbol{B}
$$

which in turn means it depends on the angle between the total plasma velocity $\boldsymbol{v}$ and the magnetic field. A little oddity in this context is the peculiar effect that when the IMF angle decreases, the electric field fluctuations around zero in the equatorial plane tend to become perpendicular to the stellar wind flow as can be seen in Figs. 5.6d-f. The explanation is simple if we add small fluctuations $\delta \boldsymbol{v}, \delta \boldsymbol{B}$ and $\delta \boldsymbol{E}$ to constant background fields $\boldsymbol{v}_{0}, \boldsymbol{B}_{0}$ and $\boldsymbol{E}_{0}$

$$
\begin{aligned}
\boldsymbol{B} & =\boldsymbol{B}_{0}+\delta \boldsymbol{B} \\
\boldsymbol{E} & =\boldsymbol{E}_{0}+\delta \boldsymbol{E} \\
\boldsymbol{v} & =\boldsymbol{v}_{0}+\delta \boldsymbol{v}
\end{aligned}
$$

and assume that also the constant background terms satisfy Eq. 5.10. Then we obtain

$$
\delta \boldsymbol{E} \approx-\boldsymbol{v}_{0} \times \delta \boldsymbol{B}-\delta \boldsymbol{v} \times \boldsymbol{B}_{0}
$$

which implies that $\delta \boldsymbol{E}$ has to be perpendicular to $\boldsymbol{v}_{0}$ in the limit of $\alpha_{\mathrm{sw}, 0} \rightarrow 0$ where $\boldsymbol{B}_{0}$ and $\boldsymbol{v}_{0}$ are parallel. It is this tendency we see in Fig. 5.6f.

In the equatorial plane, Figs. $5.4 \mathrm{~d}-\mathrm{i}$, we see in the respective plasma densities how the entire dayside ICB in the equatorial plane seems to more or less follow the draped magnetic fields. As the draping then decreases with $\alpha_{\mathrm{sw}, 0}$, the ICB and the ionosphere retreat somewhat closer to the planet around the terminator. The overall ICB also becomes less well-defined, especially on the tail side as the draped magnetic field weakens and is unable to keep the two species separate. Although it may be hard to see from Figs. 5.4f and 5.5F, the dayside stellar wind almost starts to touch the planet surface (i.e. the ionabsorbing boundary) in the quasiparallel run, in particular on the negative $z$ hemisphere.

Given the previously calculated gyration length scale of $r_{\mathrm{g}, \mathrm{O}^{+}} \sim 0.72 R_{\mathrm{p}}$ from Eq. 5.9 we should naively expect pick-up, i.e. oxygen being accelerated away from the ICB on the negative $z$ side of the planet by the convective electric field. Looking at the oxygen density and velocity in Figs. 5.5g-1 we can only see something that looks like traces of pick-up. In fact, the ionosphere is actually thinner on the negative $z$ side than on the positive $z$ side, opposite to what we would expect with pick-up (cf. e.g. Boesswetter et al. 2004, Simon et al. 2006). 
We begin with the weak pick-up and the perpendicular simulation run. As we can see in Figs. $5.5 \mathrm{ag}$, the very strong magnetic pile-up has diffused into the ionosphere. Since the draping is also very asymmetric being stronger on the negative $z$ side it has led to the local ionosphere being wrapped inside a layer of very enhanced magnetic field, about a factor $\sim 20$ stronger. This implies that oxygen ions within this layer which are initially accelerated away from the planet by the convected electric field, Fig. 5.7d, are in practice not that likely to gyrate very far away since the local gyration scale is a factor $\sim 20$ smaller, or $r_{\mathrm{g}, \mathrm{O}^{+}} \sim 0.035 R_{\mathrm{p}}$ which is smaller than the thickness of the layer itself $f^{7}, 0.2 R_{\mathrm{p}}$.

The above argument tacitly requires the bulk of ionospheric production to take place sufficiently deep within this layer of locally enhanced magnetic field. The ionospheric oxygen production rate, as illustrated in Fig. 5.2 does in practice decrease approximately exponentially with altitude having a scale height of only $H=0.015 R_{\mathrm{p}}=93 \mathrm{~km}($ Eq. 5.6$)$ equal to that of the background neutral atmosphere. Our ionosphere also does not at all have any additional "hot population" exosphere producing ions on greater altitude scales like e.g. $Q \propto \ln \left(R_{\mathrm{p}} / r\right)$ in Boesswetter et al. (2004), Simon et al. (2006). This means that if the draped layer of enhanced magnetic field that locally covers the planetary surface has a thickness of $\sim 0.2 R_{\mathrm{p}} \sim 14 \mathrm{H}$ then the production rate outside this draping is very, very small; only a fraction $\sim e^{-0.2 R_{\mathrm{p}} / H} \approx 10^{-6}$ of the production rate at the "surface" (the ionabsorbing planetary boundary). Since the magnetic enhancement decreases the farther downstream we go, the possibility of pick-up should increase which does seem to fit with the traces of pick-up we see in Figs. $5.5 \mathrm{gj}$ and that do seem to originate behind the terminator alone. One should also remember that our ionospheric production is weaker on the nightside than on the dayside, see Eqs. 5.7 and 5.8.

So far we have only referred to pick-up for the perpendicular simulation run. When the IMF angle decreases, the piled-up magnetic field weakens as $B \sim \sin \alpha_{\mathrm{sw}, 0}$ and the layer of magnetic enhancement gets thinner, which should imply that the ionosphere on the negative $z$ side again becomes more sensitive to pick-up through the stellar wind outside the enhanced region. Note however that although the influence of pile-up decreases, the gyration scale for pick-up outside the magnetically enhanced region decreases too, due to the IMF angle. This trend of increasing pick-up should be roughly what we see if we look at the pick-up in Figs. 5.5g-1 again, although admittedly the trend is not perfect considering that the intermediate run, Figs. $5.5 \mathrm{hk}$, has the lowest visible pick-up and even the greatest pick-up effect is still very weak.

The decreasing IMF angle also complicates the picture for the trajectories of picked up ions. For the perpendicular run, a picked-up ion should approximately follow a cycloid in the same approximate polar plane, but for a non-perpendicular IMF the oxygen will gyrate out of the polar plane, in our case in the negative $y$ direction. Looking at the densities and velocities in the terminator cross sections, Figs. 5.8d-i it is obvious that it is only in the quasiparallel simulation run where we can see pick-up, or more correctly, pick-up ions originating from and upstream of the terminator. We can also see how the pick-up ions gyrate in the negative $y$ direction, which explains where some of the scattered oxygen ions in the equatorial plane in Fig. 5.4 come from.

Separate from the question of pick-up itself we have previously observed how the ionosphere in all three simulation runs is strangely thin on the negative $z$ side compared

\footnotetext{
${ }^{7}$ In principle one should also consider that the shocked stellar wind velocity is lower by a factor of $\sim 2$
} 
to the opposite side of the planet in Figs. 5.5g-i. The terminator plane cross sections in Figs. 5.8d-f now reveal an interesting feature in the same region: The thin ionosphere on the negative $z$ side is in reality very local and very similar in all three simulation runs. We do not have any fully satisfactory explanation to why we have this locally depressed ionosphere compared to the positive $z$ side, or for that matter why the ionosphere on the positive $z$ side changes from a (smaller) depression to a bulge in the terminator cross sections.

One hint to the origin of the negative $z$ depression, should be the location. Again, this location is special since the magnetic field is stronger there due to pile-up and one may suspect that the curvature of the field lines is strong there as well due to the draping.

One hypothesis is that the magnetic field in this region gives rise to some kind of drift motion which transports oxygen ions toward the surface to be absorbed, preventing any substantial local ionosphere from being built up. The two important assumptions when reasoning in terms of drift motion are 1.) that the particle gyrations are smaller than some characteristic scale depending on the type of drift, and 2.) that the drifting particles do not significantly influence the field that gives rise to the drift motion, i.e. it is imposed from outside somehow. Actual tracing of ionospheric superparticles in the simulation box (not illustrated) does however show that most oxygen ions in the depressed ionosphere on the negative $z$ side of the planet do gyrate toward the surface.

Having this in mind we can continue and observe that if the piled-up magnetic field has diffused into the oxygen dominated ionosphere, then possibly it could be regarded as imposed on the ionosphere from outside and therefore relatively independent of what happens inside the ionosphere. If we are inside the oxygen dominated ionosphere there should be no pick-up (no stellar wind, no significant convective electric field) and the relevant typical oxygen gyration velocities should be thermal $\left(\sim 2.2 \mathrm{~km} \mathrm{~s}^{-1}\right)$ and therefore the gyration radii very small. Closer investigation of gradient drift and curvature drift for our simulation runs does however find that this is unlikely to be the mechanism. See appendix B.

Apart from magnetic drifts, there is of course also the $\boldsymbol{E} \times \boldsymbol{B}$ drift

$$
\boldsymbol{v}_{E}=\frac{\boldsymbol{E} \times \boldsymbol{B}}{B^{2}} .
$$

In principle, pick-up is also a form of $\boldsymbol{E} \times \boldsymbol{B}$ drift but on larger scales than we consider here. This drift is not something we would intuitively expect to be relevant in regions dominated by ionospheric oxygen, like close to the surface, since any significant convective electric field should reasonably not be generated by the hypothesized drift motion of oxygen itself but rather by the motion of the stellar wind. However, on the one hand, Figs. 5.5g-i and $5.8 \mathrm{~d}-\mathrm{f}$ do reveal that the locally depressed ionosphere on the negative $z$ side actually only reaches densities of $\sim 1-10 n_{\mathrm{sw}, 0}$ so it is not completely clear that is should be regarded as completely oxygen dominated. On the other hand, it is not at all obvious if the gyration radii are small enough, but it is possible if one first considers the locally smaller pick-up gyration scale we calculated before, $r_{\mathrm{g}, \mathrm{O}^{+}} \sim 0.035 R_{\mathrm{p}}$, and then in addition considers a locally slower stellar wind due to mixing with the ionosphere if we are sufficiently close to the surface. Despite these many uncertainties, we have plotted the $z$ component of the $\boldsymbol{E} \times \boldsymbol{B}$ drift velocity in Fig. 5.9 to at least test the idea that $\boldsymbol{E} \times \boldsymbol{B}$ drift may be involved. Note that the figures have their color scales intentionally saturated to make it easier to see the sign of the drift in the $z$ direction. 
We can see a positive $z$ component at about the same places where the ionosphere is thinnest in Figs. 5.5 g-i and 5.8 d-f but the agreement is not perfect. The relevant drift velocities in the $z$ direction are on the order of $\sim 1-10 \mathrm{~km} \mathrm{~s}^{-1}$. Similar to for magnetic drift, the fit seems to be best for the perpendicular run and less so when decreasing the IMF angle but no corresponding trend can be spotted in the actual oxygen density. We also do not really know why $\boldsymbol{E} \times \boldsymbol{B}$ should have a positive $z$ component at all from our basic understanding of the orientation of the electric and magnetic fields. Although an interesting hypothesis we are not yet ready to regard this as a truly convincing explanation.

Not having found any reasonable explanation for why the ionosphere is so thin on the middle of the negative $z$ side of the planet we must also consider the possibility of numerical error, that e.g. the strong magnetic field curvature in this region is simply too strong for the cell grid resolution in the simulation box and it is also possible that this gives rise to unphysical drifts. 
a) $\boldsymbol{B} / B_{\mathrm{sw}, 0}$

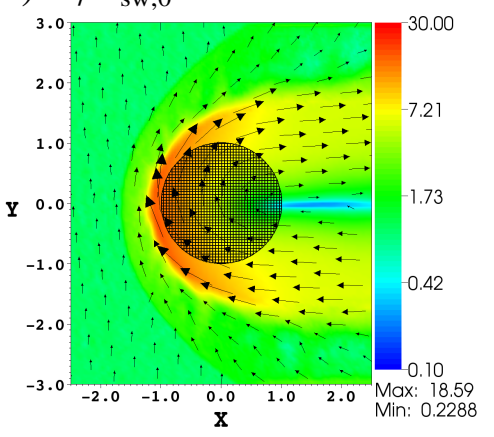

d) $n_{\mathrm{sw}} / n_{\mathrm{sw}, 0}$

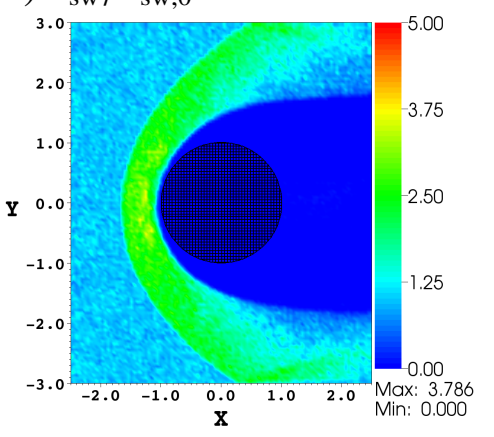

g) $n_{\mathrm{is}} / n_{\mathrm{sw}, 0}$

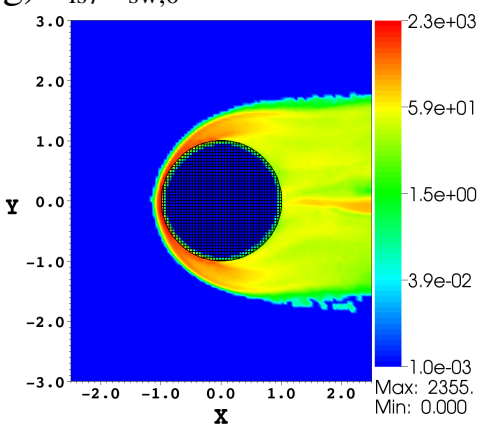

j) $v_{\text {is }} / v_{\mathrm{sw}, 0}$

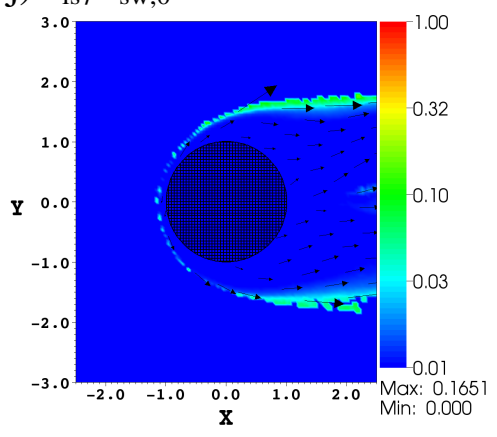

b) $\boldsymbol{B} / B_{\mathrm{sw}, 0}$

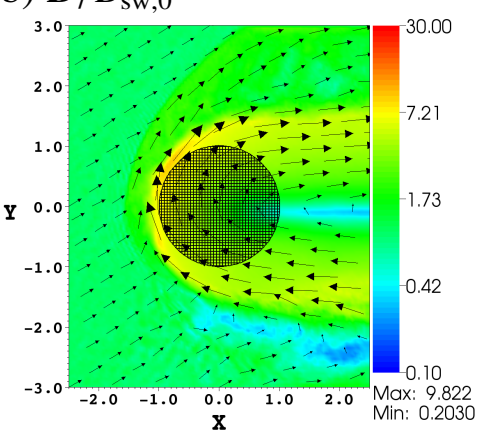

e) $n_{\mathrm{sw}} / n_{\mathrm{sw}, 0}$

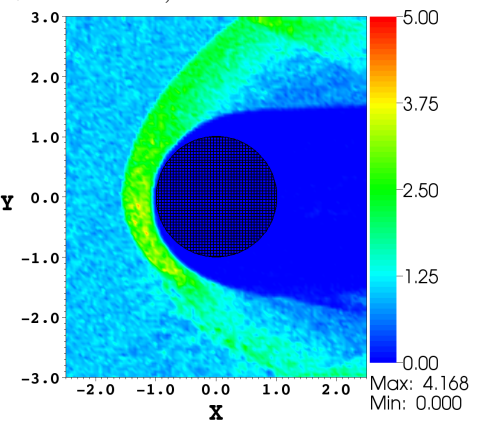

h) $n_{\mathrm{is}} / n_{\mathrm{sw}, 0}$

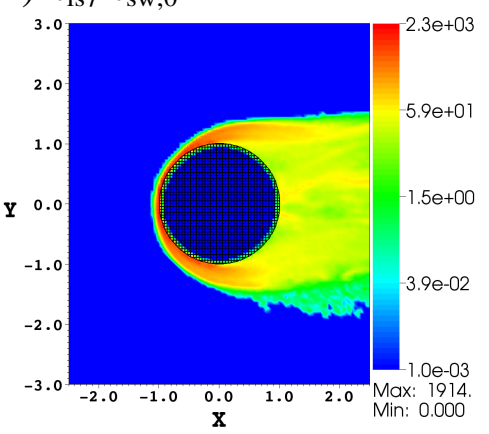

k) $\boldsymbol{v}_{\mathrm{is}} / v_{\mathrm{sw}, 0}$

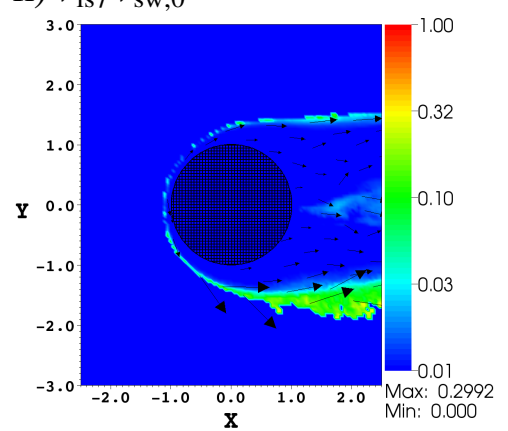

c) $\boldsymbol{B} / B_{\mathrm{sw}, 0}$

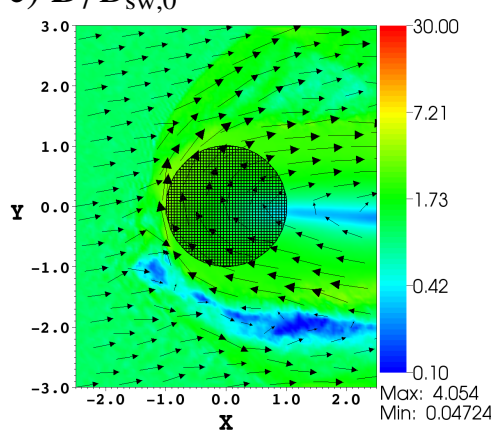

f) $n_{\mathrm{sw}} / n_{\mathrm{sw}, 0}$

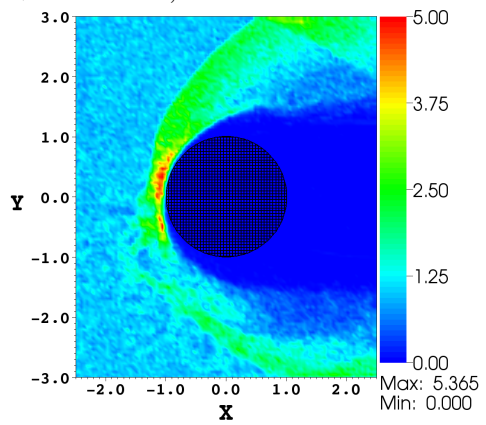

i) $n_{\mathrm{is}} / n_{\mathrm{sw}, 0}$

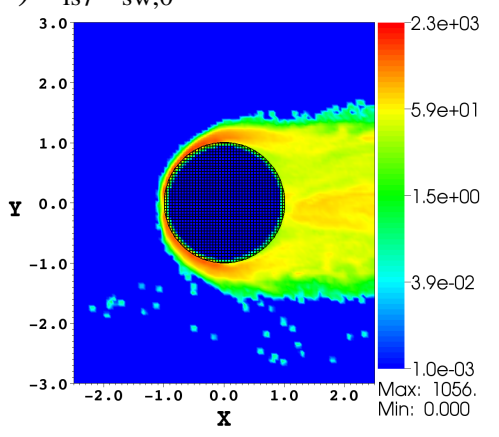

1) $v_{\text {is }} / v_{\mathrm{sw}, 0}$

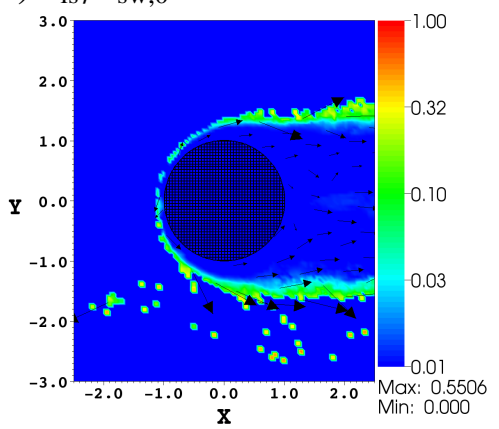

Figure 5.4: Simulation results in the form of equatorial plane cross sections for the perpendicular (left), intermediate (middle) and quasiparallel (right) simulation run. The first row (Figs. a-c) shows the magnetic field, the second row (Figs. d-f) the stellar wind density, the third row (Figs. g-i) the ionospheric density and the fourth row (Figs. j-1) the ionospheric velocity. 
a) $\boldsymbol{B} / B_{\mathrm{sw}, 0}$

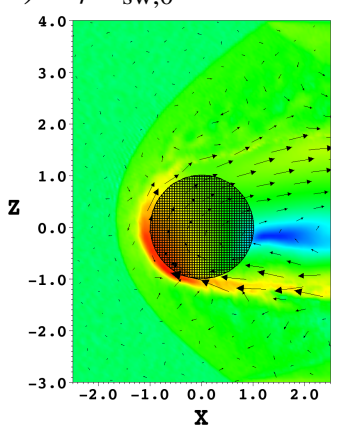

d) $n_{\mathrm{sw}} / n_{\mathrm{sw}, 0}$

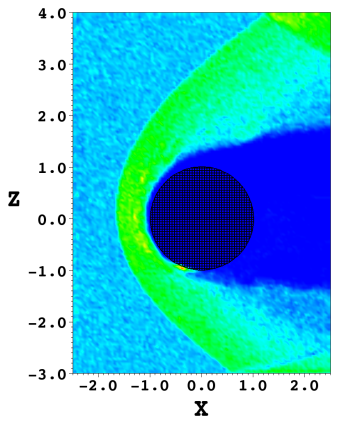

g) $n_{\text {is }} / n_{\mathrm{sw}, 0}$

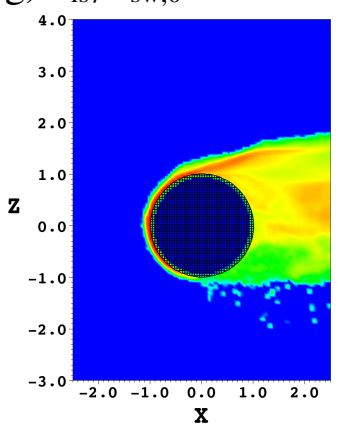

j) $\boldsymbol{v}_{\mathrm{is}} / \mathrm{v}_{\mathrm{sw}, 0}$

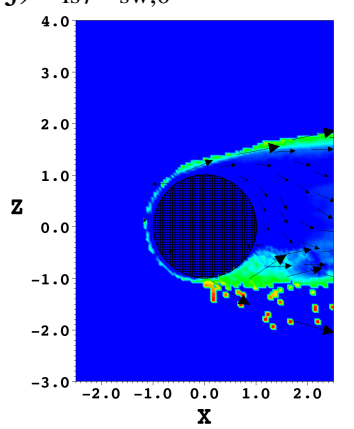

b) $\boldsymbol{B} / B_{\mathrm{sw}, 0}$
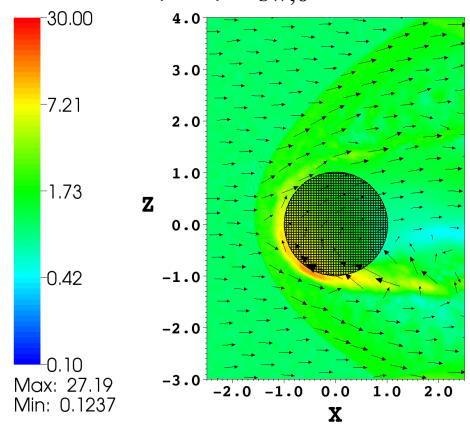

e) $n_{\mathrm{sw}} / n_{\mathrm{sw}, 0}$
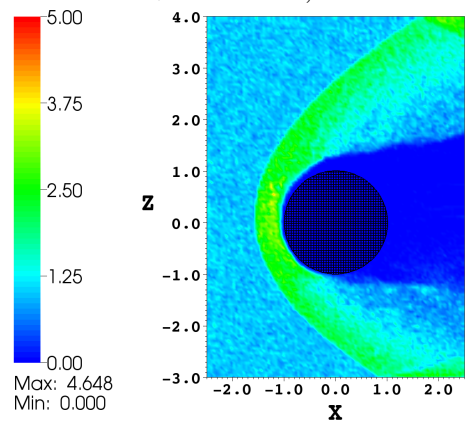

h) $n_{\text {is }} / n_{\mathrm{sw}, 0}$

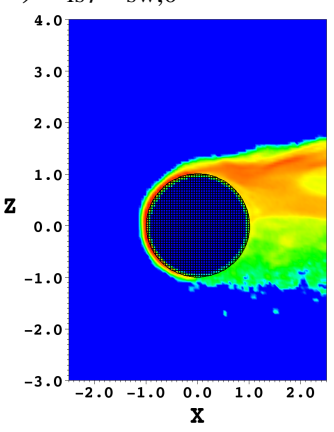

k) $\boldsymbol{v}_{\mathrm{is}} / v_{\mathrm{sw}, 0}$
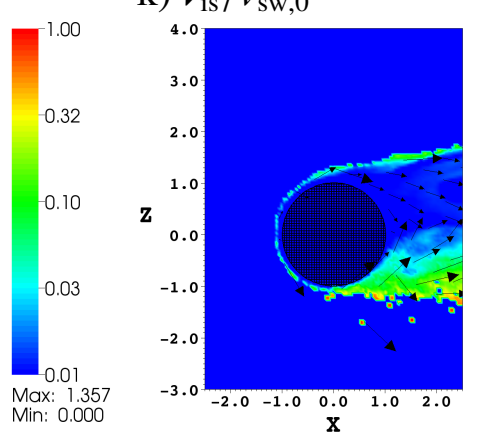

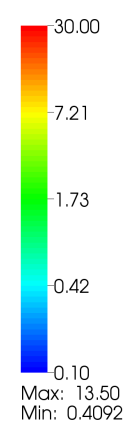

c) $\boldsymbol{B} / B_{\mathrm{sw}, 0}$

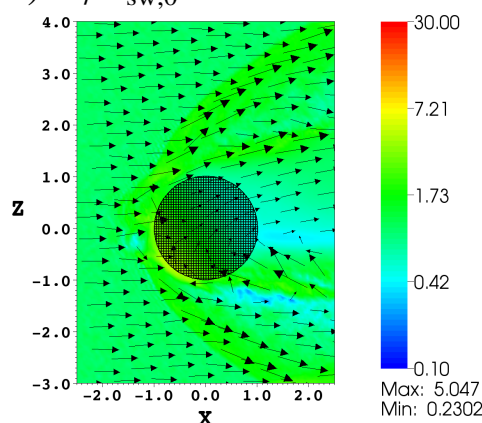

f) $n_{\mathrm{sw}} / n_{\mathrm{sw}, 0}$
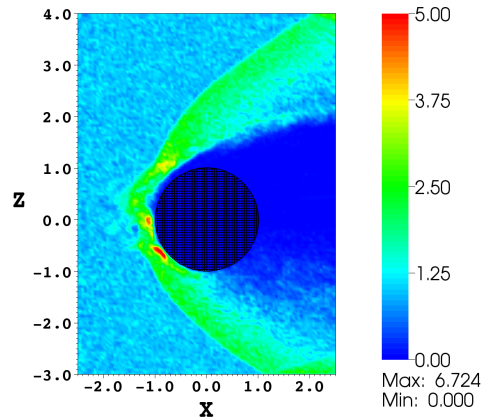

i) $n_{\text {is }} / n_{\mathrm{sw}, 0}$
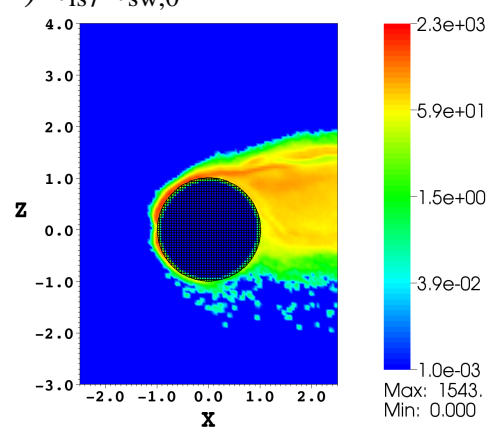

1) $\boldsymbol{v}_{\mathrm{is}} / v_{\mathrm{sw}, 0}$
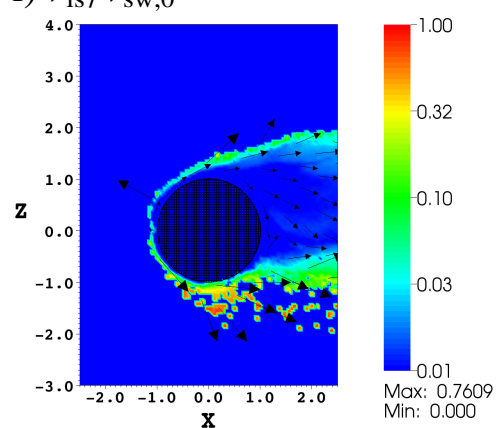

Figure 5.5: Simulation results in the form of polar plane cross sections for the perpendicular (left), intermediate (middle) and quasiparallel (right) simulation run. The first row (Figs. a-c) shows the magnetic field, the second row (Figs. d-f) the stellar wind density, the third row (Figs. g-i) the ionospheric density and the fourth row (Figs. j-1) the ionospheric velocity. 
a) $\boldsymbol{v}_{\mathrm{sw}} / v_{\mathrm{sw}, 0}$

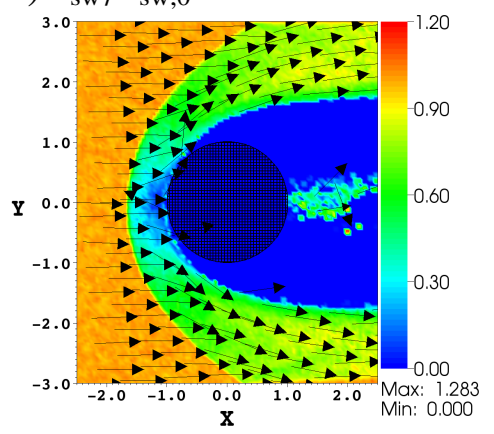

d) $\boldsymbol{E} /\left|v_{\mathrm{sw}, 0} B_{\mathrm{sw}, 0}\right|$

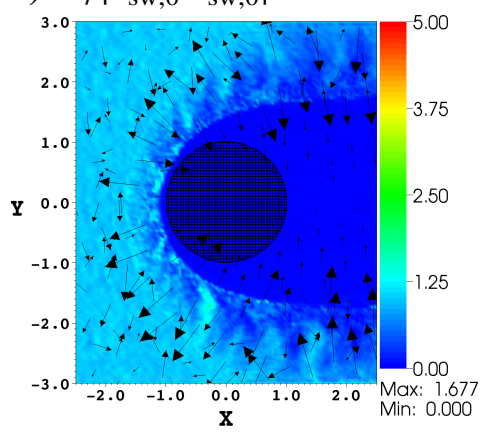

b) $\boldsymbol{v}_{\mathrm{sw}} / v_{\mathrm{sw}, 0}$

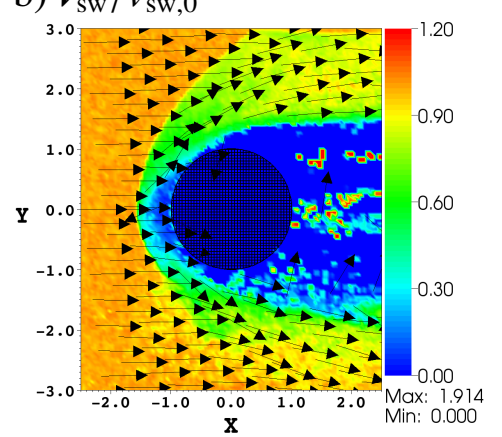

e) $\boldsymbol{E} /\left|v_{\mathrm{sw}, 0} B_{\mathrm{sw}, 0}\right|$

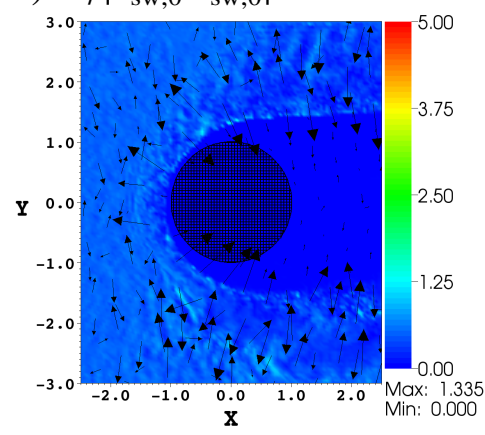

c) $\boldsymbol{v}_{\mathrm{sw}} / v_{\mathrm{sw}, 0}$

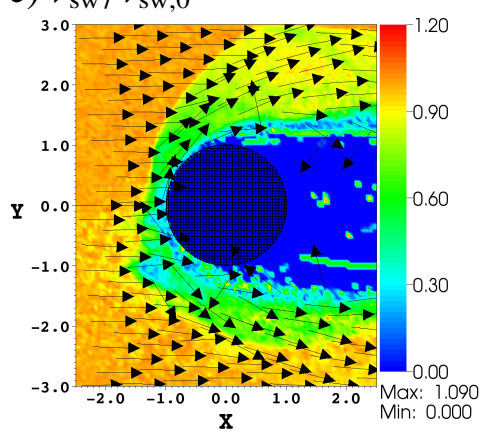

f) $\boldsymbol{E} /\left|v_{\mathrm{sw}, 0} \boldsymbol{B}_{\mathrm{sw}, 0}\right|$

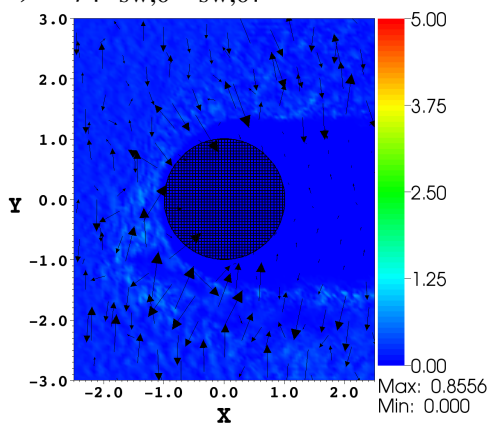

Figure 5.6: Simulation results in the form of equatorial plane cross sections for the perpendicular (left), intermediate (middle) and quasiparallel (right) simulation run. The first row (Figs. a-c) shows the stellar wind velocity and the second row (Figs. d-f) the electric field. 
a) $\boldsymbol{v}_{\mathrm{sw}} / v_{\mathrm{sw}, 0}$

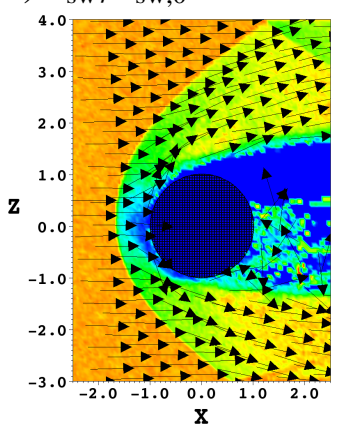

d) $\boldsymbol{E} /\left|v_{\mathrm{sw}, 0} B_{\mathrm{sw}, 0}\right|$

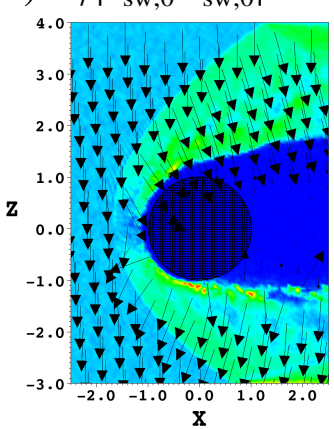

b) $\boldsymbol{v}_{\mathrm{sw}} / v_{\mathrm{sw}, 0}$
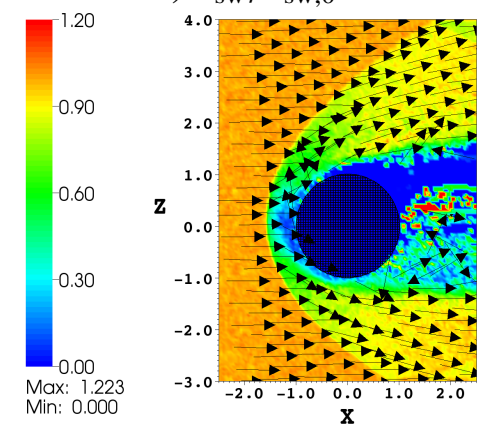

e) $\boldsymbol{E} /\left|v_{\mathrm{sw}, 0} B_{\mathrm{sw}, 0}\right|$
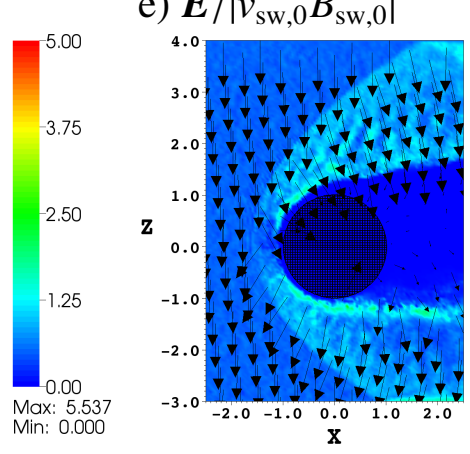

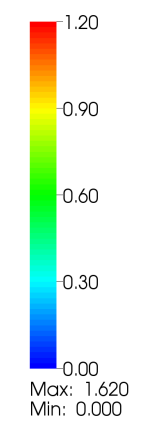

c) $\boldsymbol{v}_{\mathrm{sw}} / v_{\mathrm{sw}, 0}$

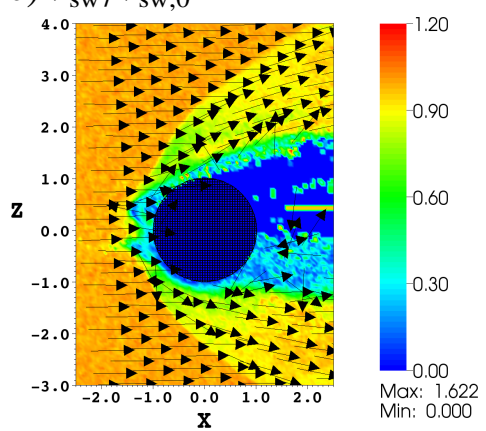

f) $\boldsymbol{E} /\left|v_{\mathrm{sw}, 0} B_{\mathrm{sw}, 0}\right|$

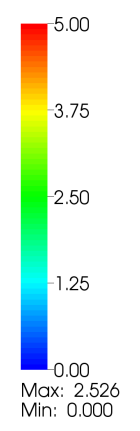

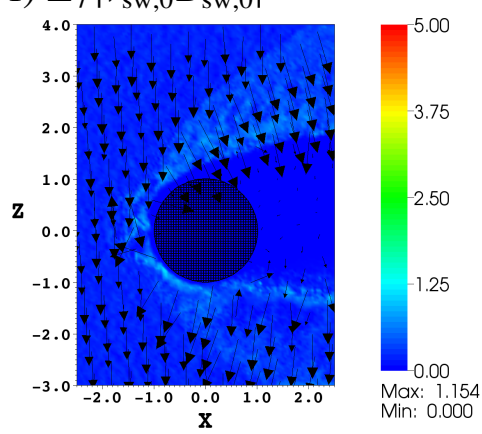

Figure 5.7: Simulation results in the form of polar plane cross sections for the perpendicular (left), intermediate (middle) and quasiparallel (right) simulation run. The first row (Figs. a-c) shows the stellar wind velocity and the second row (Figs. d-f) the electric field. 
a) $\boldsymbol{B} / B_{\mathrm{sw}, 0}$

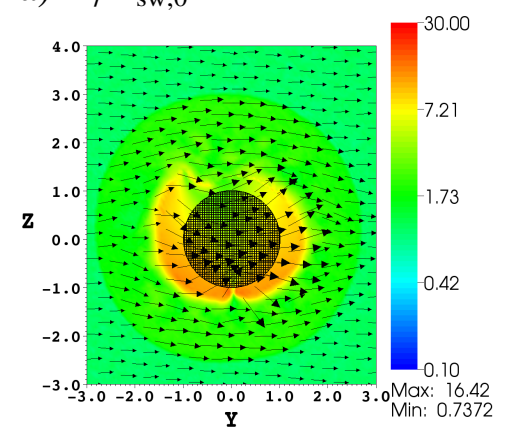

d) $n_{\text {is }} / n_{\text {sw }, 0}$

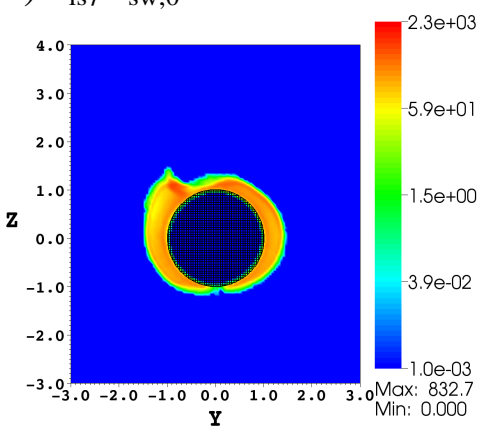

g) $v_{\text {is }} / v_{\mathrm{sw}, 0}$

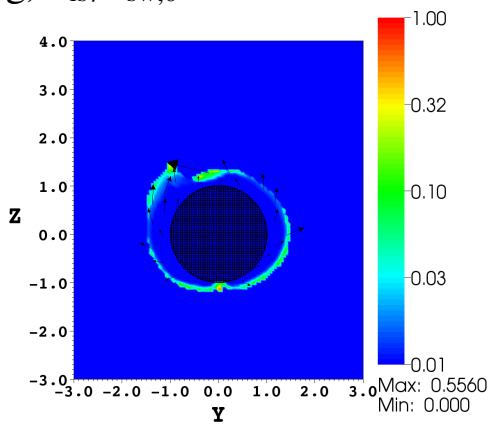

b) $\boldsymbol{B} / B_{\mathrm{sw}, 0}$

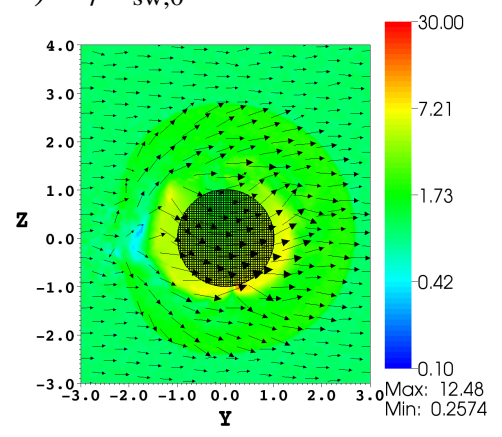

e) $n_{\text {is }} / n_{\mathrm{sw}, 0}$

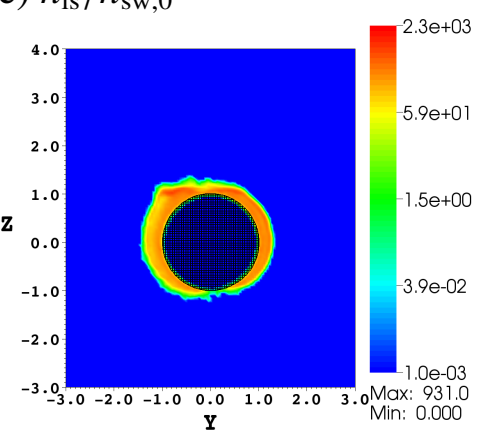

h) $\boldsymbol{v}_{\mathrm{is}} / v_{\mathrm{sw}, 0}$

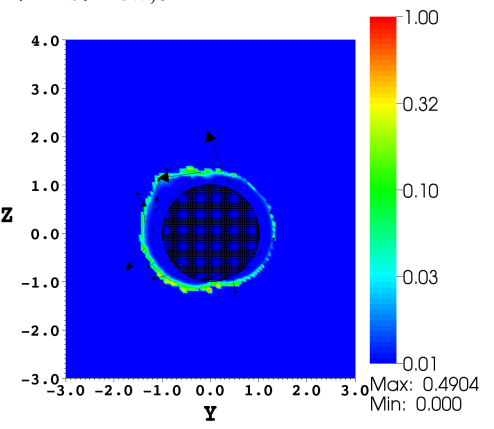

c) $\boldsymbol{B} / B_{\mathrm{sw}, 0}$

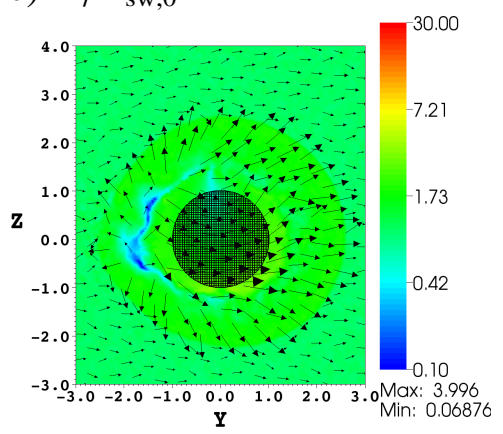

f) $n_{\mathrm{is}} / n_{\mathrm{sw}, 0}$

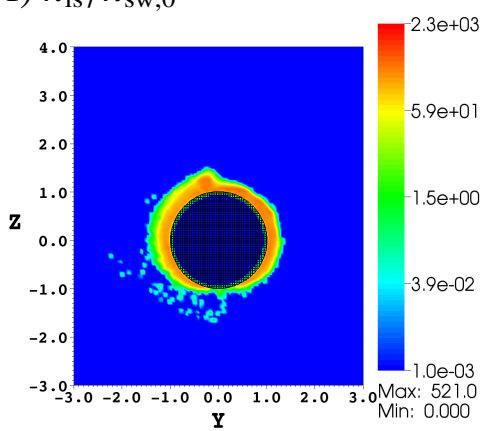

i) $\boldsymbol{v}_{\text {is }} / v_{\mathrm{sw}, 0}$

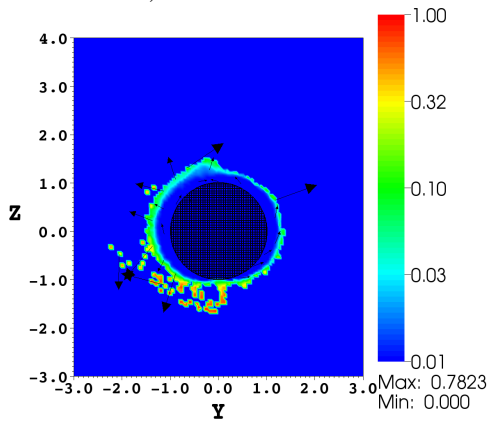

Figure 5.8: Simulation results in the form of terminator plane cross sections for the perpendicular (left), intermediate (middle) and quasiparallel (right) simulation run. The first row (Figs. a-c) shows the magnetic field, the second row (Figs. d-f) the ionospheric density and the third row (Figs. g-i) the ionospheric velocity. 
a) $\boldsymbol{v}_{E, z} / v_{\mathrm{sw}, 0}$

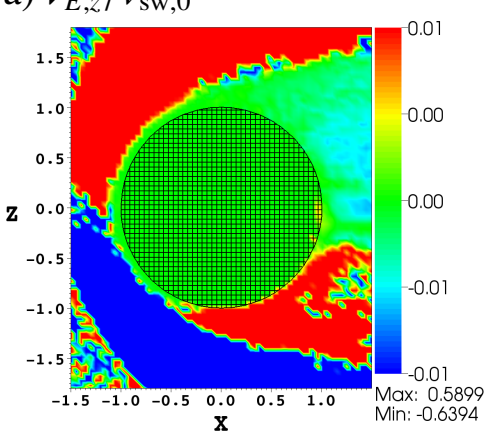

d) $\boldsymbol{v}_{E, z} / v_{\mathrm{sw}, 0}$

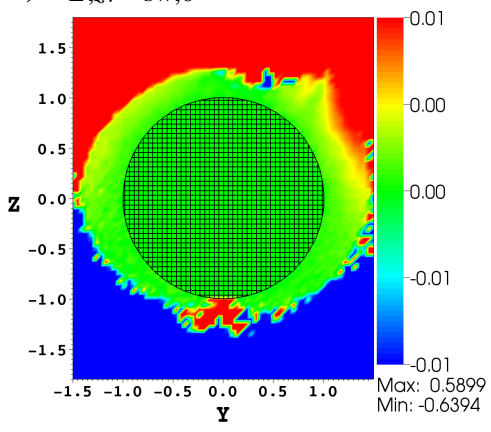

b) $\boldsymbol{v}_{E, z} / v_{\mathrm{sw}, 0}$

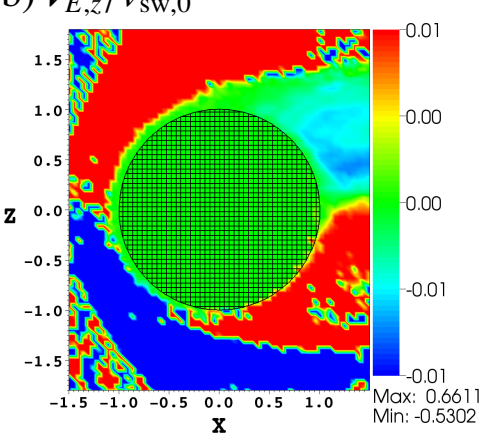

e) $\boldsymbol{v}_{E, z} / v_{\mathrm{sw}, 0}$

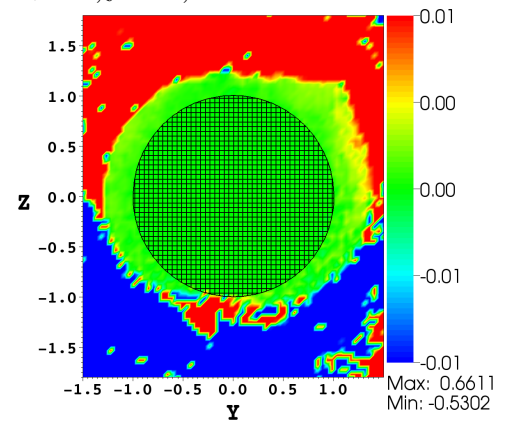

c) $v_{E, z} / v_{\mathrm{sw}, 0}$

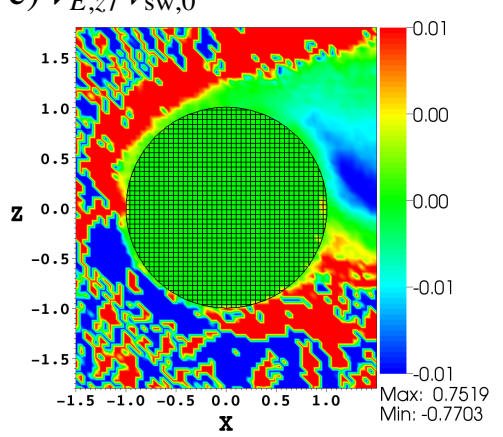

f) $\boldsymbol{v}_{E, z} / v_{\mathrm{sw}, 0}$

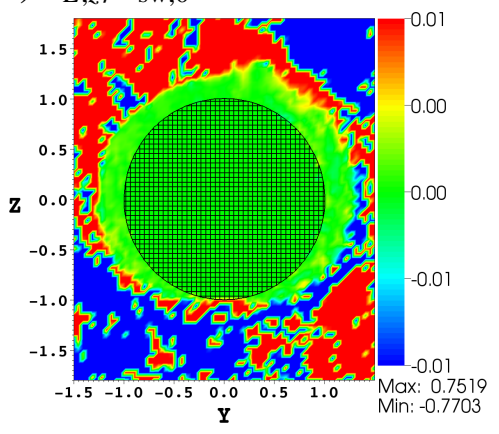

Figure 5.9: Simulation results in the form of polar cross sections (first row, Figs. a-c) and terminator cross sections (second row, Figs. d-f) for the perpendicular (left), intermediate (middle) and quasiparallel (right) simulation run. All figures show the $z$ component of the $\boldsymbol{E} \times \boldsymbol{B}$ drift velocity, representing the direction of magnetic drifts (see Eq. 5.12). It must of course be understood that these figures only show the value of the mathematical expression for $\boldsymbol{E} \times \boldsymbol{B}$ drift and not if drift motion actually occurs. The value is normalized using the undisturbed stellar wind velocity. The color scales are intentionally shrunk to less than the full range of values within the figures to better illustrate the area of interest. Therefore the colors are mostly saturated. Parts of the cross sections have been cut away for better overview. 


\section{Conclusions and outlook}

Only 15 years have passed since the first (confirmed) discovery of an extrasolar planet. More than 400 extrasolar planets have been discovered since and the number is still increasing rapidly. This has not only made research on planets outside the Solar System much less speculative, but has also opened up for the study of types of planets one never thought existed, close-in giant extrasolar planets being the primary example.

This work has aimed at being part of the effort to broaden the already well established study of solar wind interaction with various objects in the Solar System to stellar wind interaction with close-in extrasolar planets. These kinds of objects should experience several qualitatively different kinds of interaction which we do not see in the present-day Solar System.

The focus has been on hybrid simulation studies of two such types of stellar wind interaction: 1.) Interaction with a planet with an expanding atmosphere and therefore expanding ionosphere, and 2.) quasiparallel interaction. The hybrid simulation model itself is a model for simulating the time evolution of plasmas describing ions as particles and electrons as a fluid. Each study features three simulation runs, all identical with the exception of the parameter to be studied. The simulation studies have not tried to mimic any particular extrasolar planet but rather attempted to emulate generic but interesting stellar wind interaction scenarios for a close-in Earth-sized terrestrial planet without intrinsic magnetic field. Such planets have not been detected yet but are expected to be in the near future.

The purpose of the first simulation study was to study the stellar wind interaction with the expanding ionosphere of a planet with an extremely hydrodynamically expanding atmosphere. This is a type of atmospheres we do not have in the Solar System today. Results show that the expanding ionosphere both displaces all the major dayside structures upstream: bow shock, magnetic pile-up and ion composition boundary (ICB), as well as expands the entire interaction region. Interestingly enough, the dayside ionospheric expansion never manifests itself as a bulk flow. Instead most of the ionospheric bulk motion is immediately thermalized upon injection but does nonetheless still help push the ionosphere upstream by adding momentum to it. The expansion also changes the nature of the tail region, from the familiar structure of two lobes separated by a current sheet, to a wide region where the ionosphere can expand largely unaffected by the stellar wind. The great thickness of the ion producing region surrounding the planet resulting from the large extent of the neutral atmosphere, leads to more overlap with the dayside magnetosheath and stellar wind. This leads to more mixing of ionosphere and stellar wind and thus to a less well-defined ICB.

This first simulation study has in addition constituted the basis for a larger set of simulation runs, used to obtain standoff distances as a function of expansion velocity, stellar 
wind velocity and stellar wind density. These have then been used to test and improve an analytical estimate of the standoff distance for this type of systems. The current version of the analytical estimate, based on a pressure balance between the dynamic and thermal pressure for both stellar wind and expanding ionosphere, consistently underestimates the standoff distance from simulation runs, at least in part because it neither takes into account the difference in pressure between the upstream stellar wind and ionopause pressure, nor the thermalization of dayside ionospheric bulk flow. One can however attain a fairly good fit if one assumes a higher effective ionospheric production rate.

The second simulation study investigated the consequences of quasiparallel stellar wind interaction with a close-in Venus-like planet, i.e. what happens when the perpendicular component of the IMF is small which may happen for certain close-in orbits. Many of the generic features of stellar wind interaction depend on the existence of a non-zero perpendicular IMF component which is why one can expect this to produce a very different type of interaction. The study shows how many of these features weaken when the perpendicular component of the IMF decreases. A large part of the dayside shock surface is replaced by a vaguely defined parallel shock which destroys the strict division between magnetosheath and upstream stellar wind and changes the magnetosheath itself. The draping of magnetic field lines leads to the creation of something that resembles a second current sheet next to the planet. Furthermore, the stellar wind penetrates farther into the ionosphere for lower IMF angles. In addition, the simulation runs all have the odd feature that the ionosphere is strangely compressed on one side, and for which no adequate explanation has been found. It can not be excluded that it is due to numerical error.

While the simulation studies in this work are not directly connected to observations, they should be useful for the understanding of some qualitatively different types of stellar wind interactions of which we have only limited experience from the Solar System. This kind of understanding should be relevant for in particular the evolution of the atmospheres of these planets.

Although hybrid simulation model has its advantages in the form of being able describe arbitrary ion velocity distributions and kinetic effects, it is not perfect for close-in planets due to the stronger magnetic fields. The overall magnetic field strength sets a numerical limit since it increases the ion gyration frequencies, which in turn requires smaller time steps in order to resolve all the the gyrations. Therefore one is effectively forced to work in a low magnetic field limit. Close-in planets are in principle in the domain of MHD. Another difficulty throughout this work is the need to find a both reasonable and interesting set of parameters to describe one's planet. Since the data on the great majority of extrasolar planets is very scarce, one is forced to make a lot of assumptions and educated guesses.

Nonetheless, there is more one can do continuing on the simulation studies featured here. The simulation study of expanding atmospheres and ionospheres uses an in all likelihood quite extreme range of expansion velocities. Nonetheless, these kinds of hydrodynamically expanding atmospheres are interesting not only because of their expansion but also because of their extent, up to altitudes of several planetary radii. Therefore it could be interesting to study this again, but focusing on having a more physically correct, atmospheric and ionospheric model, possibly combined with a quasiparallel interaction.

The study of quasiparallel interactions is in principle not finished since it does not 
include a purely parallel simulation run. It would in particular be interesting to try to confirm if the ionosphere really is less able to resist the stellar wind in a parallel interaction and try to quantify it. This could have consequences for the chemistry of the upper atmosphere and for atmospheric escape. It is also not obvious that the IMF angle is the right parameter to vary in parameter studies like ours.

The great diversity of extrasolar planets and their host stars does of course open up for varying yet other parameters. For example, the stellar wind parameters vary with both age and type of star. In the end, extrasolar planets are still to some extent a moving, or growing, target since the known population is still growing, new types of planets are still being found and astronomers are still learning how to extract more and more data from these systems. New and proposed space missions such as Kepler, the Terrestrial Planet Finder and Darwin promise to be able to even detect Earth-like planets. 



\section{A Standoff distances from estimates and simulations}

\begin{tabular}{c|ccc}
$u\left[\mathrm{~km} \mathrm{~s}^{-1}\right]$ & $R_{\mathrm{s}, \text { sim. }}\left[R_{\mathrm{p}}\right]$ & $R_{\mathrm{s}, 1}\left[R_{\mathrm{p}}\right]$ & $R_{\mathrm{s}, 2}\left[R_{\mathrm{p}}\right]$ \\
\hline 0 & 1.8309 & - & - \\
50 & 2.1741 & 1.6202 & 1.9843 \\
75 & 2.3788 & 1.9340 & 2.3686 \\
100 & 2.7690 & 2.2125 & 2.7097 \\
125 & 3.0176 & 2.4628 & 3.0163
\end{tabular}

Table A.1: Standoff distances calculated from simulations and analytical estimates for a series of simulations runs where we have varied the initial ionospheric radial velocity $u$. The data in this table are plotted and explained in Fig. 4.1.

\begin{tabular}{c|ccc}
$n_{\mathrm{sw}}\left[\mathrm{cm}^{-3}\right]$ & $R_{\mathrm{s}, \mathrm{sim} .}\left[R_{\mathrm{p}}\right]$ & $R_{\mathrm{s}, 1}\left[R_{\mathrm{p}}\right]$ & $R_{\mathrm{s}, 2}\left[R_{\mathrm{p}}\right]$ \\
\hline 600 & 2.4574 & 2.1433 & 2.6249 \\
750 & 2.3211 & 1.9170 & 2.3478 \\
900 & 2.2213 & 1.7500 & 2.1433 \\
1050 & 2.1741 & 1.6202 & 1.9843 \\
1200 & 2.0505 & 1.5155 & 1.8561 \\
1350 & 2.0661 & 1.4288 & 1.7500
\end{tabular}

Table A.2: Standoff distances calculated from simulations and analytical estimates for a series of simulations runs where we have varied the stellar wind density $n_{\mathrm{sw}}$. The data in this table are plotted and explained in Fig. 4.2 a.

\begin{tabular}{c|lll}
$v_{\mathrm{sw}}\left[\mathrm{km} \mathrm{s}^{-1}\right]$ & $R_{\mathrm{s}, \text { sim. }}\left[R_{\mathrm{p}}\right]$ & $R_{\mathrm{s}, 1}\left[R_{\mathrm{p}}\right]$ & $R_{\mathrm{s}, 2}\left[R_{\mathrm{p}}\right]$ \\
\hline 200 & 2.8240 & 2.3380 & 2.8635 \\
250 & 2.3761 & 1.9172 & 2.3481 \\
300 & 2.1741 & 1.6202 & 1.9843 \\
400 & 1.9918 & 1.2326 & 1.5096 \\
500 & 1.8549 & 0.9928 & 1.2159
\end{tabular}

Table A.3: Standoff distances calculated from simulations and analytical estimates for a series of simulations runs where we have varied the stellar wind velocity $v_{\mathrm{sw}}$. The data in this table are plotted and explained in Fig. 4.2p. 



\section{B Magnetic drifts}

To investigate magnetic drifts as the possible explanation for the depressed ionosphere on the negative $z$ side of the planet surface as visible in Figs. 5.5g-i and 5.8d-f, we must first ensure that the typical size of gyrations, $r_{\mathrm{g}, \mathrm{O}^{+}}$, for oxygen ions well inside the oxygen dominated ionosphere is much smaller than the size of the region. Using the initial thermal velocity $v_{\text {th }} \sim 2.2 \mathrm{~km} \mathrm{~s}^{-1}$ of the oxygen ions and the local magnetic field strength $B \sim 20 B_{\mathrm{sw}, 0}$ we obtain

$$
r_{\mathrm{g}, \mathrm{O}^{+}} \sim 10^{-4} R_{\mathrm{p}}
$$

which we will regard as small enough.

To calculate the total drift velocity we will need the gradient drift velocity $\boldsymbol{v}_{\nabla}$

$$
\boldsymbol{v}_{\nabla}=\frac{m \boldsymbol{v}_{\perp}^{2}}{2 q} \frac{\boldsymbol{B} \times \nabla B}{B^{3}}
$$

and the curvature drift velocity $\boldsymbol{v}_{R}$

$$
\boldsymbol{v}_{R}=\frac{m \boldsymbol{v}_{\|}^{2}}{q} \frac{\boldsymbol{R}_{\boldsymbol{c}} \times \boldsymbol{B}}{R_{c}^{2} B^{2}}
$$

where $m$ is the particle mass, $\boldsymbol{v}_{\perp}$ and $\boldsymbol{v}_{\|}$are the particle velocity perpendicular and parallel to the magnetic field, $q$ is the particle charge and $\boldsymbol{R}_{\boldsymbol{c}}$ is the vector defining the local radius of curvature for the field line. $\boldsymbol{R}_{\boldsymbol{c}}$ can be expressed as a local quantity with the expression

$$
\boldsymbol{R}_{c}=-\frac{\hat{B} \cdot \nabla \hat{B}}{|\hat{B} \cdot \nabla \hat{B}|^{2}}
$$

where $\hat{B}=\boldsymbol{B} / B$. Judging from the geometry of draping we can already now conclude that the curvature drift should have a negative $z$ component, i.e. an effect opposite to what we are looking for.

If we assume that the oxygen ions have an isotropic distribution of velocities then we obtain

$$
m v_{\perp}^{2}=2 m v_{\|}^{2}
$$

and

$$
\frac{1}{2} m\left(\boldsymbol{v}_{\perp}^{2}+\boldsymbol{v}_{\|}^{2}\right)=\frac{3}{2} k_{\mathrm{B}} T_{\mathrm{i}}
$$

where $T_{\mathrm{i}}$ is the ion temperature. If we also eliminate $\boldsymbol{R}_{\boldsymbol{c}}$ we can finally add the two drift velocities together to a drift velocity averaged over many ions.

$$
\begin{aligned}
\boldsymbol{v}_{\nabla}+\boldsymbol{v}_{R} & =\frac{m \boldsymbol{v}_{\perp}^{2}}{2 q} \frac{\boldsymbol{B} \times \nabla B}{B^{3}}-\frac{m \boldsymbol{v}_{\|}^{2}}{q} \frac{(\boldsymbol{B} \cdot \nabla \hat{B}) \times \boldsymbol{B}}{B^{3}} \\
& =\frac{k_{\mathrm{B}} T_{\mathrm{i}}}{2 q} \cdot \frac{\boldsymbol{B} \times \nabla B-(\boldsymbol{B} \cdot \nabla \hat{B}) \times \boldsymbol{B}}{B^{3}}
\end{aligned}
$$


a) $2 q /\left(k_{\mathrm{B}} T_{\mathrm{i}}\right)\left(v_{\nabla}+v_{R}\right)_{z}$

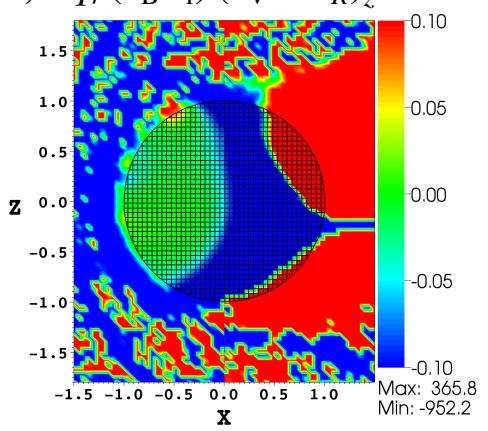

d) $2 q /\left(k_{\mathrm{B}} T_{\mathrm{i}}\right)\left(v_{\nabla}+v_{R}\right)_{z}$

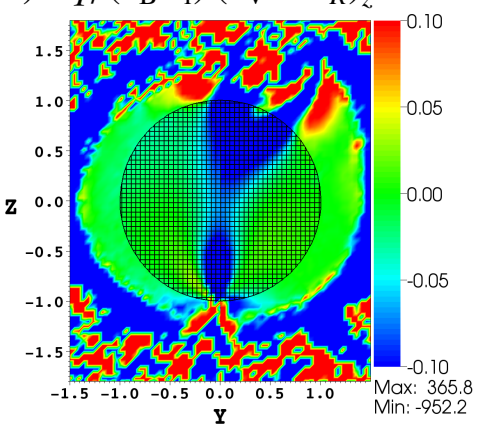

b) $2 q /\left(k_{\mathrm{B}} T_{\mathrm{i}}\right)\left(v_{\nabla}+v_{R}\right)_{z}$

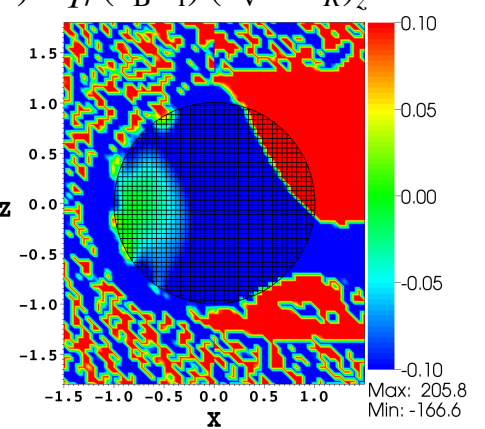

e) $2 q /\left(k_{\mathrm{B}} T_{\mathrm{i}}\right)\left(\boldsymbol{v}_{\nabla}+\boldsymbol{v}_{R}\right)_{z}$

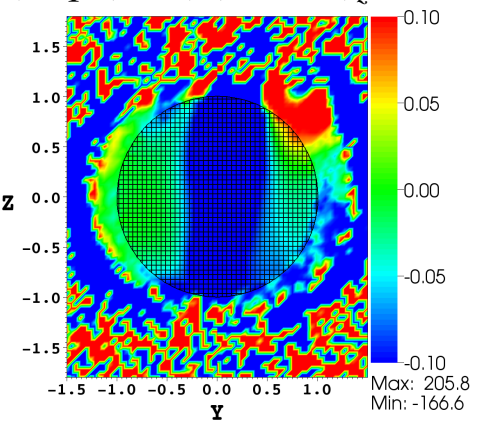

c) $2 q /\left(k_{\mathrm{B}} T_{\mathrm{i}}\right)\left(v_{\nabla}+v_{R}\right)_{z}$

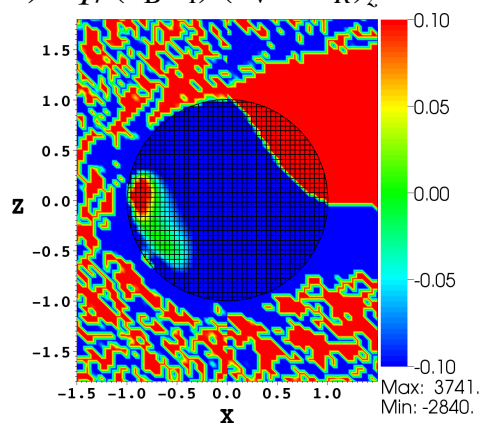

f) $2 q /\left(k_{\mathrm{B}} T_{\mathrm{i}}\right)\left(\boldsymbol{v}_{\nabla}+\boldsymbol{v}_{R}\right)_{z}$

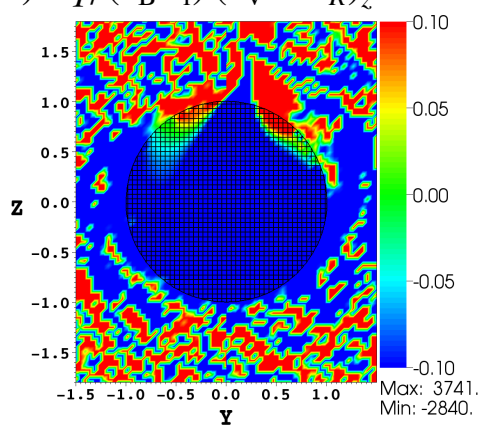

Figure B.1: Simulation results in the form of polar cross sections (first row, Figs. a-c) and terminator cross sections (second row, Figs. d-f) for the perpendicular (left), intermediate (middle) and quasiparallel (right) simulation run. All figures show the $z$ component of the vector quantity $2 q /\left(k_{\mathrm{B}} T_{\mathrm{i}}\right)\left(\boldsymbol{v}_{\nabla}+v_{R}\right)_{z}$, representing the $z$ direction of magnetic drifts (see Eq. B.8). It must of course be understood that these figures only show the value of the mathematical expression for magnetic drift and not if drift motion actually occurs. The value is expressed in units of $B_{\mathrm{sw}, 0}{ }^{-1} R_{\mathrm{p}}{ }^{-1}$. The color scales are intentionally shrunk to less than the full range of values within the figures to better illustrate the area of interest. Therefore the colors are mostly saturated. Parts of the cross sections have been cut away for better overview.

This way we can plot the direction of $\boldsymbol{v}_{\nabla}+\boldsymbol{v}_{R}$ and see if there is any tendency of drift toward the planet. The result can be found in Figs. B.1. Note that the figures have their color scales intentionally saturated to make it easier to see the sign of the drift in the $z$ direction. Using the ionospheric temperature of $T_{\mathrm{i}}=3000 \mathrm{~K}$ implies that the value 0.1 from Figs. B.1 is equivalent to a drift velocity of $0.17 \mathrm{~m} \mathrm{~s}^{-1}$.

Comparing with the depressed ionosphere in Figs. 5.5g-i and 5.8 d-f one can see a certain agreement between the depressed ionosphere on the negative $z$ side with drift in the positive $z$ direction for the perpendicular simulation run while the correlation is weak and non-existent for the intermediate and quasiparallel run respectively. 


\section{Bibliography}

Bagdonat, T., 2005, Hybrid simulation of weak comets, Ph.D. thesis, Technische Universität Braunschweig

Bagdonat, T., Motschmann, U., 2002, Journal of Computational Physics, 183, 470-485

Bastian, T. S., Dulk, G. A., Leblanc, Y., 2000, A Search for Radio Emission from Extrasolar Planets, Astrophysical Journal, 545, 1058-1063

Baumjohann, W., Treumann, R. A., 1996, Basic space plasma physics, London: Imperial College Press

Boesswetter, A., Bagdonat, T., Motschmann, U., Sauer, K., 2004, Plasma boundaries at Mars: a 3-D simulation study, Annales Geophysicae, 22, 4363-4379

Boesswetter, A., Simon, S., Bagdonat, T., Motschmann, U., Fränz, M., Roussos, E., Krupp, N., Woch, J., Schuele, J., Barabash, S., Lundin, R., 2007, Comparison of plasma data from ASPERA-3/Mars-Express with a 3-D hybrid simulation, Annales Geophysicae, $25,1851-1864$

Borovsky, J. E., Funsten, H. O., 2003, Role of solar wind turbulence in the coupling of the solar wind to the Earth's magnetosphere, Journal of Geophysical Research (Space Physics), 108, 1246

Burgess, D., 1989, Cyclic behavior at quasi-parallel collisionless shocks, Geophysics Research Letters, 16, 345-348

Campbell, B., Walker, G. A. H., Yang, S., 1988, A search for substellar companions to solar-type stars, Astrophysical Journal, 331, 902-921

Chamberlain, J. W., Hunten, D. M., 1987, Theory of planetary atmospheres. An introduction to their physics and chemistry.

Charbonneau, D., Brown, T. M., Burrows, A., Laughlin, G., 2007, When Extrasolar Planets Transit Their Parent Stars, in Protostars and Planets V, (Eds.) B. Reipurth, D. Jewitt, K. Keil, pp. 701-716

Dobrovolskis, A. R., Borucki, W. J., 1996, Influence of Jovian Extrasolar Planets on Transits of Inner Planets, in Bulletin of the American Astronomical Society, vol. 28 of Bulletin of the American Astronomical Society, pp. 1112-1112 
Farrell, W. M., Desch, M. D., Zarka, P., 1999, On the possibility of coherent cyclotron emission from extrasolar planets, Journal of Geophysics Research, 104, 14 025-14 032

Farrell, W. M., Desch, M. D., Lazio, T. J., Bastian, T., Zarka, P., 2003, Limits on the Magnetosphere/Stellar Wind Interactions for the Extrasolar Planet about Tau Bootis, in Scientific Frontiers in Research on Extrasolar Planets, (Eds.) D. Deming, S. Seager, vol. 294 of Astronomical Society of the Pacific Conference Series, pp. 151-156

Filippychev, D. S., 2000, Hybrid simulation of space plasmas: Models with massless fluid representation of electrons. I. Collisionless shocks, Computational Mathematics and Modeling, 11, 15-39

Grießmeier, J., Stadelmann, A., Grenfell, J. L., Lammer, H., Motschmann, U., 2009, On the protection of extrasolar Earth-like planets around K/M stars against galactic cosmic rays, Icarus, 199, 526-535, arXiv:0902.0952

Griessmeier, J.-M., Stadelmann, A., Penz, T., Lammer, H., Selsis, F., Ribas, I., Guinan, E. F., Motschmann, U., Biernat, H. K., Weiss, W. W., 2004, The effect of tidal locking on the magnetospheric and atmospheric evolution of "Hot Jupiters", Astronomy and Astrophysics, 425, 753-762

Griessmeier, J.-M., Preusse, S., Khodachenko, M., Motschmann, U., Mann, G., Rucker, H. O., 2007a, Exoplanetary radio emission under different stellar wind conditions, Planetary Space Science, 55, 618-630

Griessmeier, J.-M., Zarka, P., Spreeuw, H., 2007b, Predicting low-frequency radio fluxes of known extrasolar planets, Astronomy and Astrophysics, 475, 359-368, arXiv: 0806.0327

Griessmeier, J.-M., Johansson, E. P. G., Mueller, J., Bagdonat, T., Motschmann, U., 2010, The magnetopause location in the case of an expanding ionosphere, In preparation

Hatzes, A. P., Cochran, W. D., Endl, M., McArthur, B., Paulson, D. B., Walker, G. A. H., Campbell, B., Yang, S., 2003, A Planetary Companion to $\gamma$ Cephei A, Astrophysical Journal, 599, 1383-1394, arXiv: astro-ph/0305110

Henry, G. W., Baliunas, S. L., Donahue, R. A., Soon, W. H., Saar, S. H., 1997, Properties of Sun-like Stars with Planets: 51 Pegasi, 47 Ursae Majoris, 70 Virginis, and HD 114762, Astrophysical Journal, 474, 503-510

Holman, M. J., Murray, N. W., 2005, The Use of Transit Timing to Detect TerrestrialMass Extrasolar Planets, Science, 307, 1288-1291

Holmström, M., Ekenbäck, A., Selsis, F., Penz, T., Lammer, H., Wurz, P., 2008, Energetic neutral atoms as the explanation for the high-velocity hydrogen around HD 209458b, Nature, 451, 970-972, arXiv:0802 .2764

Huebner, W. F., Keady, J. J., Lyon, S. P., 1992, Solar photo rates for planetary atmospheres and atmospheric pollutants, Astrophysics and Space Science, 195, 1-289 
Ip, W.-H., Kopp, A., Hu, J.-H., 2004, On the Star-Magnetosphere Interaction of Close-in Exoplanets, Astrophysical Journal, Letters, 602, L53-L56

Johansson, E. P. G., Bagdonat, T., Motschmann, U., 2009, Consequences of expanding exoplanetary atmospheres for magnetospheres, Astronomy and Astrophysics, 496, 869-877

Johansson, E. P. G., Mueller, J., Motschmann, U., 2010, Hybrid simulations of quasiparallel stellar wind interaction with close-in exoplanets, In preparation

Kallio, E., Janhunen, P., 2003, Modelling the solar wind interaction with Mercury by a quasi-neutral hybrid model, Annales Geophysicae, 21, 2133-2145

Kasting, J. F., Pollack, J. B., 1983, Loss of water from Venus. I - Hydrodynamic escape of hydrogen, Icarus, 53, 479-508

Khodachenko, M. L., Lammer, H., Lichtenegger, H. I. M., Langmayr, D., Erkaev, N. V., Grießmeier, J., Leitner, M., Penz, T., Biernat, H. K., Motschmann, U., Rucker, H. O., 2007, Mass loss of Hot Jupiters - Implications for CoRoT discoveries. Part I: The importance of magnetospheric protection of a planet against ion loss caused by coronal mass ejections, Planetary Space Science, 55, 631-642

Kivelson, M. G., Russell, C. T., 1995, Introduction to Space Physics, Cambridge University Press

Kriegel, H., Simon, S., Müller, J., Motschmann, U., Saur, J., Glassmeier, K., Dougherty, M. K., 2009, The plasma interaction of Enceladus: 3D hybrid simulations and comparison with Cassini MAG data, Planetary Space Science, 57, 2113-2122

Kulikov, Y. N., Lammer, H., Lichtenegger, H. I. M., Penz, T., Breuer, D., Spohn, T., Lundin, R., Biernat, H. K., 2007, A Comparative Study of the Influence of the Active Young Sun on the Early Atmospheres of Earth, Venus, and Mars, Space Science Reviews, 129, 207-243

Lammer, H., Kasting, J. F., Chassefière, E., Johnson, R. E., Kulikov, Y. N., Tian, F., 2008, Atmospheric Escape and Evolution of Terrestrial Planets and Satellites, Space Science Reviews, 139, 399-436

Lanza, A. F., 2009, Stellar coronal magnetic fields and star-planet interaction, Astronomy and Astrophysics, 505, 339-350, arXiv:0906. 1738

Latham, D. W., Stefanik, R. P., Mazeh, T., Mayor, M., Burki, G., 1989, The unseen companion of HD114762 - A probable brown dwarf, Nature, 339, 38-40

Lazio, T. J. W., Farrell, W. M., Dietrick, J., Greenlees, E., Hogan, E., Jones, C., Hennig, L. A., 2004, The Radiometric Bode's Law and Extrasolar Planets, Astrophysical Journal, 612, 511-518

Lecavelier des Etangs, A., Vidal-Madjar, A., McConnell, J. C., Hébrard, G., 2004, Atmospheric escape from hot Jupiters, Astronomy and Astrophysics, 418, L1-L4, arXiv:astro-ph/0403369 
Lin, D. N. C., 2006, Overview and prospective in theory and observation of planet formation, pp. 256-262, Cambridge University Press

Lovis, C., Mayor, M., Udry, S., 2006, From hot Jupiters to hot Neptunes.. and below, pp. 203-215, Cambridge University Press

Mann, G., Jansen, F., MacDowall, R. J., Kaiser, M. L., Stone, R. G., 1999, A heliospheric density model and type III radio bursts, Astronomy and Astrophysics, 348, 614-620

Mariani, F., Neubauer, F. M., 1990, The Interplanetary Magnetic Field, pp. 183-206

Martinecz, C., Boesswetter, A., Fraenz, M., Roussos, E., Woch, J., Krupp, N., Dubinin, E., Motschmann, U., Wiehle, S., Simon, S., Barabash, S., Lundin, R., Zhang, T. L., Lammer, H., Lichtenegger, H., Kulikov, Y., 2009, Plasma environment of Venus: Comparison of Venus Express ASPERA-4 measurements with 3-D hybrid simulations, Journal of Geophysical Research (Space Physics), 114, E00B30

Mayor, M., Queloz, D., 1995, A Jupiter-mass companion to a solar-type star, Nature, 378, 355-359

Mueller, J., Pringle, G., Schuele, J., Motschmann, U., 2010, In preparation

Parker, E. N., 1958, Dynamics of the Interplanetary Gas and Magnetic Fields., Astrophysical Journal, 128, 664-676

Perryman, M. A. C., 2000, Extra-solar planets, Reports on Progress in Physics, 63, 12091272, arXiv:astro-ph/0005602

Preusse, S., Kopp, A., Büchner, J., Motschmann, U., 2005, Stellar wind regimes of closein extrasolar planets, Astronomy and Astrophysics, 434, 1191-1200

Preusse, S., Kopp, A., Büchner, J., Motschmann, U., 2007, MHD simulation scenarios of the stellar wind interaction with Hot Jupiter magnetospheres, Planetary Space Science, $55,589-597$

Raymond, S. N., Mandell, A. M., Sigurdsson, S., 2006, Exotic Earths: Forming Habitable Worlds with Giant Planet Migration, Science, 313, 1413-1416, arXiv:astro-ph/ 0609253

Ribas, I., Guinan, E. F., Güdel, M., Audard, M., 2005, Evolution of the Solar Activity over Time and Effects on Planetary Atmospheres. I. High-Energy Irradiances (1-1700 Å), Astrophysical Journal, 622, 680-694, arXiv: astro-ph/0412253

Richards, P. G., Fennelly, J. A., Torr, D. G., 1994, EUVAC: A solar EUV flux model for aeronomic calculations, Journal of Geophysics Research, 99, 8981-8992

Roussos, E., Mueller, J., Simon, S., Boesswetter, A., Motschmann, U., Krupp, N., Fraenz, M., Woch, J., Khurana, K. K., Dougherty, M. K., 2008, Plasma and fields in the wake of rhea: 3-d hybrid simulation and comparison with cassini data, Annales Geophysicae, 26, 619-637, ISSN 0992-7689 
Sartoretti, P., Schneider, J., 1999, On the detection of satellites of extrasolar planets with the method of transits, Astronomy and Astrophysics, Supplement, 134, 553-560

Schunk, R. W., Nagy, A. F., 2000, Ionospheres: Physics, Plasma Physics, and Chemistry, Cambridge University Press

Schwenn, R., Marsch, E., 1990, Physics of the Inner Heliosphere I. Large-Scale Phenomena.

Schwenn, R., Marsch, E., 1991, Physics of the Inner Heliosphere II. Particles, Waves and Turbulence., Springer-Verlag Berlin Heidelberg New York

Seager, S., Liang, M.-C., Parkinson, C. D., Yung, Y. L., 2005, Exoplanet Atmospheres and Photochemistry, in Astrochemistry: Recent Successes and Current Challenges, (Eds.) D. C. Lis, G. A. Blake, E. Herbst, vol. 231 of IAU Symposium, pp. 491-498

Shkolnik, E., Aigrain, S., Cranmer, S., Fares, R., Fridlund, M., Pont, F., Schmitt, J., Smith, A., Suzuki, T., 2009, Star-Planet Interactions, in American Institute of Physics Conference Series, (Ed.) E. Stempels, vol. 1094 of American Institute of Physics Conference Series, pp. 275-282

Simon, S., Boesswetter, A., Bagdonat, T., Motschmann, U., Glassmeier, K.-H., 2006, Plasma environment of Titan: a 3-D hybrid simulation study, Annales Geophysicae, 24, 1113-1135

Simon, S., Boesswetter, A., Bagdonat, T., Motschmann, U., 2007a, Physics of the Ion Composition Boundary: a comparative 3-D hybrid simulation study of Mars and Titan, Annales Geophysicae, 25, 99-115

Simon, S., Boesswetter, A., Bagdonat, T., Motschmann, U., Schuele, J., 2007b, Threedimensional multispecies hybrid simulation of Titan's highly variable plasma environment, Annales Geophysicae, 25, 117-144

Sonett, C. P., Coleman, P. J., Wilcox, J. M. (Eds.), 1972, Solar Wind, National Aeronautics and Space Administration, NASA SP-308

Tian, F., Toon, O. B., Pavlov, A. A., De Sterck, H., 2005, Transonic Hydrodynamic Escape of Hydrogen from Extrasolar Planetary Atmospheres, Astrophysical Journal, 621, 1049-1060

Treumann, R. A., Jaroschek, C. H., 2008, Fundamentals of Non-relativistic Collisionless Shock Physics: I. The Shock Problem, ArXiv e-prints, arXiv:0805.2132

Vidal-Madjar, A., Lecavelier des Etangs, A., Désert, J.-M., Ballester, G. E., Ferlet, R., Hébrard, G., Mayor, M., 2003, An extended upper atmosphere around the extrasolar planet HD209458b, Nature, 422, 143-146

Vidal-Madjar, A., Désert, J.-M., Lecavelier des Etangs, A., Hébrard, G., Ballester, G. E., Ehrenreich, D., Ferlet, R., McConnell, J. C., Mayor, M., Parkinson, C. D., 2004, Detection of Oxygen and Carbon in the Hydrodynamically Escaping Atmosphere of 
the Extrasolar Planet HD 209458b, Astrophysical Journal, Letters, 604, L69-L72, arXiv:astro-ph/0401457

Voigt, G.-H., 1995, Magnetospheric configuration, pp. 333-388, CRC Press

Watson, A. J., Donahue, T. M., Walker, J. C. G., 1981, The dynamics of a rapidly escaping atmosphere - Applications to the evolution of earth and Venus, Icarus, 48, 150-166

Weber, E. J., Davis, L. J., 1967, The Angular Momentum of the Solar Wind, Astrophysical Journal, 148, 217-227

Wolszczan, A., Frail, D. A., 1992, A planetary system around the millisecond pulsar PSR1257 + 12, Nature, 355, 145-147

Yelle, R. V., 2004, Aeronomy of extra-solar giant planets at small orbital distances, Icarus, $170,167-179$

Zarka, P., 1998, Auroral radio emissions at the outer planets: Observations and theories, Journal of Geophysics Research, 103, 20 159-20 194

Zarka, P., Treumann, R. A., Ryabov, B. P., Ryabov, V. B., 2001, Magnetically-Driven Planetary Radio Emissions and Application to Extrasolar Planets, Astrophysics and Space Science, 277, 293-300 


\section{Publications}

\section{Refereed publications}

Johansson, E. P. G., Bagdonat, T., \& Motschmann, U., 2009, "Consequences of expanding exoplanetary atmospheres for magnetospheres”, A\&A, 496, 869

\section{Oral presentations and posters}

Johansson, E. P. G., Bagdonat, T., \& Motschmann, U., "Stellar wind interaction with close-in extrasolar planets", JENAM/MIST/UKSP 20-23 April 2009, Hatfield (Hertfordshire), United Kingdom (Oral)

Johansson, E. P. G., Bagdonat, T., \& Motschmann, U., "Hybrid simulations of the stellar wind interaction with close-in extrasolar planets", Planet Formation and Evolution: The Solar System and Extrasolar Planets, 2-6 March 2009, Tübingen (Poster)

Johansson, E. P. G., Bagdonat, T., \& Motschmann, U., "Hybrid simulations of the stellar wind interaction with close-in extrasolar planets", International Max-Planck Research School on Physical Processes in the Solar System and Beyond, Max Planck Institute for Solar System Research, Katlenburg-Lindau, 11 February 2009 (Oral)

Johansson, E. P. G., Bagdonat, T., \& Motschmann, U., "Consequences of expanding atmospheres for magnetospheres", JENAM 8-12 September 2008, Vienna, Austria (Oral)

Johansson, E. P. G., Bagdonat, T., \& Motschmann, U., "Hybrid simulations of stellar wind interaction with close-in extrasolar planets", Winter school on Physics and Astrophysics of Planetary Systems 18-29 February 2008, Les Houches, France (Oral)

Johansson, E. P. G., Bagdonat, T., \& Motschmann, U., "Close-in extrasolar planets and hybrid simulations of their interaction with the stellar wind", International Max-Planck Research School on Physical Processes in the Solar System and Beyond, Max Planck Institute for Solar System Research, Katlenburg-Lindau 13 February 2008 (Oral)

Johansson, E. P. G., Bagdonat, T., \& Motschmann, U., "Expanding atmospheres of close-in extrasolar planets and their interaction with the stellar wind", 5th Planet Formation Workshop, 19-21 September 2007, Braunschweig (Oral) 


\section{Other publications}

Johansson, E. P. G., Bagdonat, T., \& Motschmann, U., 2010, "Hybrid simulations of stellar wind interaction with close-in extrasolar planets", in "Physics and Astrophysics of Planetary Systems“", (Ed.) T. Montmerle, D. Ehrenreich, \& A.-M. Lagrange, vol. 41 of EAS Publications Series, pp. 459-462 


\section{Acknowledgments}

First of all I would like to thank my supervisor Prof. Dr. Uwe Motschmann for offering me the opportunity to work within the very, very interesting field of extrasolar planets and for his assistance, advice, support, insights into physics as well as valuable advice on oral presentations and finally for giving me the freedom to try my own ideas. I also of course would like to thank the International Max-Planck Research School (IMPRS) on "Physical Processes in the Solar System and Beyond" and its coordinator Dr. Dieter Schmitt at the Max Planck Institute for Solar System Research for granting me its scholarship which makes all of this possible, and for letting me be part of the school's activities and allowing me to take at least a little part in, and enjoy, the magnificent diversity of nationalities and languages among the IMPRS students.

I would also like to thank Joachim Müller for numerous discussions and insights into the physics of stellar wind interaction, the technicalities of the hybrid code and not the least for writing the new, better and more easy-to-understand version of it (AIKEF). Not to be understated is also his patience as a computer administrator with my sometimes very mysteriously failing work computers...

I thank Dr. Jean-Mathias Grießmeier for support and collaboration on extending the pressure balance equation and estimating standoff distances. I am also very grateful for several scientific discussions with both Dr. Alexander Bößwetter and Dr. Sven Simon. I am also thankful for some very time-saving advice on interpreting obscure numerical artefacts.

Moreover, I would like to thank many other in the work group and in its vicinity; Simon Großjohann (and Amy), Hendrik Kriegel, Christoph Koenders, Stefan Wiehle, Michael Dorn, Björn Willenberg, Ingo von Borstel, Dr. Gero Kleindienst, Frau Renate Strassek, Jinnee Yung-Ching Wang and others who have made the place a more fun and vivid place through all the occasional table football, table tennis, water skiing, volleyball, birthday cakes and more as well as by answering my many curious questions on German language, culture and miscellaneous oddities as well practicalities.

Last but not the least, I would like to thank my parents. Not always understanding what I am up to and why, but always supporting me anyway... 



\section{Curriculum Vitae}

\section{Persönliche Daten}

Name: $\quad$ Erik Per Gunnar Johansson

Geburtsdatum: $\quad$ 9. August, 1979

Geburtsort: Kungälv, Schweden

Staatsangehörigkeit: Schwedisch

\section{Schulausbildung}

1986-1992: Låg- och mellanstadieskola, Fontinskolan, Kungälv, Schweden

1992-1995: Högstadieskola, Thorildskolan, Kungälv, Schweden

1995-1998: Abitur, Ängelholms gymnasieskola, Ängelholm, Schweden

\section{Studium}

1999-2002, 2003-2005: Master (inkl. Bachelor) of Science in Engineering Physics ("Civilingenjör i teknisk fysik")

Technische Hochschule Chalmers, Göteborg, Schweden

2002-2003: $\quad$ Student der Universität Göteborg, Göteborg, Schweden in Psychologie und Praktische Philosophie

2007-2010: $\quad$ Doktorand am Institut für Theoretische Physik, TU Braunschweig mit einem Stipendium der "International Max Planck Research School on Physical Processes in the Solar System and Beyond" am Max Planck Institut für Sonnensystemforschung, Katlenburg-Lindau, Deutschland Betreuer: Prof. Dr. U. Motschmann

\section{Sonstiges}

1998-1999: Militärdienst (Wehrpflicht), Telegrafist, Schweden

2005-2006: Lehrer-Tätigkeit, Mathematik und Programmierung Technische Hochschule Chalmers, Göteborg, Schweden 